RECEIVED

FEB $2^{9} 1996$

ORNLTM-13042

\section{Advanced Neutron Source Dynamic Model (ANSDM) Code Description and User Guide}

Jose March-Leuba

August 1995

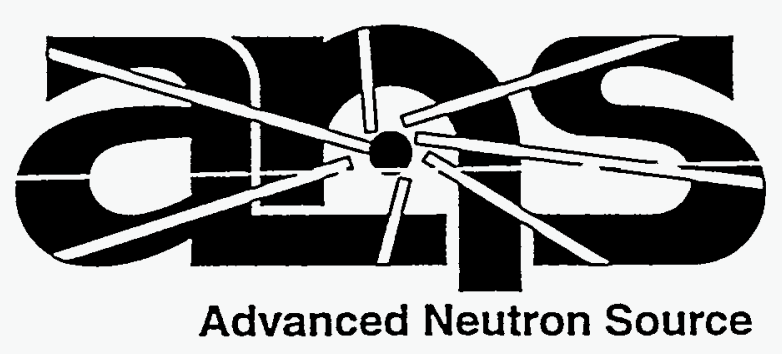

MANAGED BY

MARTIN MARIETTA ENERGY SYSTEMS, INC.

FOR THE UNITED STATES

DEPARTMENT OF ENERGY
TISTIIBUTION OF THIS DOCUMENT IS UNLIMITED swe

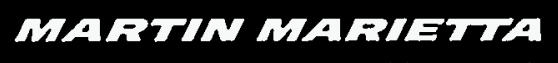


This report has been reproduced directly from the best available copy.

Available to DOE and DOE contractors from the Office of Scientific and Technical Information, P.O. Box 62, Oak Ridge, TN 37831; prices available from (615) 576-8401, FTS 626-8401.

Available to the public from the National Technical Information Service, U.S. Department of Commerce, 5285 Port Royal Rd., Springfield, VA 22161.

This report was prepared as an account of work sponsored by an agency of the United States Government. Neither the United States Government nor any agency thereof, nor any of their employees, makes any warranty, express or implied, or assumes any legal liability or responsibility for the accuracy, completeness, or usefulness of any information, apparatus, product, or process disclosed, or represents that its use would not infringe privately owned rights. Reference herein to any specific commercial product, process, or service by trade name, trademark, manufacturer, or otherwise, does not necessarily constitute or imply its endorsement, recommendation, or favoring by the United States Government or any agency thereof. The views and opinions of authors expressed herein do not necessarily state or reflect those of the United States Government or any agency thereot. 


\author{
Jose March-Leuba
}

Date published: August 1995

Prepared by

OAK RIDGE NATIONAL LABORATORY

Oak Ridge, Tennessee 37831

managed by

LOCKHEED MARTIN ENERGY SYSTEMS

for the

U.S. DEPARTMENT OF ENERGY

under contract DE-AC05-84OR21400

DISTPIBUTION OF THIS DOCUMENT IS UNLIMTED 

LIST OF FIGURES $\ldots \ldots \ldots \ldots \ldots \ldots \ldots \ldots \ldots \ldots \ldots \ldots \ldots \ldots \ldots \ldots \ldots \ldots \ldots \ldots$

LIST OF TABLES $\ldots \ldots \ldots \ldots \ldots \ldots \ldots \ldots \ldots \ldots \ldots \ldots \ldots \ldots \ldots \ldots \ldots \ldots \ldots \ldots \ldots \ldots$

ACRONYMS AND INITIALISMS $\ldots \ldots \ldots \ldots \ldots \ldots \ldots \ldots \ldots \ldots \ldots \ldots \ldots \ldots \ldots \ldots \ldots \ldots \ldots$

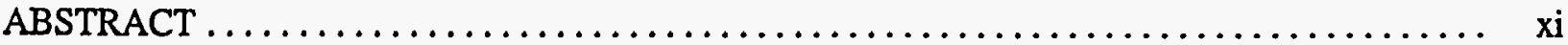

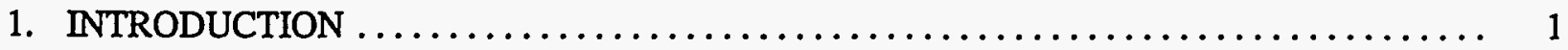

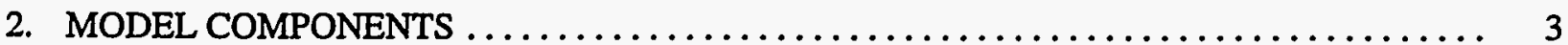

2.1 CORE AND CORE PRESSURE BOUNDARY TUBE REGIONS $\ldots \ldots \ldots \ldots \ldots \ldots \ldots$.

2.2 REFLECTOR REGION $\ldots \ldots \ldots \ldots \ldots \ldots \ldots \ldots \ldots \ldots \ldots \ldots \ldots \ldots \ldots \ldots \ldots$

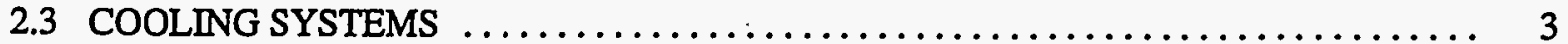

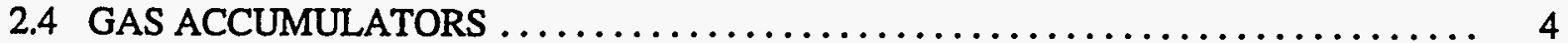

2.5 MAKEUP AND LETDOWN SYSTEMS $\ldots \ldots \ldots \ldots \ldots \ldots \ldots \ldots \ldots \ldots \ldots \ldots \ldots \ldots \ldots$

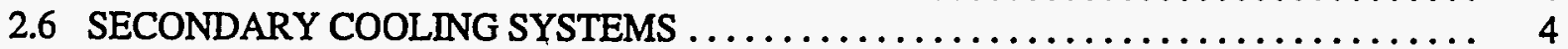

2.7 CONTROL SYSTEMS $\ldots \ldots \ldots \ldots \ldots \ldots \ldots \ldots \ldots \ldots \ldots \ldots \ldots \ldots \ldots \ldots \ldots \ldots \ldots$

2.8 INSTRUMENTATION $\ldots \ldots \ldots \ldots \ldots \ldots \ldots \ldots \ldots \ldots \ldots \ldots \ldots \ldots \ldots \ldots \ldots \ldots \ldots$

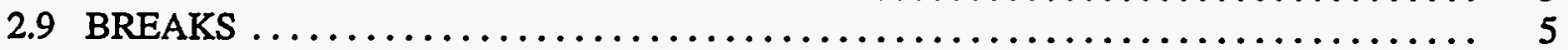

3. MODELS AND EMPIRICAL CORRELATIONS $\ldots \ldots \ldots \ldots \ldots \ldots \ldots \ldots \ldots \ldots \ldots \ldots \ldots$

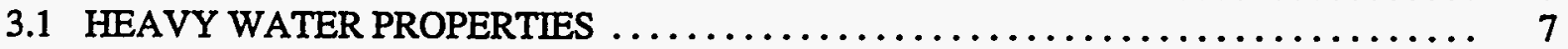

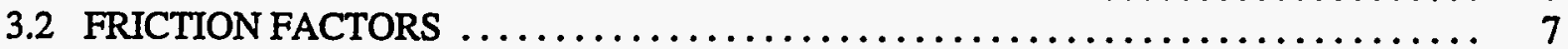

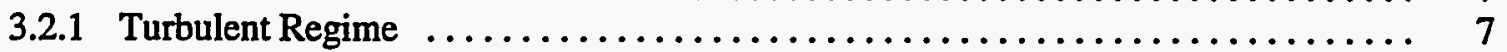

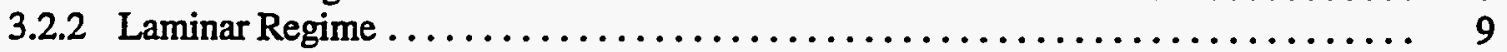

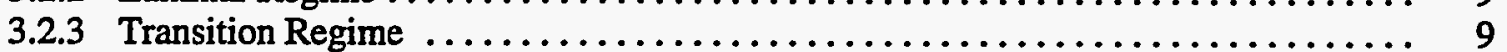

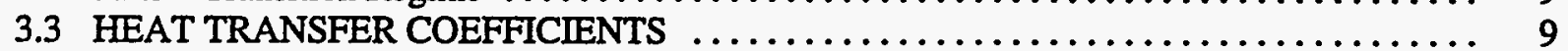

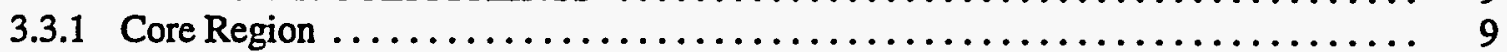

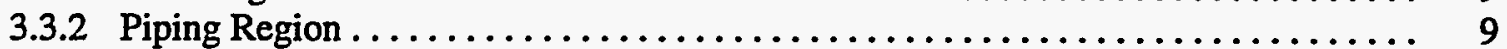

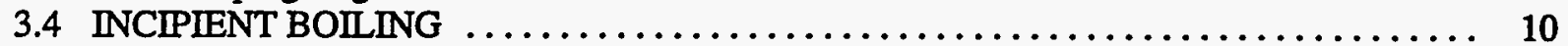

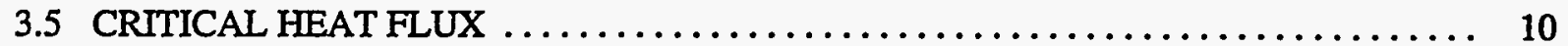

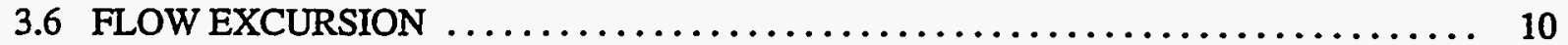

4. MODEL PARAMETERS $\ldots \ldots \ldots \ldots \ldots \ldots \ldots \ldots \ldots \ldots \ldots \ldots \ldots \ldots \ldots \ldots \ldots \ldots \ldots$

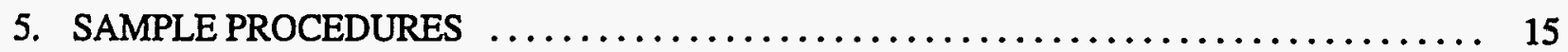

Appendix A. HEAVY WATER CORRELATIONS $\ldots \ldots \ldots \ldots \ldots \ldots \ldots \ldots \ldots \ldots \ldots \ldots \ldots \ldots$

Appendix B. COOLANT-RELATED CORRELATIONS $\ldots \ldots \ldots \ldots \ldots \ldots \ldots \ldots \ldots \ldots \ldots$ B-1

Appendix C. HEAT TRANSFER AND CRITICAL HEAT FLUX CORRELATIONS $\ldots \ldots \ldots \ldots$ C-1

Appendix D. STANDARD MACRO DEFINITION FILE (ANS.CMD) FOR ANSDM $\ldots \ldots \ldots \ldots$ D-1 Appendix E. NOMINAL ANS OPERATING CONDITIONS PREDICTED BY ANSDM ...... E-1 Appendix F. LIST OF ALL ANSDM MODEL VARIABLES AND THEIR

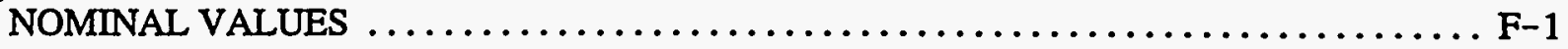





\section{LIST OF FIGURES}

Figure

Page

1 Friction factor calculated by Advanced Neutron Source Dynamic Model after scram and transition to natural circulation. 
. 


\section{LIST OF TABLES}

\section{Table}

Page

1 Interpretation of the first two characters of Advanced Neutron Source Dynamic

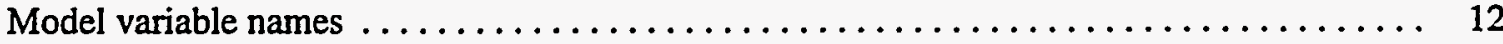

2 Interpretation of the node characters in Advanced Neutron Source Dynamic Model

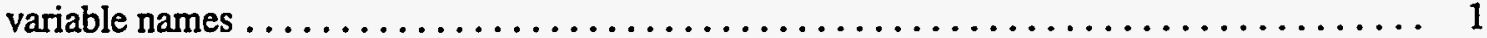

3 Interpretation of the node interface characters in Advanced Neutron Source Dynamic Model variable names 



\section{ACRONYMS AND INITIALISMS}

ACSL Advanced Continuous Simulation Language

ANS Advanced Neutron Source

ANSDM Advanced Neutron Source Dynamic Model

$\mathrm{BOC}$ beginning of cycle

CHF critical heat flux

EOC end of cycle

LOCA loss-of-coolant accident 



\begin{abstract}
A mathematical model is designed that simulates the dynamic behavior of the Advanced Neutron Source (ANS) reactor. Its main objective is to model important characteristics of the ANS systems as they are being designed, updated, and employed; its primary design goal, to aid in the development of safety and control features. During the simulations the model is also found to aid in making design decisions for thermal-hydraulic systems. Model components, empirical correlations, and model parameters are discussed; sample procedures are also given. Modifications are cited, and significant development and application efforts are noted focusing on examination of instrumentation required during and after accidents to ensure adequate monitoring during transient conditions.
\end{abstract}


. 


\section{INTRODUCTION}

The Advanced Neutron Source Dynamic Model (ANSDM) is a mathematical model that simulates the dynamic behavior of the Advanced Neutron Source (ANS) reactor in a personal computer. The main objective was to model important characteristics of the ANS systems as they were being designed, updated, and employed. The primary ANSDM design goal was to aid the development of safety and control features, but it also has aided in making design decisions for thermal-hydraulic systems. The model has been modified to evaluate possible design changes, to study the performance of control algorithms, and to support safety analyses. Significant ANSDM development and application efforts have been focused on examining the instrumentation required during and after accidents to ensure adequate monitoring during transient conditions.

The most important limitations of this model are:

- Point kinetics for the neutron dynamics in the core region. The power is distributed among different components (i.e, upper and lower fuel elements, reflector, bypass region) based on steady-state power fraction distributions that have been estimated for the specific ANS conditions. This is not such a bad approximation because most transients result in a reactor scram within the first few milliseconds and then the power is determined by a decay heat correlation.

- Incompressible flow. The model is limited to liquid-phase state; when a transient results in saturated boiling, the simulation fails. Note that the core typically is damaged (owing either to critical heat flux or to flow excursion instabilities) well before saturated boiling can be established; thus, this approximation is fairly accurate except when acoustic wave propagation is a relevant effect such as during large-break loss-of-coolant accidents (LOCAs).

- Single loop flow dynamics. All three active loops are simulated by one effective loop. Because of this approximation, the model is not able to simulate imbalances between loops (e.g., we cannot model directly the shutdown of one pump while the other two remain on). These imbalances must be simulated by, for example, reducing the pump speed to $80 \%$ to simulate a one-out-of-three pump trip.

- Poor reverse flow model. The model fails to compute enthalpies properly if reverse flow is established. Note, however, that reverse flow occurs only in the hot leg during large-break LOCAs, and the hot leg enthalpy is fairly irrelevant during these transients. The reverse flow model in ANSDM is designed to "ride through" short (i.e., a few milliseconds) flow reversals that may occur during severe transients, which do not affect significantly the later evolution of the event. 



\section{MODEL COMPONENTS}

The ANSDM has been programmed in the Advanced Continuous Simulation Language, giving it fairly good flexibility of operation at run time. This model was designed for testing and defining control and plant protection system design requirements; it also has been used to evaluate reactivity events in the Conceptual Safety Analysis Report and other transient events to evaluate different design options. The model is composed of a collection of modules, most of which are reused throughout the model.

\subsection{CORE AND CORE PRESSURE BOUNDARY TUBE REGIONS}

The power generation in the core is estimated from the addition of two components: (1) the core neutronics (modeled using point kinetics) with delayed neutrons (including photo-delayed neutrons) and (2) decay heat (based on an ANS-specific correlation).

ANSDM models the average channel fuel and coolant dynamics. The average channel determines the average core outlet conditions. A single axial node is used for this calculation. The dynamics of the hot streak of the upper and lower element are simulated separately from the average channel calculation. The lower element is typically limited at beginning of cycle (BOC); the upper element, at end of cycle (EOC). Thus, in our model we use the BOC axial power shape and hot streak factors for the lower element hot channel and the EOC conditions for the upper element. The two hot channels are divided into up to 50 axial nodes (typically, we use 27) where local temperatures, pressures, and heat fluxes are estimated to determine its margin to incipient boiling, critical heat flux, and flow excursion instability.

A single bypass region simulates the flow of heavy water that bypasses the fuel elements inside the pressure vessel. This coolant is typically colder than the core outlet coolant such that when it mixes, the vessel outlet temperature (which is computed dynamically) is lower than the core outlet temperature.

\subsection{REFLECTOR REGION}

A reflector region is modeled with a very simplified one-node approach. The reflector provides some (but not much) reactivity feedback to the core owing to the direct neutron and gamma heating. ANSDM does not model the reflector coolant systems. The main purpose of this node is to provide for an estimate of the reflector temperature feedback.

\subsection{COOLING SYSTEMS}

Cooling system pipes are modeled, and they release heat to the appropriate surrounding light water pools. Whenever parallel flows exists (i.e., multiple hot legs), ANSDM uses a single pipe with an effective flow area and equivalent diameter.

Containment light water pools (i.e., the main reactor pool, the pipe chase pool, the heat exchangers pool) are modeled. These pools take heat from the reactor piping according to their relative temperature and based on natural convection heat transfer coefficients. The heat exchanger pool also cools the emergency heat exchanger secondary side by natural circulation.

The main heat exchanger is modeled with the primary flow in the shell side and with the secondary flow in the tube side. Heat transfer characteristics are adjustable by varying the tube diameter and surface area; typically, values include a fouling heat transfer resistance factor. 
The emergency heat exchanger is modeled in series with the main heat exchanger. Primary flow is in the shell side, and secondary flow is on the tube side. The shell side (primary) assumes "turbulizers" so that the flow is never laminar regardless of Reynolds number. The tube's diameter is designed to be about $0.05 \mathrm{~m}$ ( 2 in.) so that the Reynolds number will be large enough to ensure turbulent flow even at the low natural circulation flow rates. The secondary side of the emergency heat exchanger is connected to the heat exchanger pool and allowed to flow by natural circulation.

Main circulation pumps are modeled according to the head-flow characteristic curve. The characteristic curve scales the flow directly proportional to the pump rotational speed; the pump head is proportional to the square of the pump speed; and the power required is proportional to the cube of the speed. Pump coast-down is modeled based on a conservation of angular momentum; the resulting differential equation that is solved by the model is:

$$
\frac{d n}{d t}=\frac{n^{2}-n_{0}^{2}}{\tau},
$$

where $n$ is the pump rotational speed, $n_{0}$ is the desired equilibrium speed (e.g., $n_{0}=10 \%$ if a reduction to pony flow is desired), and $\tau$ is the pump half speed time constant. The coast-down flow and pump head are computed by scaling the characteristic pump curve using the calculated speed, $n$.

\subsection{GAS ACCUMULATORS}

The gas accumulator is assumed to follow the ideal gas law $\left(P V^{\gamma}=\right.$ constant). The default values in ANSDM assume that the accumulators expand isothermally (i.e., $\gamma=1.0$ ), but a model parameter (KGCGAC) can be changed to 1.4 for an adiabatic expansion. The initial gas-to-liquid ratio is such that the liquid level will not reach the bottom of the accumulator after the gas has expanded to the depressurized condition; for a 2.0-MPa core outlet pressure, the liquid-to-gas ratio is 20 to 1 .

\subsection{MAKEUP AND LETDOWN SYSTEMS}

The reactor pressure is maintained high by a makeup flow. The model simulates this flow with a pump module (i.e., the pressurizer pump) with a suction in a constant pressure tank (i.e., the letdown system tank). The makeup pump speed is maintained constant unless a coast-down (e.g., loss of off-site power) is required. During normal operation the makeup flow adjusts itself to the system pressure (e.g., as the system pressure lowers, the makeup flow increases). These changes, however, are not sufficient to maintain constant pressure. The pressure regulation is accomplished by modulating the flow through the letdown valves. The letdown valves are modeled as a pressure drop with variable coefficient (according to valve opening); the letdown flow is collected in the letdown tank. The model does not simulate the low-pressure cleanup system, and this tank is assumed to have an infinite supply of $\mathrm{D}_{2} \mathrm{O}$ such that makeup can always be maintained. Makeup supply problems can be simulated at any time by tripping the makeup pump that is simulated with a perfect (i.e, no reverse flow) check valve.

\subsection{SECONDARY COOLING SYSTEMS}

The secondary side of the ANS cooling system is represented by:

- the secondary side of the main heat exchanger in the tube side, 
- the secondary hot leg,

- main cooling towers and the cooling towers basin,

- the secondary circulation pump, and

- the secondary cold leg.

All these components use approximations similar to those in the primary system.

\subsection{CONTROL SYSTEMS}

A preliminary control system, simulated in the model, includes:

- control rod position based on the measured power-to-flow ratio,

- pressure control that actuates the letdown valve based on hot-leg pressure measurements, and

- core inlet temperature control that actuates on the secondary flow based on the temperature measured at the heat exchanger outlet.

\subsection{INSTRUMENTATION}

Sensor dynamics are modeled as first-order lag systems. The required time constants have been determined through simulation of control and plant protection system challenges. The time constants currently in the model are those required to satisfy most design basis events requirements.

\subsection{BREAKS}

Breaks are simulated as a flow through an orifice (of the break effective diameter) from the inside of the main piping system to the light water pools. The leak flow, $W_{l k}$, is estimated from the orifice relation as

$$
W_{l k}=\frac{C_{v}}{\sqrt{1-\left(\frac{D_{b}}{D}\right)^{4}}} \frac{\pi D^{2}}{4} \sqrt{2 \rho\left(P-P_{l k}\right)},
$$

where $P_{l k}$ is the pool pressure, $P$ is the system pressure, $C_{v}$ is the orifice coefficient (taken as 0.6 for a sharp orifice), $D_{b}$ is the break effective diameter, and $D$ is the pipe diameter.

Breaks are opened exponentially over a finite period of time, $\tau$, following the expression

$$
D(t)=D_{b}\left(e^{\frac{t}{\tau} \ln 2}-1\right) \text { if } 0 \leq t \leq \tau,
$$

and

$$
D(t)=D_{b} \quad \text { if } t \geq \tau,
$$

where $D(t)$ is the effective break diameter, $D_{b}$ is the final break size, and $\tau$ is the break time constant (typically $250 \mathrm{~ms}$ ). ANSDM does not model the compressibility of $\mathrm{D}_{2} \mathrm{O}$ such that the effective speed of sound is infinite. Therefore, ANSDM cannot model fast-opening large breaks where the pressure perturbations are large and speed-of-sound effects are relevant. 



\section{MODELS AND EMPIRICAL CORRELATIONS}

This section documents the empirical correlations used in ANSDM. Unless otherwise stated, the units for all correlations are SI, energy is in joules, temperature is in degrees Celsius, length is in meters, and time is in seconds.

\subsection{HEAVY WATER PROPERTIES}

ANSDM uses the standard ANS $\mathrm{D}_{2} \mathrm{O}$ correlations that define some $\mathrm{D}_{2} \mathrm{O}$ properties required by ANSDM as a function of known parameters. Appendix A documents the specific correlation parameters and units used in ANSDM; the actual FORTRAN Program used is shown.

\subsection{FRICTION FACTORS}

Appendix B lists the actual FORTRAN coding used to estimate the friction factor. Figure 1 shows the friction factor calculated by ANSDM following a scram and complete loss of pumping power (i.e., natural circulation). This section discusses some of the approximations used.

\subsubsection{Turbulent Regime}

In the turbulent regime ( $R e>4240$ ), ANSDM uses two different correlations to estimate the Darcy friction factor: the Filonenko correlation in the core region and the Colebrook and White correlation for the piping sections. If a relative roughness other than zero is input, ANSDM uses the Colebrook and White correlation; otherwise, it uses the Filonenko correlation.

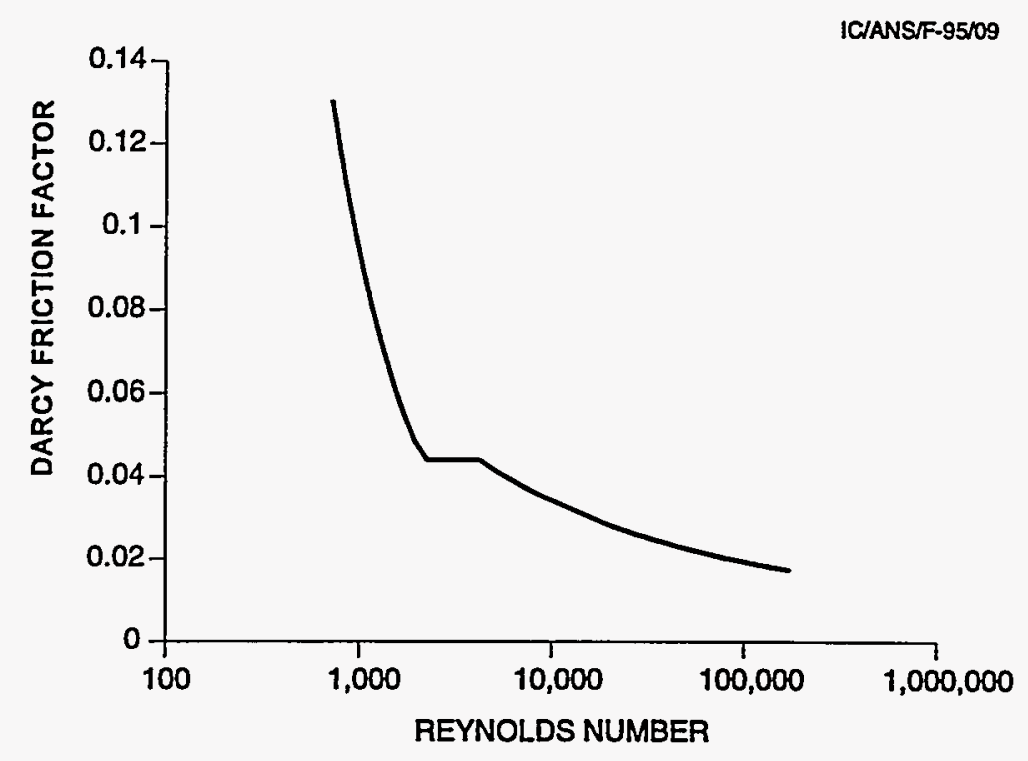

Fig. 1. Friction factor calculated by the Advanced Neutron Source Dynamic Model after scram and transition to natural circulation. 
The Filonenko correlation used for ANS design applies to smooth surfaces. When a correction for rectangular channels and coolant properties is included, the correlation takes the form.

$$
f_{D}=\frac{1.0875-0.1125(b / s)}{\left(1.82 \log _{10} R e_{b}-1.64\right)^{2}}\left[\frac{7-\left(\mu_{b} / \mu_{w}\right)}{6}\right]
$$

ANSDM does not use this form of the correlation. It uses the following approximation:

$$
f_{D}=\frac{1.0836}{\left(1.82 \log _{10} R e_{b}-1.64\right)^{2}}
$$

ANSDM collapses both the upper and lower fuel elements into a single average channel; thus, it uses an average of the span-to-gap aspect ratio $(b / s)$ for the upper and lower elements, resulting in the term 1.8036 , which includes an average aspect ratio for both cores.

The last term in Eq. (5) is a correction proposed by Petukhov to account for the effect of heated fluids, which have lower viscosity near the wall. ANSDM ignores this term to avoid the need for iterative solutions that involve the fuel temperature. The effect of this term is fairly small. For instance, the core average coolant outlet temperature is $85^{\circ} \mathrm{C}$, and the average fuel wall temperature is $121^{\circ} \mathrm{C}$. With these numbers, the error introduced by neglecting the second term in Filonenko's correlation is about $7 \%$ (ANSDM estimates higher friction than the actual one). This error is partly offset by the fact that ANSDM estimates the pressure drop based on the outlet conditions of a single axial node in the core region. We estimate the error caused by this single-node approximation to be about $10 \%$ (the friction factor at $85^{\circ} \mathrm{C}$ is 0.0174 and at $45^{\circ} \mathrm{C}$ is 0.0209 ; the average is 0.0191 , or about $10 \%$ higher than ANSDM estimates). Thus, ANSDM assumes that the 7\% error introduced by ignoring the second term in Filonenko's correlation is partially offset by the $10 \%$ error caused by the single-node approximation. The error caused by these two approximations diminishes as the power is decreased (e.g., following scram). Because of these approximations, the pressure-drop estimate accuracy in ANSDM is assumed to be $\pm 10 \%$ but is probably lower owing to cancellation of errors.

In the piping region the Filonenko correlation is not applicable because the pipes have an appreciable roughness factor, $\epsilon$. In this region ANSDM uses the Colebrook and White correlation. The friction factor is estimated by direct iteration from the following implicit equation:

$$
\sqrt{f_{D} / 4}=\frac{1}{3.48-1.7372 \ln \left(\frac{\epsilon}{2 D_{e}}+\frac{9.35}{R e \sqrt{f_{D} / 4}}\right)} .
$$

To initiate the iteration, ANSDM uses the Moody correlation as the initial guess:

$$
f_{D} / 4=0.001375\left[1+21.544\left(\frac{\epsilon}{2 D_{e}}+\frac{100}{R_{e}}\right)^{\frac{1}{3}}\right] .
$$




\subsubsection{Laminar Regime}

In the laminar regime ANSDM uses the standard laminar friction factor correlation:

$$
f_{D}=4 \frac{23.532}{R e}
$$

\subsubsection{Transition Regime}

To avoid possible numerical instabilities, the transition regime is modeled at Reynolds numbers lower than 4240 by selecting the higher of two numbers: the laminar friction factor at the actual Reynolds number or the turbulent friction factor at $R e=4240$. This approximation results in a constant friction factor for Reynolds numbers between 2300 and 4240 . In this way, a smooth transition between laminar and turbulent is achieved.

\subsection{HEAT TRANSFER COEFFICIENTS}

\subsubsection{Core Region}

Appendix $\mathrm{C}$ documents the actual FORTRAN coding for heat flux and heat transfer correlations. In the fully developed turbulent regime $(R e>2300)$ ANSDM uses the Petukhov correlation to estimate the heat transfer between the fuel plates and the core coolant. This correlation takes the form

$$
h_{\text {turbulent }}=\frac{k}{D_{e}} \frac{\left(f_{D} / 8\right) \operatorname{Re}_{b} \operatorname{Pr}_{b}\left(\mu_{b} / \mu_{w}\right)^{0.11}}{\left(1+3.4 f_{D}\right)+\left(11.7+\frac{1.8}{\operatorname{Pr}^{1 / 3}}\right) \sqrt{f_{D} / 8}\left(\operatorname{Pr}^{2 / 3}-1\right)} .
$$

In the laminar regime $(R e<2300)$ ANSDM uses a constant Nusselt number of 7.627 with a correction for heated fluid. The actual correlation takes the form

$$
h_{\mathrm{laminar}}=\frac{k}{D_{e}} 7.627\left(\mu_{b} / \mu_{w}\right)^{0.11}
$$

\subsubsection{Piping Region}

ANSDM estimates the heat lost to the containment light-water pools. The heat transfer coefficient inside the circular pipes is estimated using the following correlations:

and

$$
\begin{gathered}
h_{\text {turbulent }}=\frac{k}{D_{e}}\left[6.3+\frac{0.079 \sqrt{f_{D}^{18}} \operatorname{Re} \operatorname{Pr}}{\left(1+P_{r}^{0.8}\right)^{5 / 6}}\right], \\
h_{\text {laminar }}=\frac{k}{D_{e}} 4.364 .
\end{gathered}
$$

The heat transfer coefficient outside of the pipes is input manually, and it is maintained constant during all transients. 


\subsection{INCIPIENT BOILING}

ANSDM uses an incipient boiling correlation based on local conditions at the hot spot. The correlation used is that of Bergles and Rosenhow. The incipient boiling flux is estimated by iteration between the two following equations:

$$
Q_{I B}=0.9 \times 1.7978 \times 10^{-6} \times P^{1.156} \times\left[1.8\left(T_{w}-T_{b}\right)\right]^{\left(2.8285 / P^{0.0234}\right)},
$$

and

$$
Q_{I B}=h\left(T_{w}-T_{b}\right)
$$

where $h$ is the local heat transfer coefficient estimated using the Petukhov correlation.

\subsection{CRITICAL HEAT FLUX}

ANSDM estimates the critical heat flux (CHF) using the Gambill additive CHF correlation, the Weatherhead correlation for CHF wall temperature, and the Petukhov heat transfer coefficient correlation. The flowing equations are used:

and

$$
Q_{\text {pool }}=0.18 h_{f g} \rho_{v}\left(\sigma g \frac{\rho_{l}-\rho_{v}}{\rho_{v}^{2}}\right)^{\frac{1}{4}}\left(1+\left[\frac{\rho_{l}}{\rho_{v}}\right]^{\frac{3}{4}} c_{p} \frac{T_{\text {sat }}-T_{\text {bulk }}}{9.8 h_{f g}}\right),
$$

$$
Q_{\text {crit }}=Q_{\text {pool }}+h\left(T_{\text {wall }}-T_{\text {bulk }}\right) \text {, }
$$

where $h$ is the local heat transfer coefficient estimated using the Petukhov correlation, and $T_{\text {wall }}$ (i.e., the critical wall temperature) is estimated from the following equation:

$$
T_{\text {wall }}=T_{\text {sat }}+\left(47.7-0.127 T_{\text {sat }}\right)\left(\frac{Q_{\text {crit }}}{3.1546 E 6}\right)^{\frac{1}{4}} \text {. }
$$

Because $T_{\text {wall }}$ depends on the critical flux, $Q_{\text {crit }}$, the solution of the above equation requires an iteration procedure. To initiate the iteration, $T_{\text {wall }}$ is estimated without the Weatherhead correction using the following equation:

$$
T_{\text {wall }}=T_{\text {sat }}+84.96-0.1313\left(T_{\text {sat }}+273.16\right)
$$

\subsection{FLOW EXCURSION}

Flow excursion is a special case of critical heat flux that is caused by a flow instability rather than by a change in boiling regime. ANSDM uses the Costa correlation to estimate this critical heat flux:

$$
Q_{\text {crit }}=\frac{\left(T_{\text {sat }}-T_{\text {bulk }}\right) \sqrt{u}}{12.8 E-6}
$$




\section{MODEL PARAMETERS}

All model variables are accessible at run time for display, and they can also be modified at any time. In this way, different power levels or reactor configurations can be input at run time with simple "set" commands that can be prepared in a command file for multiple uses or that can be input from the keyboard. The variable nomenclature, thus, is important. This section describes this nomenclature, and Appendix $\mathrm{F}$ lists all variables and their nominal values.

An ACSL variable name has up to six characters. In ANSDM the first one, two, or three characters describe the type of parameter, and the last three or four characters describe the location on the model. For example, the variable $T C O R O$ represents the temperature (variable type $=T$ ) at the core outlet (location = CORO). Table 1 documents the interpretation of the first two characters, Table 2 documents the model nodes (i.e., three character locations that relate to node-average variables), and Table 3 documents the interconnection locations (i.e., four character locations that relate to node-boundary variables). 
Table 1. Interpretation of the first two characters of Advanced Neutron Source Dynamic Model variable names

\begin{tabular}{|c|c|c|c|c|c|}
\hline Characters & Variable type & Units & Characters & Variable type & Units \\
\hline CFR & $\begin{array}{l}\text { Critical heat flux ratio (Gambill } \\
\text { correlation) }\end{array}$ & Adimensional & $\mathbf{P}$ & Pressure & $\mathrm{Pa}$ \\
\hline CIR & Critical heat flux ratio (incipient boiling) & Adimensional & PS & Saturation pressure & $\mathrm{Pa}$ \\
\hline CSR & Critical heat flux ratio (flow excursion) & Adimensional & PR & Prandtl number & Adimensional \\
\hline CK & Coolant conductivity & $\mathrm{W} /(\mathbf{m} \cdot \mathbf{K})$ & $\mathbf{Q}$ & Heat flux & $\mathrm{W} / \mathrm{m}^{2}$ \\
\hline $\mathbf{C P}$ & Heat capacity & $\mathrm{J} /(\mathrm{kg} \cdot \mathrm{K})$ & $\mathbf{R}$ & Coolant density & $\mathrm{kg} / \mathrm{m}^{3}$ \\
\hline \multirow[t]{2}{*}{$\mathbf{F}$} & Neutron flux & $\mathrm{n} / \mathrm{m}^{2}$ & & or reactivity & Dollars \\
\hline & (also) friction coefficient & Adimensional & $\mathbf{R E}$ & Reynolds number & Adimensional \\
\hline $\mathbf{H}$ & Heat transfer coefficient & $W /\left(m^{2} \cdot K\right)$ & $\mathbf{T}$ & Temperature & $\mathrm{C}$ \\
\hline J & Power & $\mathbf{w}$ & TS & Saturation temperature & C \\
\hline $\mathbf{K}$ & $\begin{array}{l}\text { Constant parameter. Type depends on } \\
\text { next character }\end{array}$ & & $\mathbf{U}$ & Velocity & $\mathrm{m} / \mathrm{s}$ \\
\hline MU & Viscosity & $\mathrm{kg} /(\mathrm{s} \cdot \mathrm{m})$ & V & $\begin{array}{l}\text { Control variable. Type } \\
\text { depends on next character }\end{array}$ & \\
\hline MCR & $\begin{array}{l}\text { Minimum critical heat flux ratio along all } \\
\text { channels }\end{array}$ & Adimensional & W & Mass flow rate & $\mathrm{kg} / \mathrm{s}$ \\
\hline MIR & $\begin{array}{l}\text { Minimum incipient boiling ratio along } \\
\text { channel }\end{array}$ & Adimensional & $\mathbf{X}$ & Position & $\mathbf{m}$ \\
\hline MSR & $\begin{array}{l}\text { Minimum flow excursion ratio along all } \\
\text { channels }\end{array}$ & Adimensional & $\mathbf{Z}$ & $\begin{array}{l}\text { Internal node variable. Type } \\
\text { depends on next character }\end{array}$ & \\
\hline $\mathbf{N}$ & Angular speed (for pumps) & Normalized & . & & \\
\hline
\end{tabular}


Table 2. Interpretation of the node characters in Advanced Neutron Source Dynamic Model variable names

\begin{tabular}{|c|c|c|}
\hline Node & Module $^{a}$ & Description \\
\hline TRA & 1 & Externally imposed transients \\
\hline ANS & 1 & Main ANSDM module \\
\hline DET & 2 & Detectors \\
\hline $\mathrm{CON}$ & 2 & Controls \\
\hline PPS & 2 & Plant protection system \\
\hline SCRAM & 3 & Scram module \\
\hline ICR & 2 & Inner control rod \\
\hline OCR & 2 & Outer control rod \\
\hline VES & 2 & CPBT and core region \\
\hline REF & 3 & Reflector region \\
\hline IPR & 3 & Inlet plenum region \\
\hline COR & 3 & Core region \\
\hline CPG & 4 & Core power generation \\
\hline $\mathrm{CDH}$ & 5 & Core decay heat \\
\hline $\mathrm{CNT}$ & 5 & Core neutronics \\
\hline POI & 5 & Neutron poisons model \\
\hline ACC & 4 & Average channel coolant \\
\hline ACF & 4 & Average channel fuel \\
\hline $\mathrm{ACH}$ & 4 & Average channel \\
\hline BYP & 4 & Bypass \\
\hline $\mathrm{HCL}$ & 4 & Hot channel lower core \\
\hline $\mathrm{HCU}$ & 4 & Hot channel upper core \\
\hline HLC & 4 & Hot channel lower core coolant \\
\hline HLF & 4 & Hot channel lower core fuel \\
\hline HUC & 4 & Hot channel upper core coolant \\
\hline HUF & 4 & Hot channel upper core fuel \\
\hline OPR & 4 & Outlet plenum region \\
\hline CCS & 2 & Coolant cooling systems \\
\hline $\mathrm{CIL}$ & 3 & Core inlet leak \\
\hline CLR & 3 & Cold leg riser \\
\hline $\mathrm{COL}$ & 3 & Core outlet leak \\
\hline EHX & 3 & Emergency heat exchange \\
\hline EPP & 3 & Emergency heat exchanger primary side \\
\hline ESP & 3 & Emergency heat exchanger secondary \\
\hline GAC & 3 & Gas accumulator \\
\hline HLR & 3 & Hot leg riser \\
\hline $\mathrm{HXP}$ & 3 & Heat exchangers pool \\
\hline LDS & 3 & Letdown system \\
\hline MCL & 3 & Main cold leg (horizontal part) \\
\hline MCP & 3 & Main coolant pump \\
\hline MCS & 3 & Storage (controls pressure dynamics) \\
\hline MHL & 3 & Main hot leg (horizontal part) \\
\hline MHP & 3 & Main heat exchanger primary side \\
\hline MHX & 3 & Main heat exchange \\
\hline MRP & 3 & Main reactor pool \\
\hline MUS & 3 & Makeup system \\
\hline $\mathrm{PCH}$ & 3 & Pipe chase pool \\
\hline POL & 3 & Pump outlet leak module \\
\hline SHP & 3 & Main heat exchanger secondary side \\
\hline SCC & 3 & Secondary cooling circuits \\
\hline CTB & 4 & Cooling towers basin \\
\hline $\mathrm{TCL}$ & 4 & Secondary cold leg (tower to MHX) \\
\hline THL & 4 & Secondary hot leg (MHX to tower) \\
\hline
\end{tabular}

Numbers indicate parent module sequence. 
Table 3. Interpretation of the node interface characters in Advanced Neutron Source Dynamic Model variable names

\begin{tabular}{|c|c|}
\hline Node interface & Description \\
\hline ACHI & Average channel inlet \\
\hline ACHO & Average channel outlet \\
\hline AREA & Accident reactivity \\
\hline BYPI & Bypass inlet \\
\hline BYPO & Bypass outlet \\
\hline CILC & Core inlet leak at containment side \\
\hline CLRI & Cold leg riser inlet \\
\hline CLRO & Cold leg riser outlet \\
\hline CNFX & Core neutron flux \\
\hline COLC & Core outlet leak at containment side \\
\hline CORO & Core outlet at outlet plenum \\
\hline CROD & Control rods (inner + outer) \\
\hline CTBI & Cooling towers basin inlet (hot leg) \\
\hline CTBO & Cooling towers basin outlet (cold leg) \\
\hline DHFX & Core gamma flux \\
\hline DHFX & Decay heat fluxes \\
\hline ESCL & Secondary cold leg at emergency heat exchanger inlet \\
\hline ESHL & Secondary hot leg at emergency heat exchanger outlet \\
\hline HCLI & Hot channel lower core inlet \\
\hline HCLO & Hot channel lower core outlet \\
\hline HCUI & Hot channel upper core inlet \\
\hline HCUO & Hot channel upper core outlet \\
\hline HLRI & Hot leg riser inlet \\
\hline HLRO & Hot leg riser outlet \\
\hline HSFC & Hot spot coolant \\
\hline HXPI & Heat exchangers pool coolant flow inlet (cold) \\
\hline HXXPO & Heat exchangers pool coolant flow outlet (hot) \\
\hline $\mathrm{IPCI}$ & Inlet plenum outlet at core inlet \\
\hline LDSI & Letdown system inlet \\
\hline MCLI & Main cold leg inlet (at pump outlet leak location) \\
\hline MCLX & Emergency heat exchanger outlet (cold leg inlet) \\
\hline MCPI & Main coolant pump inlet \\
\hline MCPO & Main coolant pump outlet \\
\hline MCSO & Main cold leg at storage module outlet \\
\hline MHLA & Main hot leg at accumulator outlet \\
\hline MHLL & Main hot leg at letdown outlet \\
\hline MHLO & Main hot leg outlet \\
\hline MHXO & Main heat exchanger outlet \\
\hline MRPI & Main reactor pool coolant flow inlet (cold) \\
\hline MRPO & Main reactor pool coolant flow outlet (hot) \\
\hline MUSO & Makeup system outlet \\
\hline PCHI & Pipe chase pool coolant flow inlet (cold) \\
\hline PCHO & Pipe chase pool coolant flow outlet (hot) \\
\hline POLC & Pump outlet leak at containment side \\
\hline REFC & Reflector-core interface \\
\hline REFI & Reflector inlet \\
\hline REFO & Reflector outlet \\
\hline $\operatorname{SCLX}$ & Secondary cold leg at main heat exchanger inlet \\
\hline SHLX & Secondary hot leg at main heat exchanger outlet \\
\hline TCLI & Secondary cold leg inlet at tower \\
\hline TCLO & Secondary cold leg outlet at MHXX \\
\hline THLO & Secondary hot leg outlet at towers \\
\hline VESI & CPBT inlet \\
\hline VESO & CPBT outlet \\
\hline XESM & Neutron poisons \\
\hline
\end{tabular}




\section{SAMPLE PROCEDURES}

Within the ACSL the user can define macros to execute procedures in a consistent manner. Appendix $D$ shows the standard ANSDM macro file (ANS.CMD) that defines the most common procedures that can be exercised with ANSDM. In this we show and describe an example procedure to run a natural circulation transient.

Either at ACSL run time or by loading a command file, the user defines the following procedure, called natcir, that sets a transition to natural circulation by tripping the pump at the beginning of the transient. The procedure is defined as follows:

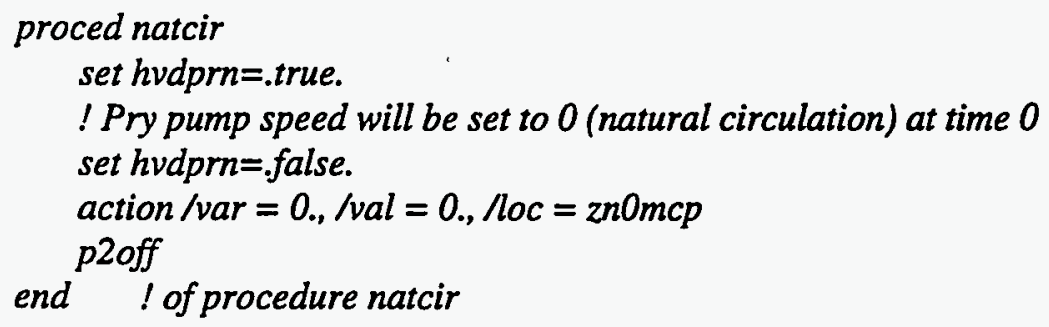

The first line defines the name of the procedure; this is further defined by the lines that follow and is delimited by the end statement. Comments following an exclamation mark (!) are ignored. Variable hvdprn is an internal ACSL variable that controls the high-volume display to the screen; it is originally set to .true. to output the comment to the screen and then set to false. to avoid unnecessary screen I/O. The action statement instructs ACSL to set variable $z n O m c p$ (i.e., the main coolant pump speed setpoint) to zero (/val $=0$.) at time zero $(/ v a r=0$.). The final statement $p 2$ off executes a procedure (or macro) that has been defined previously in file ANS.CMD (see Appendix D); this procedure trips the secondary cooling circuit pumps and resets the temperature control parameters.

To execute a transition to natural circulation, the user must invoke the natcir procedure and then start the simulation. In ACSL run time language, the following steps are performed:

\section{ACSL> natcir}

$A C S L>$ start

After the simulation is completed, the user may print or plot any of the variables that have been prepared (or saved). Appendix D lists the standard variables prepared automatically by ANS.CMD. Appendix E shows nominal operating conditions predicted by ANSDM. 
Appendix A

HEAVY WATER CORRELATIONS 
APPENDIX A. HEAVY WATER CORRELATIONS

c * Physical properties of D20

c * Offlcial ANS correlations as of September 30

1991

C * Memo from Moshw SIman-Tov to D.G. Selby

c * Notes some correlations here use different units

$c$ * than in the "official" correlations in the memo

c

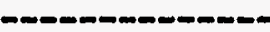

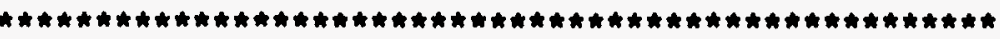

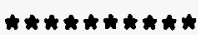

c

c D20 saturation Temperature

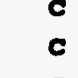$$
\text { c }
$$

c-

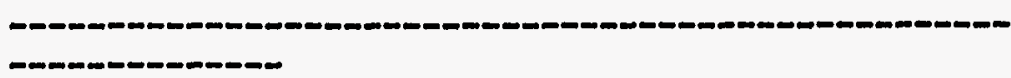

real function D_TSAT(P)

$\begin{array}{ll}\text { real } & P \\ \text { real } & a 0, a 1, a 2, a 3 \\ \text { data } & a 0 / 5.194927982 /\end{array}$

data
$a 1 / 2.36771673 e-1 /$, a0/5.194927982/,

$>\quad \mathrm{a} /-2.615268 \mathrm{e}-3 /, \mathrm{a} 3 / 1.708386 \mathrm{e}-3 /$
$x=\operatorname{alog}(A B S(P) / 1 . e 6)$

D_TSAT $=\exp (a 0+a 1 \star x+a 2 * x \star \star 2+a 3 \star x \star \star 3)$

rêturn end

c

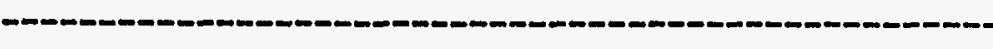

c

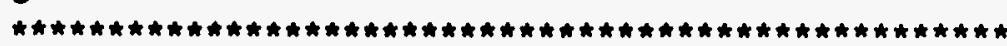

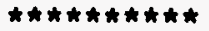

c

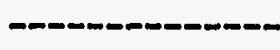

c D20 Liquid Density

c INPUT:

C T Temperature (C)

OUTPUT :

D_RHOL Liçuld density $(\mathrm{kg} / \mathrm{m} 3)$

$c$

$$
-
$$

$-\infty-\infty$

real function D_RHOL( $T$ )

real $T$

real a0, a1, a2

data a0/1.117772605e3/,

a1/-7.7855e-2/,

$.2 /-8.42 e-4 \mid$

$x=32 .+1.8 \star T$

D_RHOL $=20+a 1 * x+a 2 \star x * \star 2$

return

end 
c

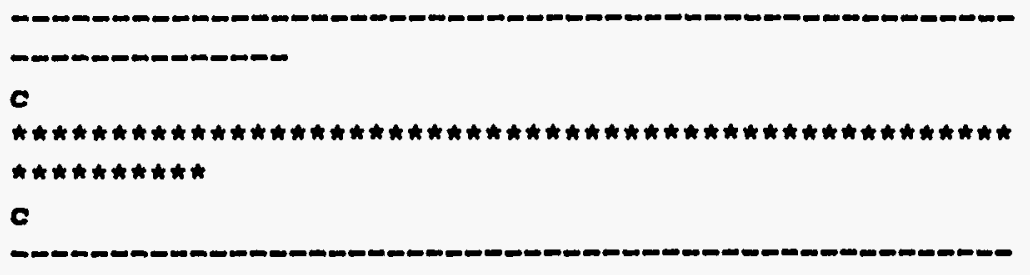

c D20 saturated Ilquid Thermal conductivity INPUT

OUTPUT

T Temperature (C)

D_K Liquid Conductivity $(\mathrm{H} / \mathrm{m} / \mathrm{K})$

c

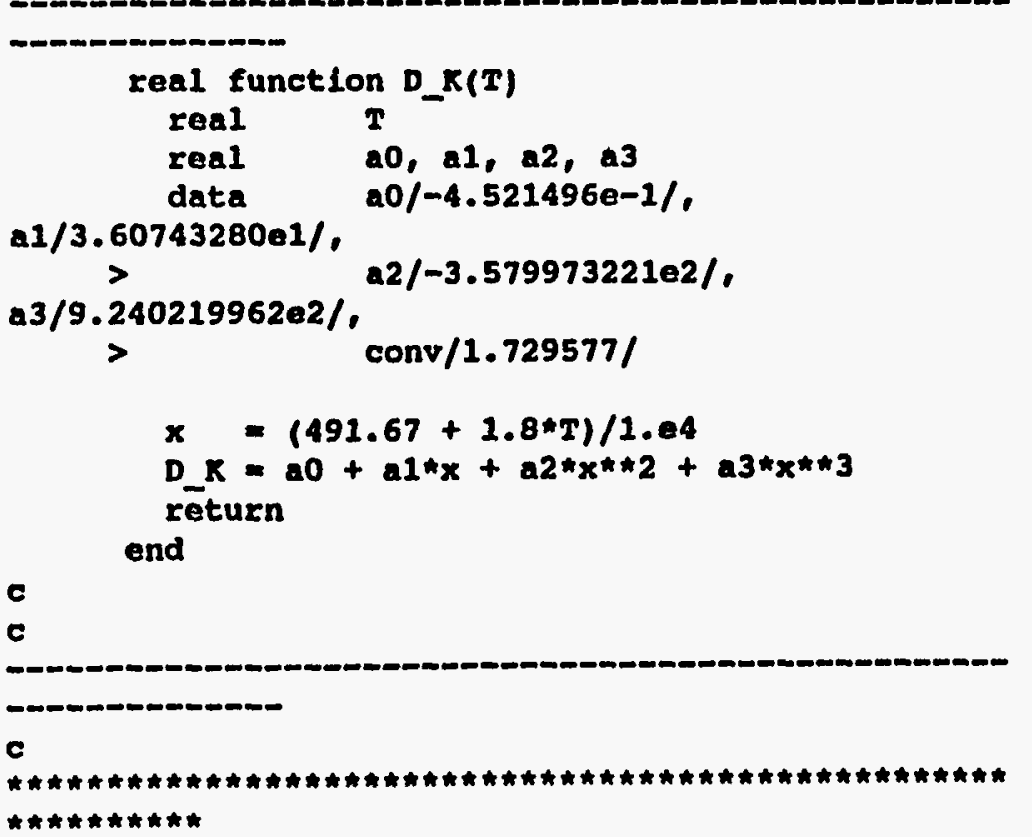

c

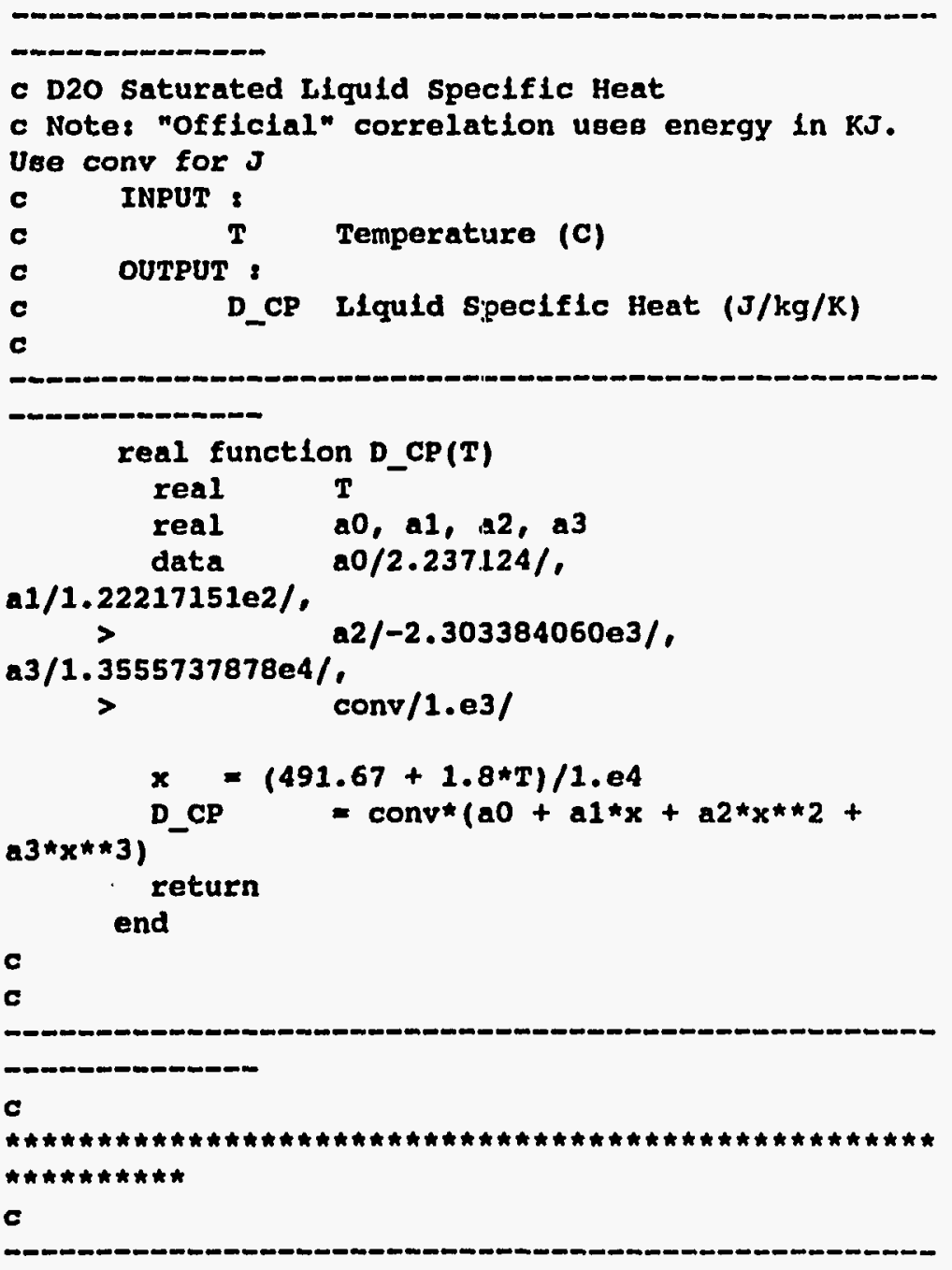




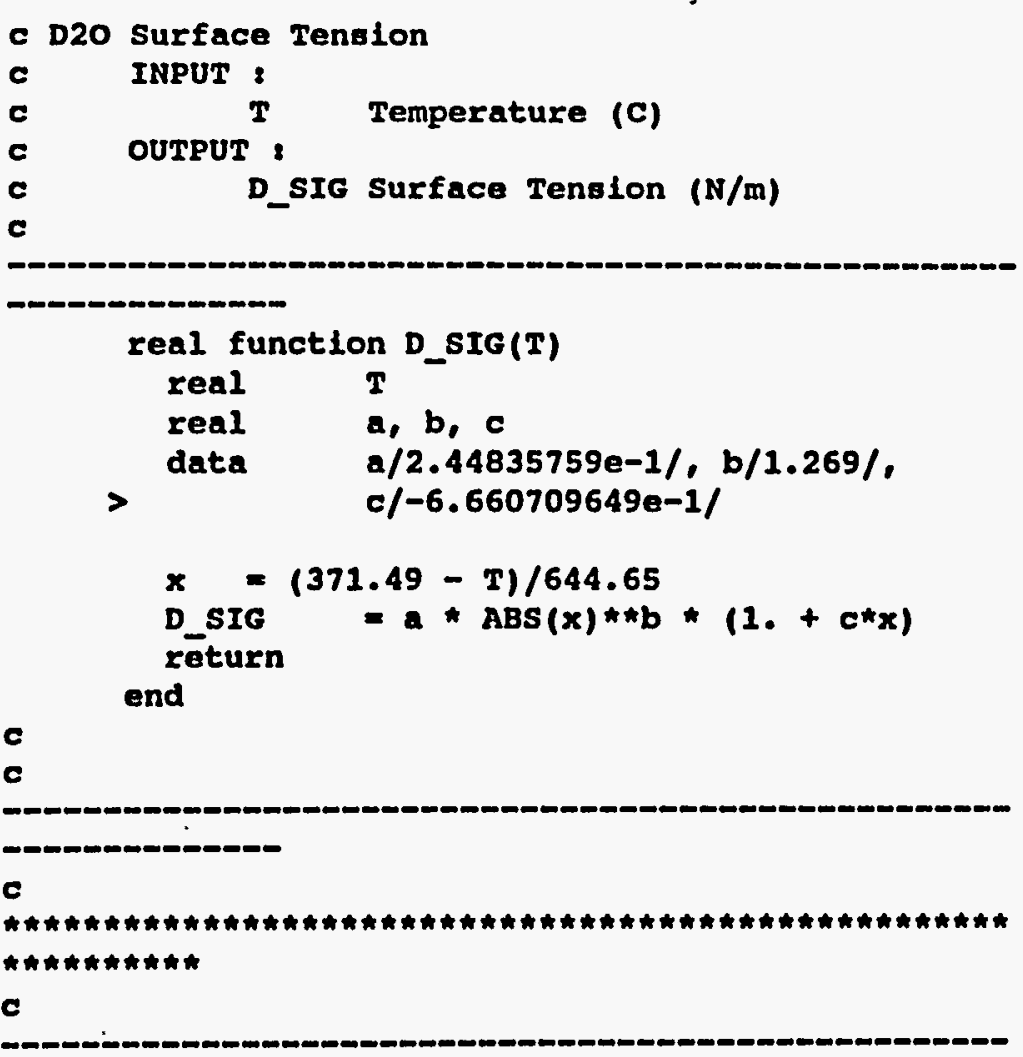

\section{c D20 Latent Heat of Vaporization}

c Note "Official" correlation uses energy in KJ. Use conv for $J$

$\begin{array}{lll}C & \text { INPUT : } \\ C & \text { Temperature (C) } \\ C & \text { OUTPUT ? } \\ \text { C }(J / \mathrm{kg}) & \end{array}$

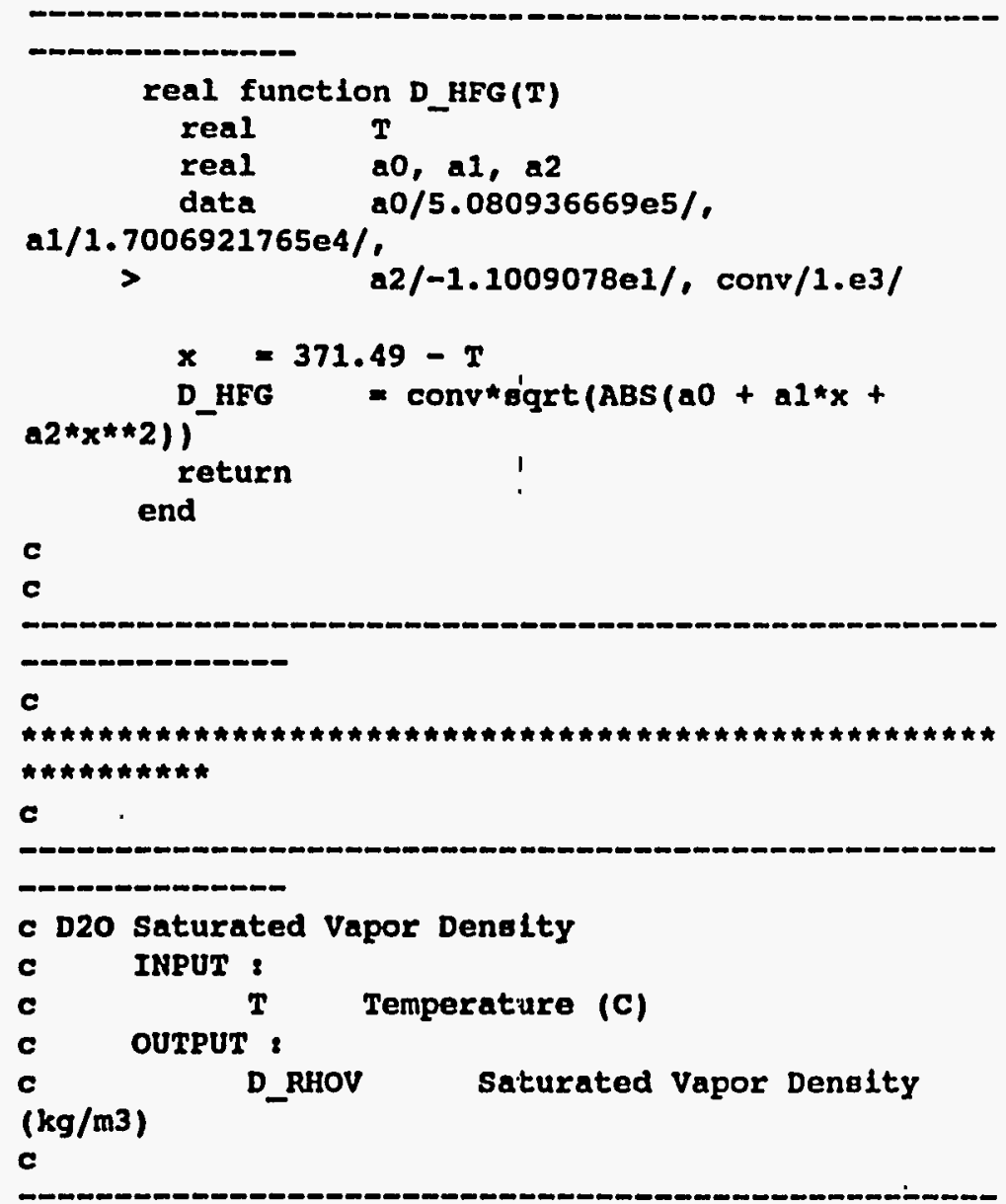

real function D_RHOV(T)

real $P$

real a0, a1, a2, b0, b1, b2

data $\quad a /-5.456208705 /$. 


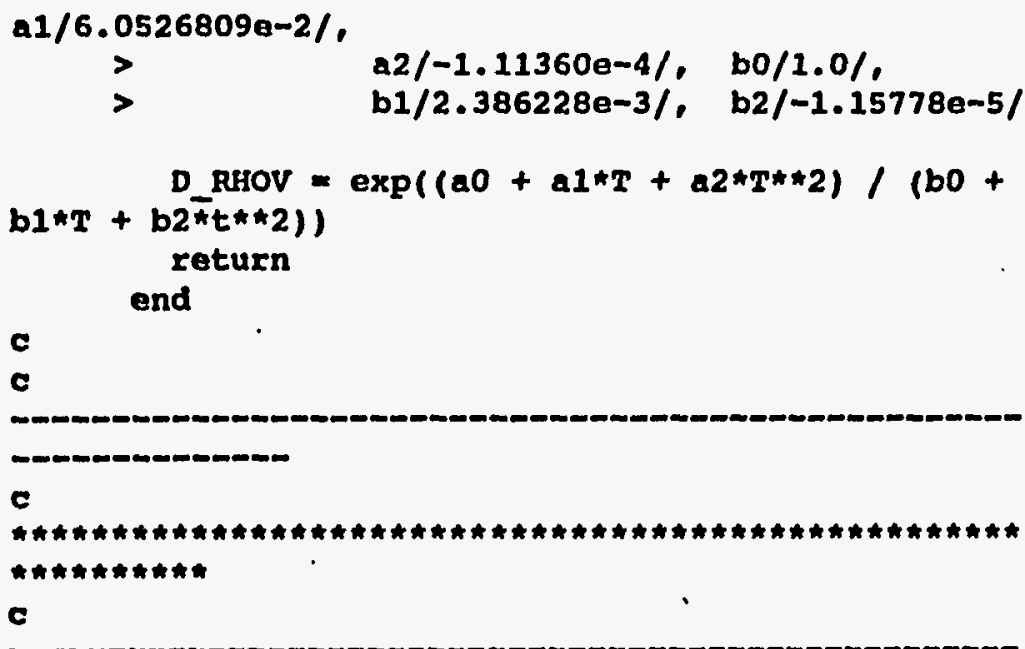

D20 Ilquid Dynamic Viscosity

c INPUT.

INPUT 8

OUTPUT :

Temperature (c)

D_MU Iiquid Dynamic Viscosity (Pa )

c

-

real function $D$ MU (T)

real in

real ao, a1, b1, b2

data $a 0 /-1.111606 e-4 /, a 1 / 9.46 e-8 /$

$>$ b1/8.873655375e-2/. b2/4.111103409e-1/

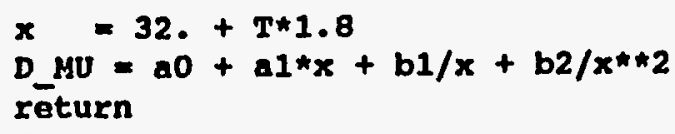

c

c

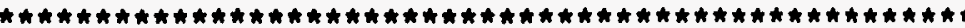

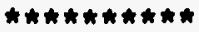

c D20 Derlvative of Ilquid Density wrt Enthalpy

c Note: "Offlclal" correlation uses energy in KJ.

Uge conv for $J$

INPUT :

$T$ Temperature (C)

OUTPUT \&

D_RH Deriv LIquid Density/ Enthalpy $(\mathrm{kg} / \mathrm{m} 3) /(\mathrm{J} / \mathrm{kg})$

c

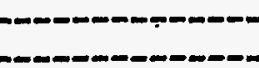

real function D RH(T)

real T

real a0, a1, a2, a3

data $a 0 /-5.5574282 e-2 /$.

al/-8.9497e-4/.

c/6.521616293e1/,

$>$

$b /-5.16987 e-3 /$

conv/1.e-3/

$c / T \star * 2)$

$D_{-} R H=\operatorname{conv}^{\star}\left(a 0+a a^{\star} T+b^{\star} \operatorname{gqrt}(T)+\right.$

return

end

c 


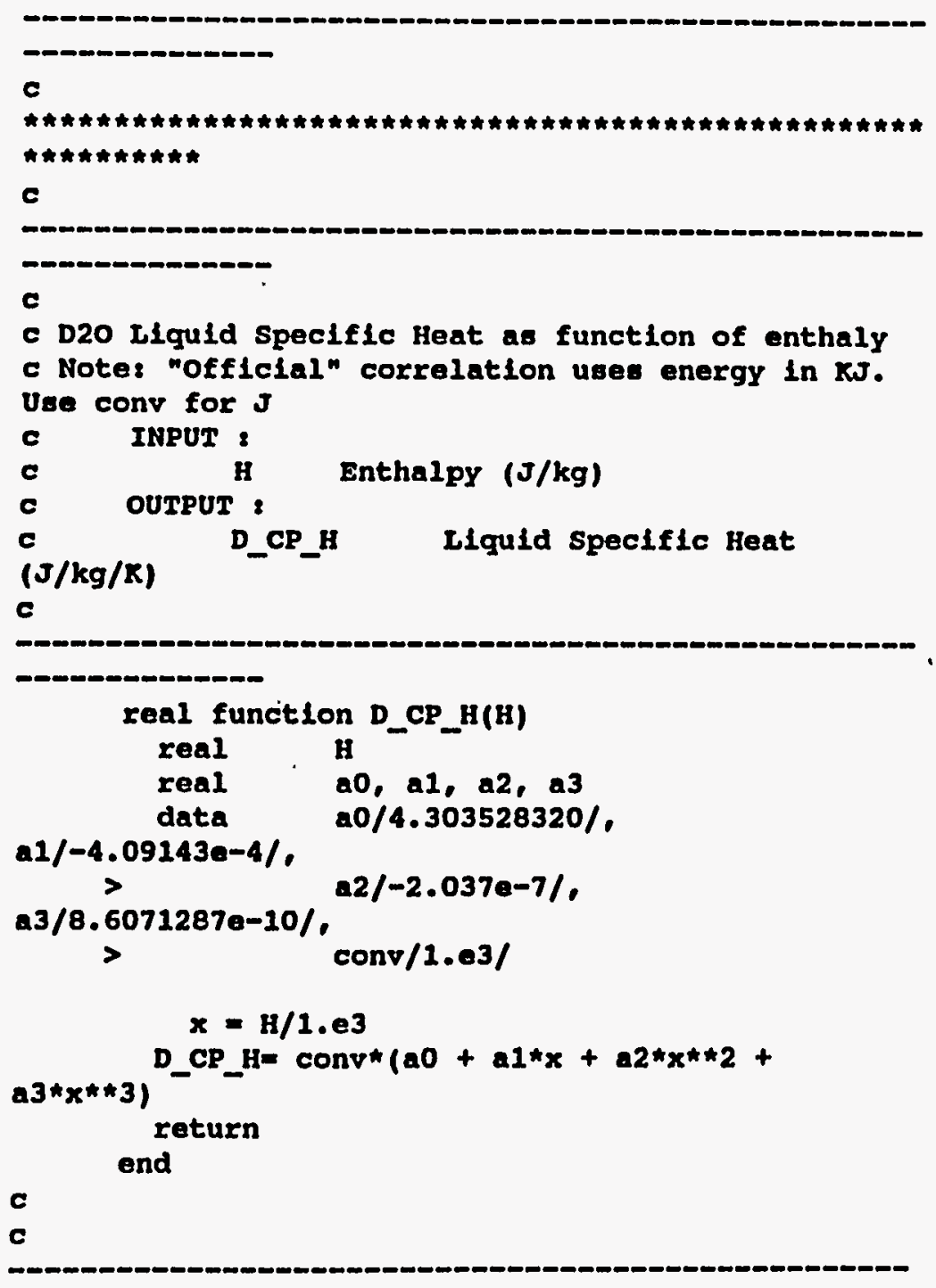

c

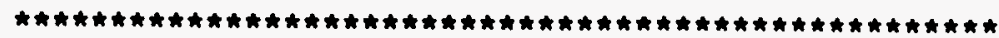

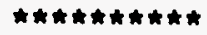

C

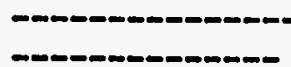

c

C D20 Saturation Preseure

C INPUT:

c T T Temperature (C)

C OUTPUT, D_PSAT Saturation Pressure (Pa)

c 

Appendix B

COOLANT-RELATED CORRELATIONS 



\section{APPENDIX B. COOLANT-RELATED CORRELATIONS}
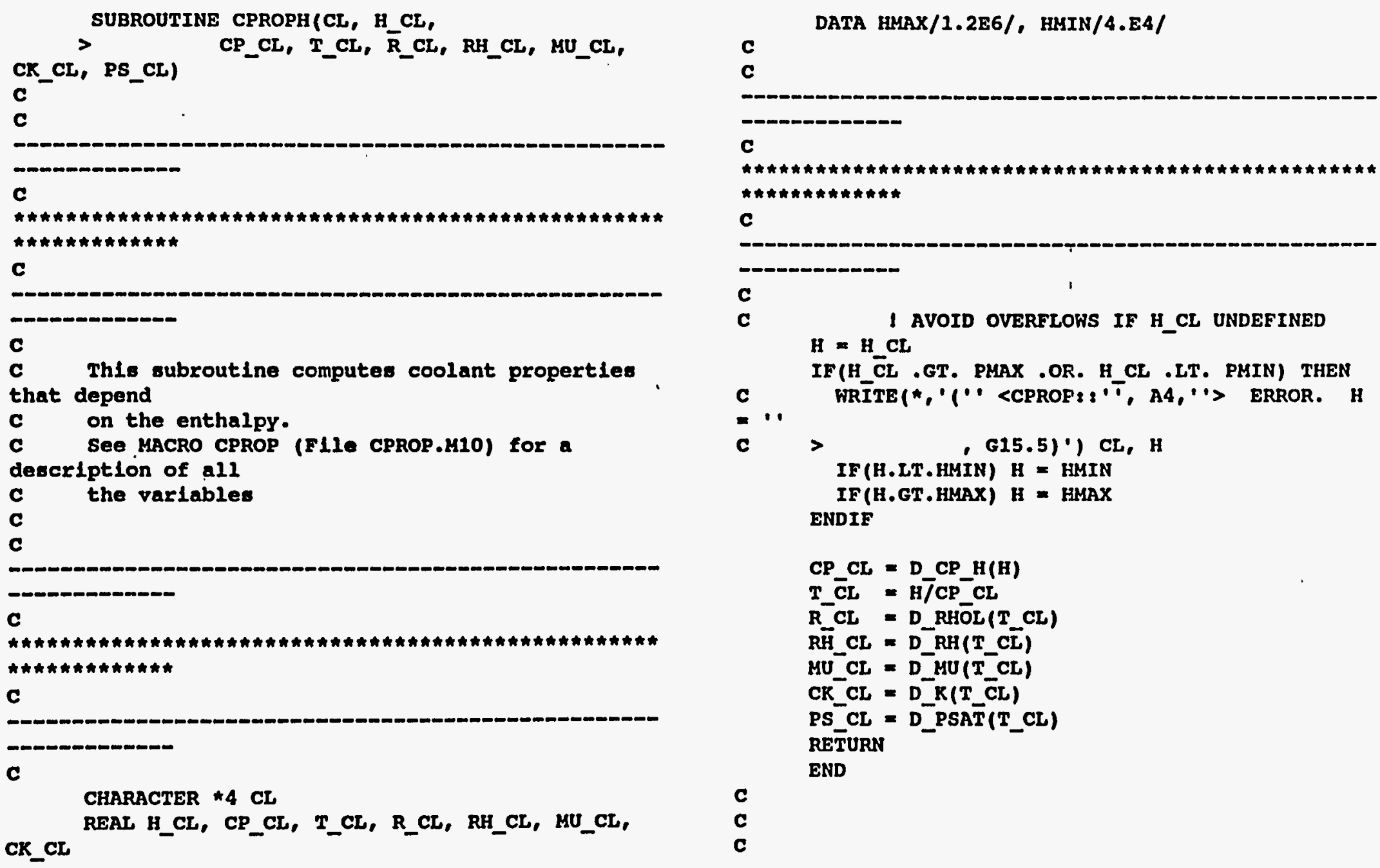
SUBROUTINE CPROPW(CL, W_CL, R_CL, KA_CL, CP_CL,

$>\quad M U \mathrm{CL}, \mathrm{CR} \mathrm{CL}, \mathrm{KD} \mathrm{CL}, \mathrm{KE} \mathrm{CL}$,

C

c

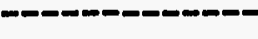

c

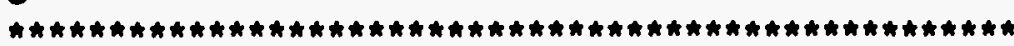

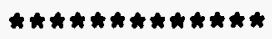

c

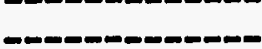

c

C This subroutine computes coolant propertles that depend

C on the flow.

c SEe MACRO CPROP (FI1e CPROP.M10) fOr a

description of all

c the variables

C

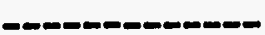

c

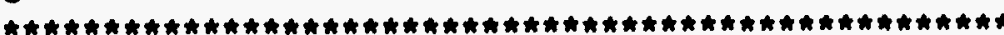
$\star * \star * \star * * * \star * \star * \star \star *$

C

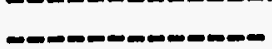

c

CHARACTER $* 4$ CI

REAL $N$ CL, R CL, KA_CL, CP_CL, MU_CL, CK_CL, KD_CL, RE_CL。

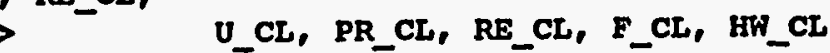
DATA RE_TUR/4240.I c

C

- - - - - -

c

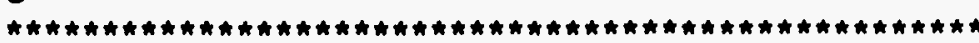

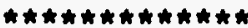

c

c

$U C L=H C L / R C L / R A C L$

$\mathrm{PR} C L=\overline{A B S}\left(\mathrm{CP}_{-} \overline{C L} * M \mathrm{HU}_{-} \overline{C L} / \mathrm{CR} C L\right)$

1. $5-4)$

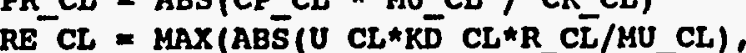

C $\quad$ core friction correlation IF (KE_CL .LE. O.) THEN

C I If smooth use Fllonenko (for

core)

IF (RE CL .GT. RE TUR) THEN

KD_CL)

$F_{-} C \bar{C} \quad F_{-} F I \bar{L} O\left(C L, R E \_C L, K E \_C L, K D \_C L\right)$ ELs $\overline{\mathbf{E}}$

E_MIN = F_FILOICL, RE_TUR, RE_CL,

F_L $=E_{-} L A M\left(C L, R E \_C L, K_{-} C L, K D \_C L\right)$

F_CL $=\operatorname{MAX}\left(F_{-}\right.$MIN, F_L $_{-}$

ENDIF

1 IF KE CL<O USE AS FUDGING FACTOR ELSE

$I F\left(K E \_C L . L T \cdot O_{0}\right) F_{-} C L=F_{-}{ }^{\star} A A B S\left(K E \_C L\right)$

C

I Else use colebrook and White IF (RE_CL .GT. RE TUR) THEN

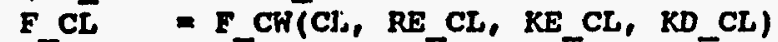

ELSE

$F_{\text {MIN }}=F_{-} C W\left(C l,, R E \_T U R, K E \_C L, K D \_C L\right)$

EL $=$ F_LAM(CL, RE_CL, KE_CL, KD_CL)

F_CL $\quad \operatorname{MAX}\left(F_{-} M I I N, F_{-} L\right)$ 


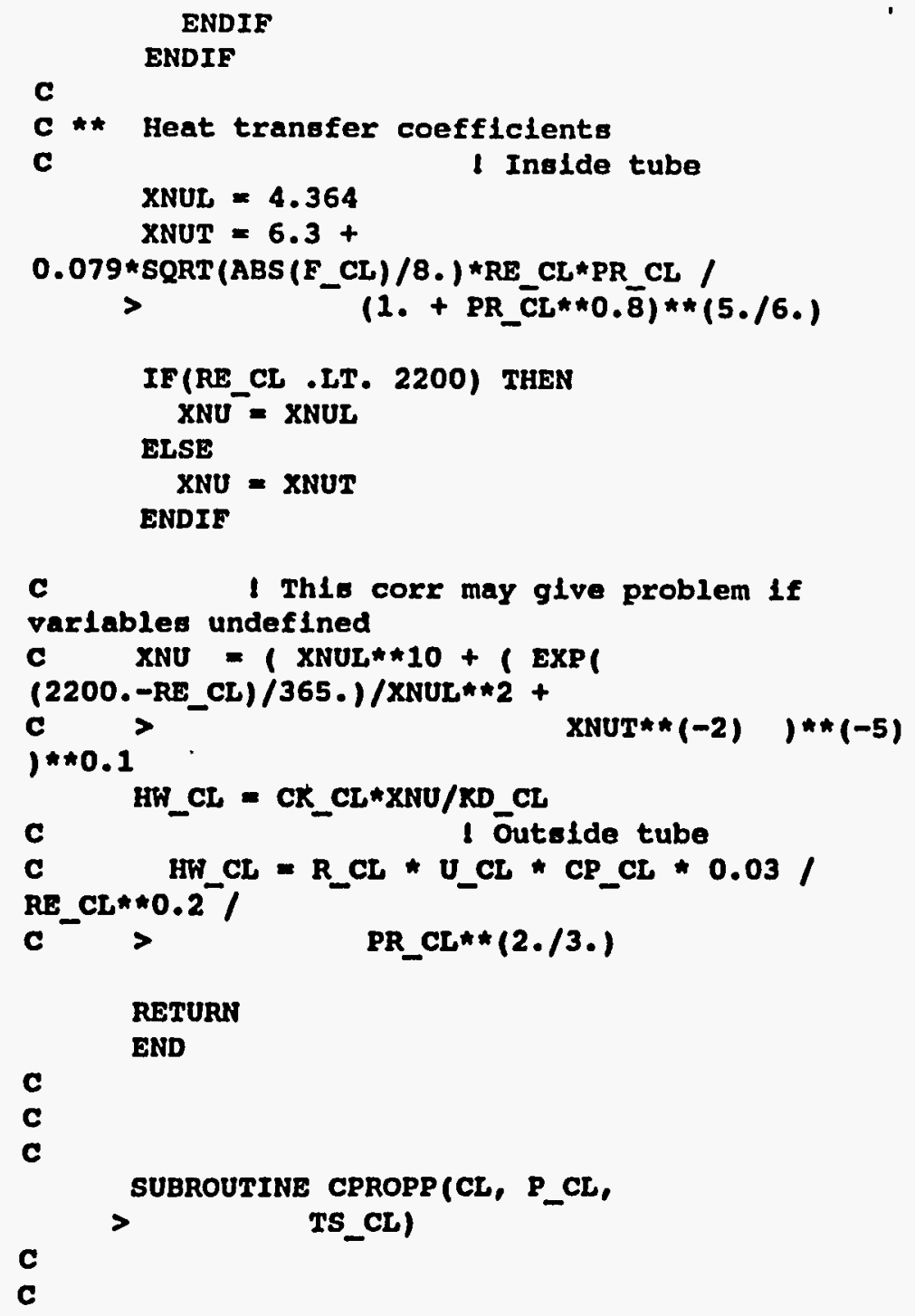

c

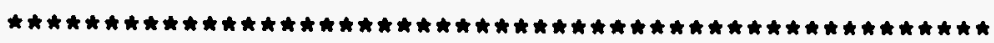

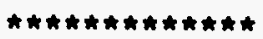

C

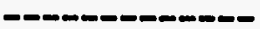

c

C This subroutine computes coolant tpropertles that depend

c on the pressure.

C SE MACRO CPROP (FIIe CPROP.M10) fOr a description of all

C the varlable:

c

C

$-$

C

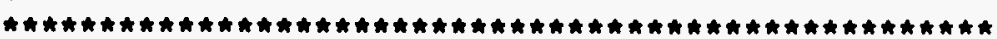

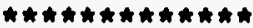

c

c

CHARACTER $* 4 \mathrm{CL}$

REAC P_CL, TS_CL

DATA PM̄IN/0.03E6/, PMAX/8.4E6/

C

c

-

c

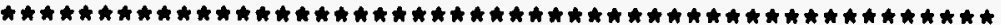

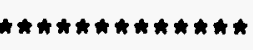

C 


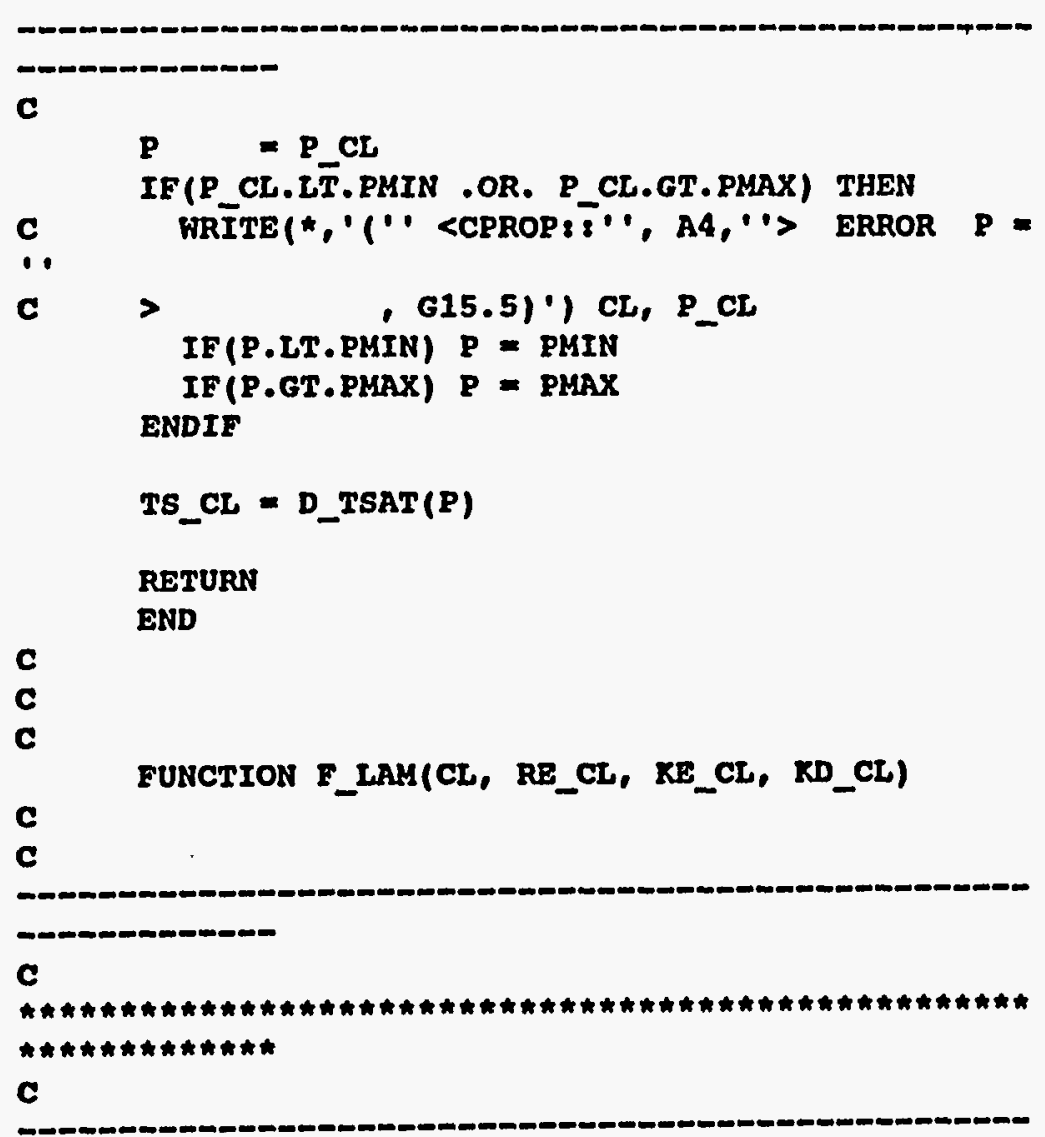

(1)

C This function estimates the laminar friction coefficient

C This function returns 4 *f

C CL C*4 Coolant node

C RE_CL $R * 4$ Reynolds number

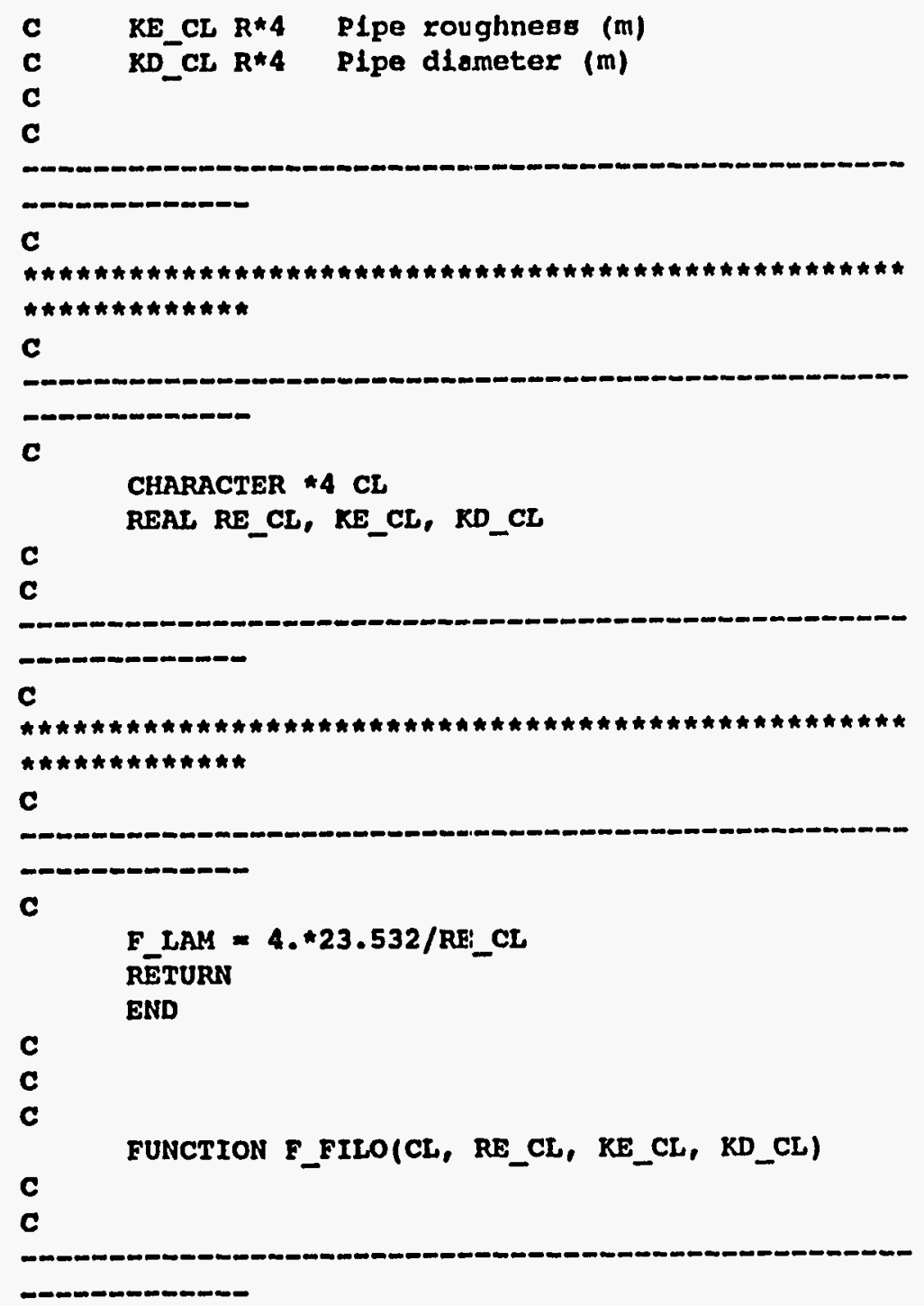


C

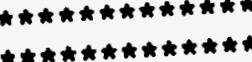

c

-

artinate the turbulent friction cosfficient

Filonenko correlation for ANS core

c based on teh rion returne $4 * f$

$\begin{array}{llll}C & \text { CL } & C * 4 & \text { coolant node } \\ C & R E \text { CL } R * 4 & \text { Reynolds number }\end{array}$

C RE_CL $R^{* 4}$ Reynolds numbex $(m)$

C KE_CL $R * 4$ Plpe diameter $(m)$

c

c

c

-

c

C

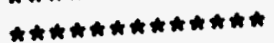

c

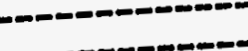

c

CHARACTER * 4 CL

REAL RE_CL, KE_CL, KD_CL

c

c

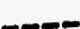

c

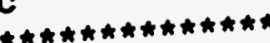

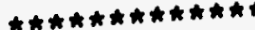

c $1.641 * * 2$

FILO $=4 . * 0.2709 /\left(1.82 * A L O G 10\left(R E C L_{1}\right)-\right.$

RETURN

C

C CH(CL, RE CL, KE_CL, RDCL)

c-nom-anam

c

C

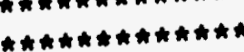

c

(10-m-

c function estimates the turbulent friction coefficlent

$c$ based on

clrcular pipes

C ${ }^{\star 4}$ coolant node

C CL $C L R^{\star 4}$ Reynolds number

C RE-CL $R^{* 4}$ Plpe roughness (m)

C KDCL $R^{\star 4}$ Pipe diameter (m)

c

c

Com- 


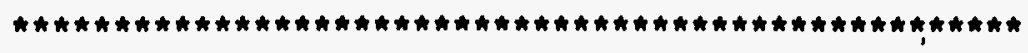

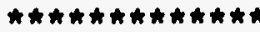

c

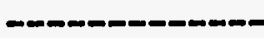

c

CHARACTER $* 4$ CL

REAL RE_CL, KE_CL, KD_CL

DATA ERT̄OR/0.0̄̄1/

C

\section{-}

$c$

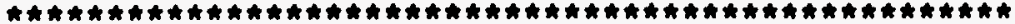

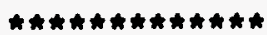

C

- -

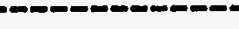

$C 1=\mathrm{RE} C L /\left(\mathrm{KD} \mathrm{CL}_{-} / 2 \cdot\right)$

$\mathrm{C2}=9 . \overline{3} 5 / \mathrm{RE}_{-} \mathrm{CI}$

C

I Moody correlation (f/4) for flrot

gues:

$\left.100 / R E_{-} C_{L}\right) * *(1 . / 3.1)$

$\overline{\mathrm{s}} \mathrm{F} \quad=\operatorname{sQRT}\left(\mathrm{F}_{-} \mathrm{M}\right)$

NITER $=20$

DO $100 I=1$, NITER

SFO $=\mathrm{SF}$

$S F=1 . /(3.48-1.7372 * A L O G(C 1+C 2 / S F))$

SFERR $=A B S((S F-S F O) / S F O)$

IF (SFERR .IT. ERROR) NITER $=0$

100 CONTINUE

F_CW $=4 . \star S E * * 2$

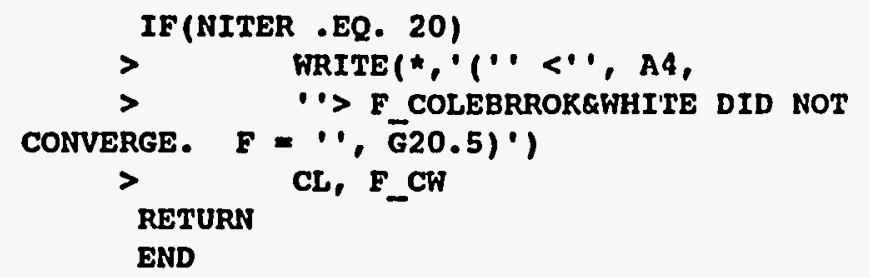


Appendix C

HEAT TRANSFER AND CRITICAL HEAT FLUX CORRELATIONS 
s 


\section{APPENDIX C. HEAT TRANSFER AND CRITICAL HEAT FLUX CORRELATIONS}
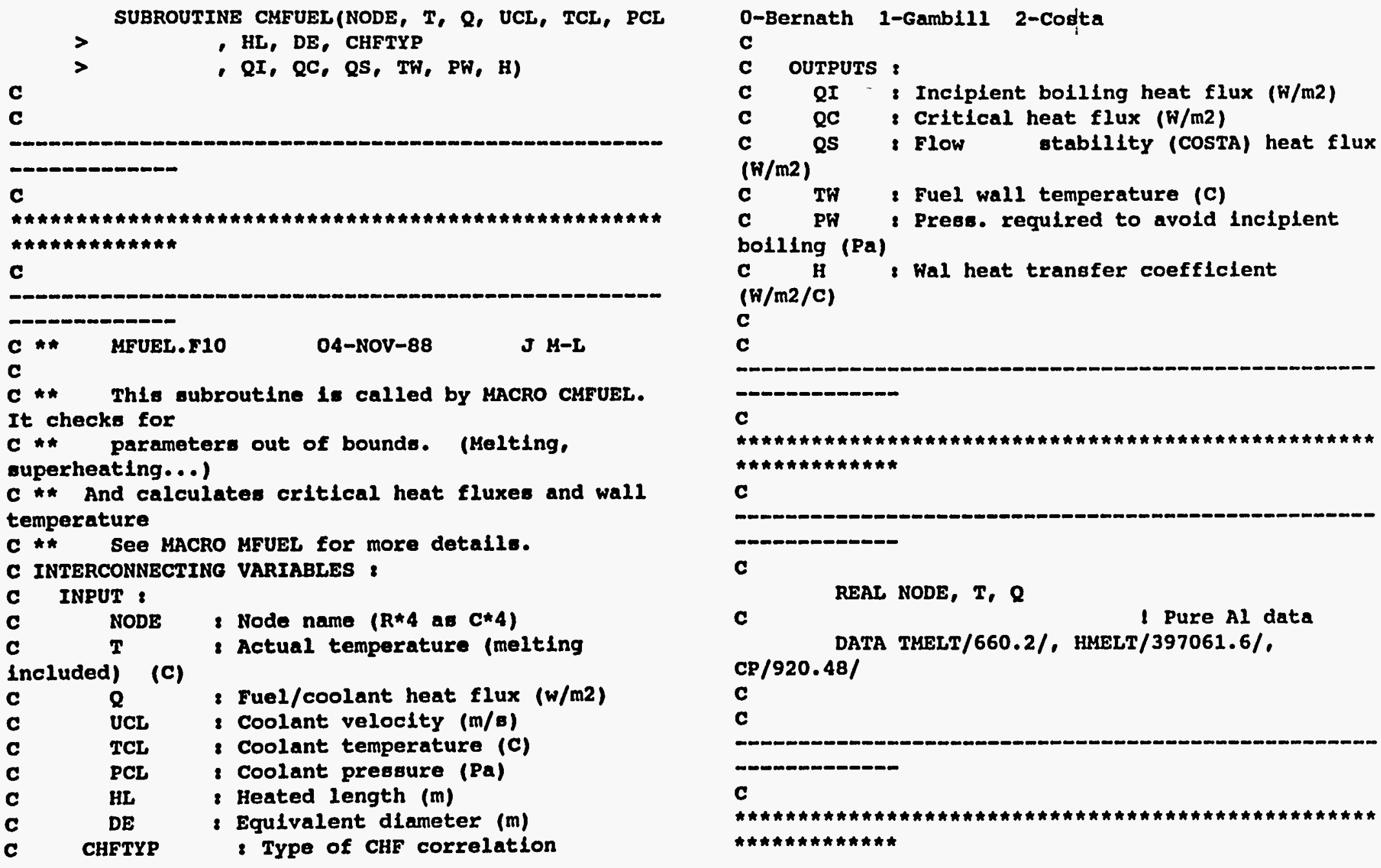$$
\text { c }
$$$$
\text { c }
$$

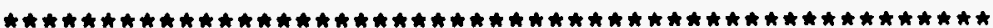

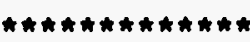

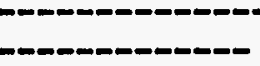

REAL NODE, $T, \boldsymbol{Q}$

c

\section{$\mathrm{CP} / 920.48 /$}

DATA TMELT/660.2/, HMELT/397061.6/.

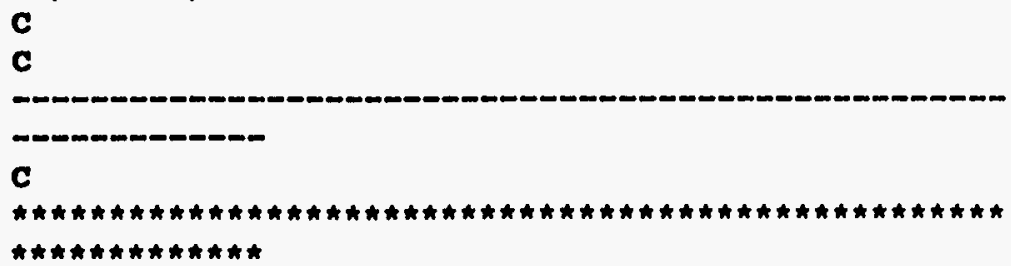


c

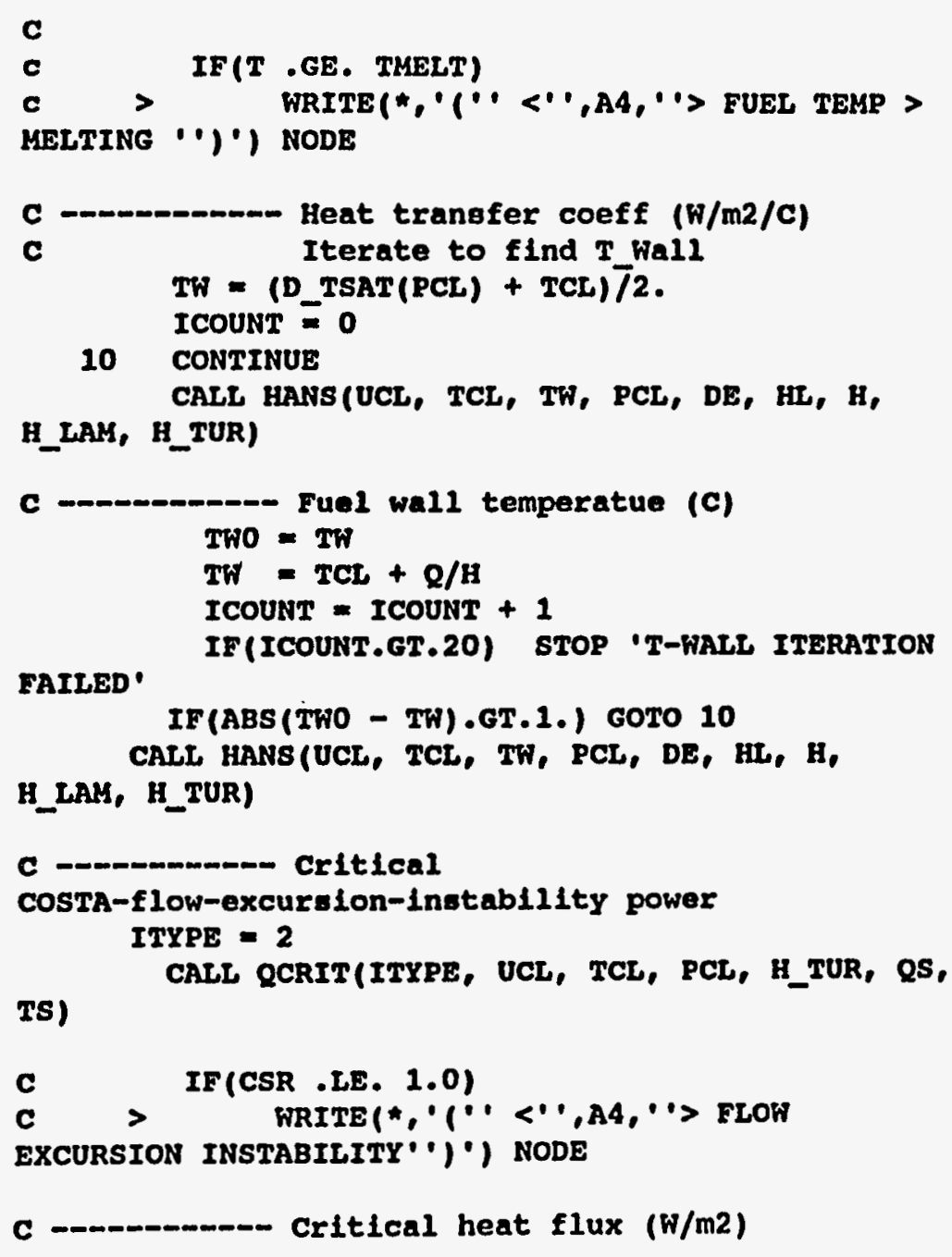

$\mathbf{c}$

c MELTING

IF (T . GE. TMELT)

C - - Heat transfer coeff $(\mathrm{w} / \mathrm{m} 2 / \mathrm{C})$

C Iterate to find $T$ Wall

$T W=$ (D_TSAT(PCL) + TCL) $/ 2$.

ICOUNT $=0$

10 CONTINUE

CALL haNS(UCL, TCL, TH, PCL, DE, HL, H, H_LAM, H_TUR)

\section{c}

- Fuel wall temperatue (C)

TWO $=T W$

$T W=$ TCL $+Q / H$

ICOUNT $=I$ COUNT +1

FAILED

IF(ICOUNT.GT.20) STOP 'T-WALL ITERATION

IF (ABS (TWO - TW) .GT.1.) GOTO 10

CALL haNS (UCL, TCL, TW, PCL, DE, HL, H, H_LAM, H_TUR)

C - -

CosTA-flow-excursion-instablilty power ITYPE $=2$

TS)

CALL QCRIT(ITYPE, UCL, TCL, PCL, H_TUR, QS,

C IF(CSR . IE. 1.0)

$C$ WRITE(*, $>(\cdots<\cdots, A 4, \cdots>$ FLOW

EXCURSION INSTABIIITY,$\%$ NODE

C - Critical heat flux (W/m2)

TC)

ITYPE - INT (CHFTYP)

CALL QCRIT(ITYPE, UCL, TCL, PCL, H_TUR, QC,

C IF (CFR .LE. 1.0)

$C>$ WRITE $(*, \cdot 1 \cdots<\cdots, A 4, \cdots>$ CRITICAL HEAT FLUX REACHED' ')') NODE

C - Preseure required to avold Incipient bolling

PW = D_PSAT (TW)

C - Inclplent bolling heat flux ( $\mathrm{H} / \mathrm{m} 2)$

ITYPE $=3$

TI) CALL QCRIT(ITYPE, UCL, TCL, PCL, H_TUR, QI,

C IF(CIR .LE. 1.0)

C > WRITE(*, ${ }^{\prime},{ }^{\prime}<, A 4, \cdots>$ INCIPIENT

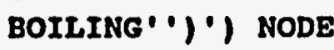

$$
\begin{aligned}
& \text { RETURN } \\
& \text { END }
\end{aligned}
$$

c

c

SUBROUTINE HANS(U, TB, TH, P, DE, HL, H, H_LAM, H_TUR)

c

C

C

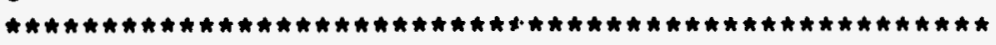

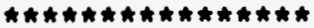

C 


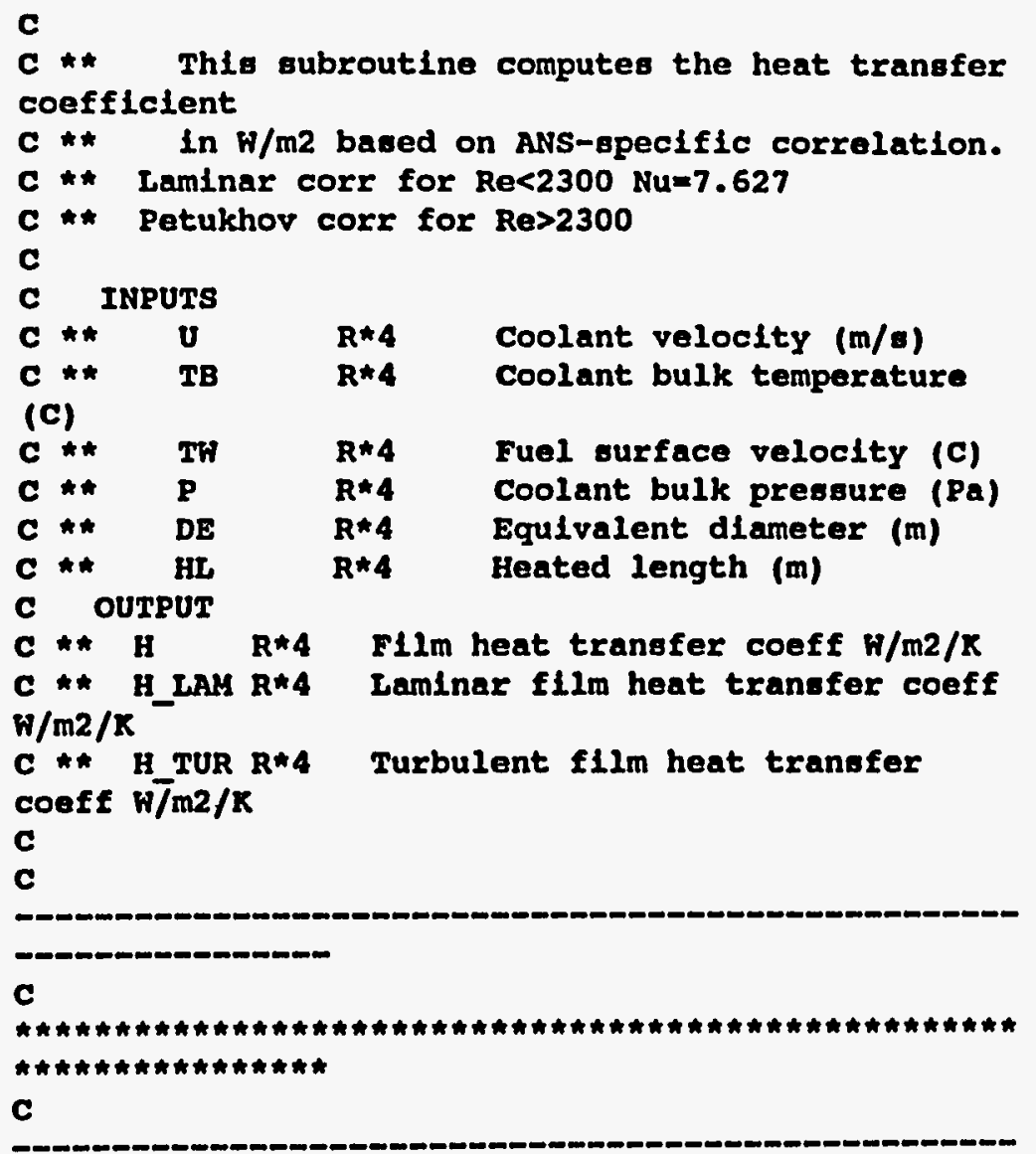

-

c

REAL U, TB, TW, P, DE, HL, H, H_LAM, H_TUR

C * Channel gap (B) and Span (S) (Avg of lowerGupper)
C

REAL B, S

DATA B/0.00127/, s/0.07882/

c

C

C

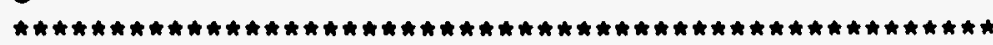

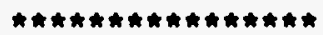

c

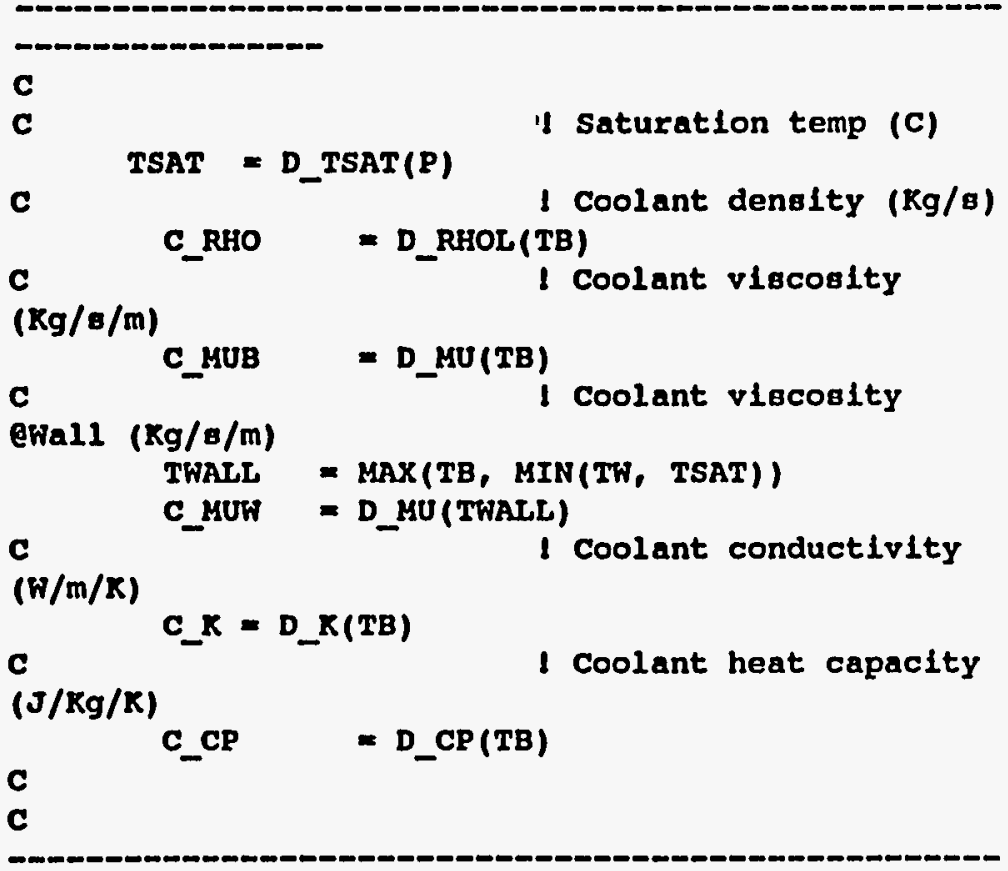

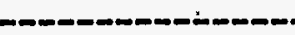

C

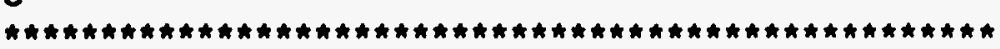




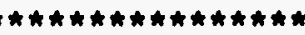

C

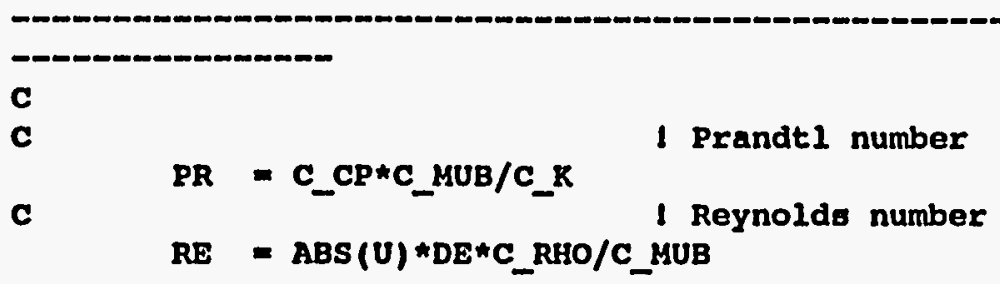

$C \star \star$ Laminar Flow (Nu $=7.627$ )

H LAM $=(C K / D E) \star 7.627$ *

(C_MUB/C_MUW) **0.11

$c *$ Transition + Turbulent Flow (Petukhov)

C* Friction factor Fllonenko

C * Fllonenko not corrected for temp for use with Petukhor

$$
\text { I } \quad(1.0875-
$$

$0.1125 * B / S) /(1.82 * A L O G 10($ Re $)-1.64) * * 2$

C $>$ (7. $-C_{\text {CUB }}$ (C_MUW $) / 6$.

c $\quad$ TUR $=($ (CKK/DE) * (F/8.) * RE* PR *

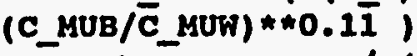

$c^{-}>$

$1.8 / \mathrm{PR} * 0.333)$

C $>\quad * \operatorname{SQRT}(F / 8) *.(P R * 0.666-$

1.) )

H NUH $=(\mathrm{C}$ R/DE) * (E/8.) * RE * PR * (C_MUB/C $\overline{M U W}$ ) $* 0.11$

$\overline{\mathrm{H}} \mathrm{D1}=(1 .+3.4 * \mathrm{~F})$

$\mathrm{HD} 2=(11.7+1.8 / \mathrm{PR} * 0.333)$

$H D 2=H D 2 \star S Q R T(F / 8$.

$H D 2=H D 2 *\left(P R^{*} * 0.666-1.\right)$

HTUR $=H_{-}^{-} \mathrm{NUM} /\left(\mathrm{H}_{-} \mathrm{D} 1+\mathrm{H}\right.$ D2)

C

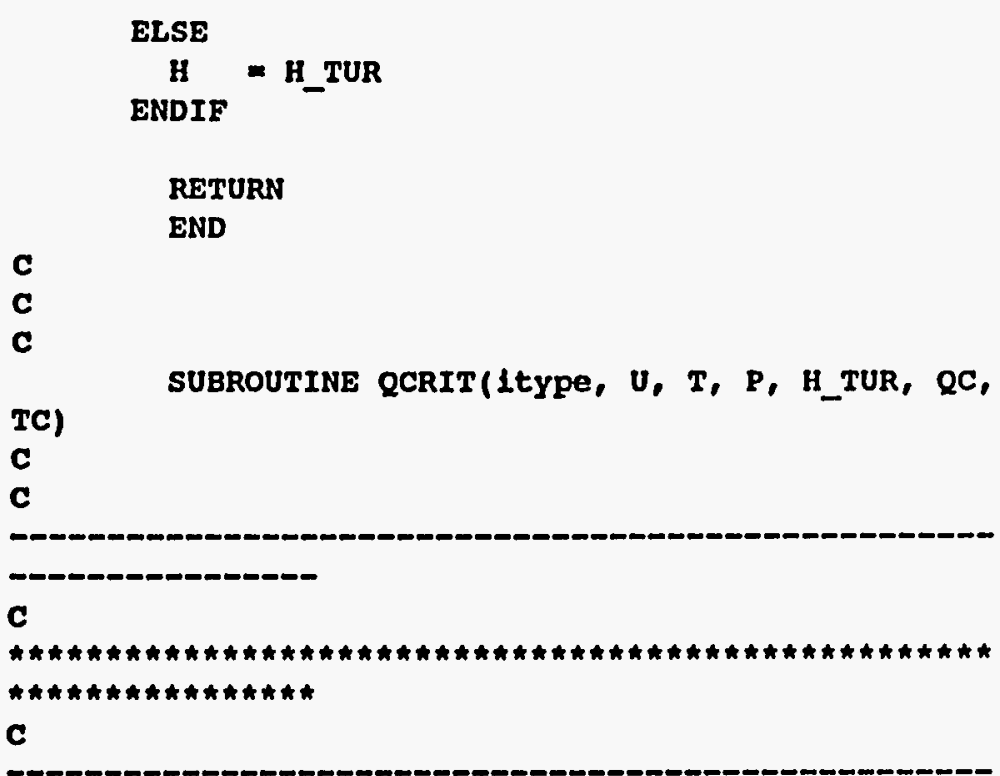

c** This function returns the critical heat flux $\ln \mathrm{w} / \mathrm{m} 2$

c INPUTS

C** Itype I*4 if 0 - Bernath

$C * *$ If 1 - Gambil1/Weatherhead

$C *$ If 2 - Cogta

$C * * U$ U $R * 4$ Coolant velocity $(\mathrm{m} / \mathrm{B})$

$C * * \quad T \quad R^{* 4}$ Coolant bulk temperature

(C) $P$ P*4 Coolant bulk pressure (Pa)

$C * * H$ TUR $R * 4$ Turbulent heat transfer coeff

$(\mathrm{w} / \mathrm{m} 2 / \mathrm{k})$

C OUTPUT

$C * *$ QC $R * 4$ Critical heat flux $\mathrm{W} / \mathrm{m} 2$ 

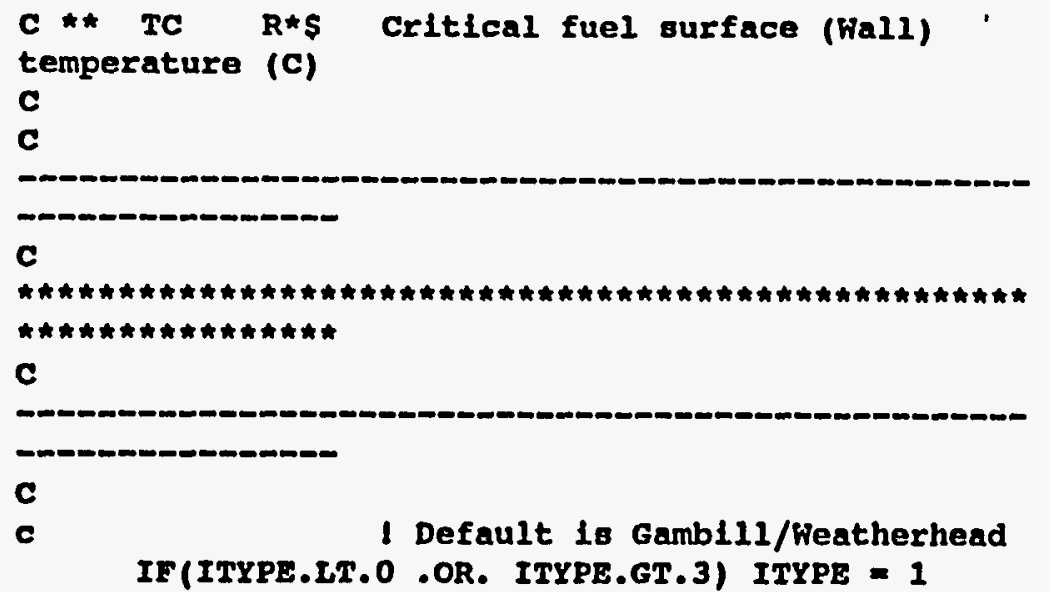

C * Bernath (Critical Heat Flux)

IF(ITYPE .EQ. O) THEN

$P M=P / 1 . E 6$

TC $=571.76-273.16+60 . \star A L O G(P M)$

$\mathrm{U} * 0.8202$ $-80.8 *(P M /(P M+0.0931))$ -

QC $=H_{-} \operatorname{TUR}^{*}(T C-T)$ RETURN

ENDIF

C * Gamb111/Weathexhead (Critical Heat Flux)

IF (ITYPE .EQ. 1) THEN $Q C=Q C G K(P, T, H$ TUR)

$T C=T \mp Q C / H$ TUR

RETURN

ENDIF

C * Costa (Flow excursion instability)

C * Noter "Offlcial" correlations are in

$\mathrm{KJ} / \mathrm{s} / \mathrm{m} 2$. Here I use $\mathrm{W} / \mathrm{m} 2$

$C * *$ Thus, the factor of 1.53 when fluxes are multiplied or divided

IF(ITYPB .EQ. 2) THEN

TSAT D_TSAT(P)

$Q C=\operatorname{SQRT}(U)^{\star}(T S A T-T) / 12.8 E-6$

$T C=T+Q C / H T U R$

RETURN

ENDIF

$C * *$ Bergles and Rosenhow (Inciplent boiling) IF(ITYPE .EQ. 3) THEN

$Q C=Q C B R\left(P, T, H_{T} T U R\right)$

$T C=T \overline{+} Q C / H_{\text {TUR }}$

RETURN

ENDIF

$\mathrm{QC}=1 . \mathrm{E}-32$

HRITE(*, $($ " selected correlation out of range $" /$

$>$

- SET CHETYP

-

1 - Gambi11

Weatherhead.

$>$

.. 2 - CoBta'

Rosenhow' ')'

3 - Bergles \&

RETURN

END

c

c

REAL FUNCTION QC_GW(P, T, H

C

$\star \star \star \star \star \star * \star * * \star$ 


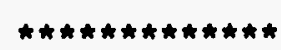

C
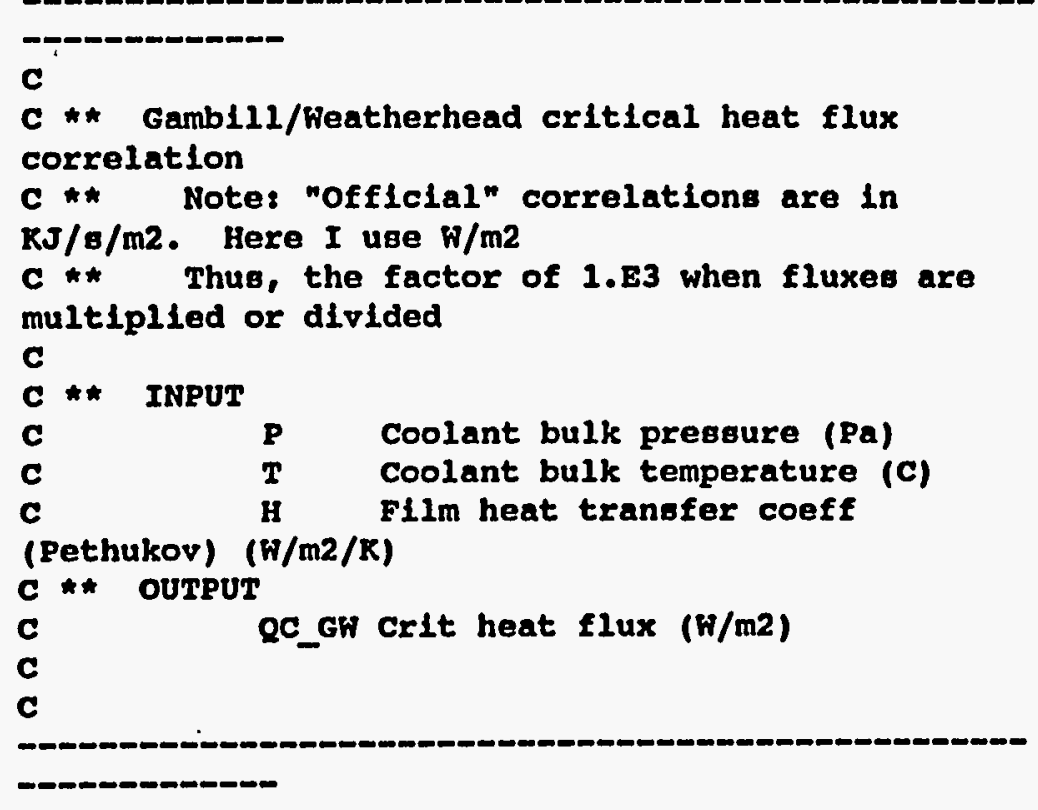

c

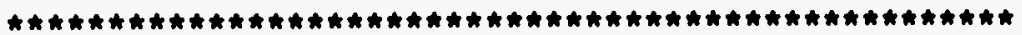

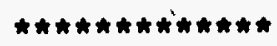

c

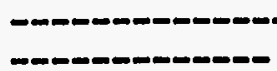

c

DATA G/9.81/, CONV/1.E-3/,

$>$ ITERMX/20/

c

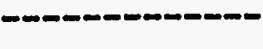

c

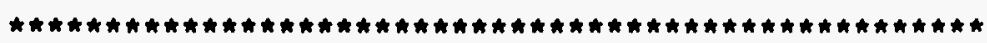

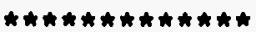

C

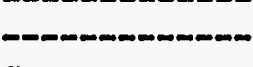

c

C.** D20 Propert1es

C HFG Latent heat of vaporization

$(\mathrm{J} / \mathrm{kg})$

C RHOL Llquid clensity at bulk coolant presenure $(\mathrm{kg} / \mathrm{m} 3)$

C RHOG Vapor density at bulk coolant

pressure $(\mathrm{kg} / \mathrm{m} 3)$

C CP specific heat $(\mathrm{J} / \mathrm{kg} / \mathrm{K})$

C SIG surface tension $(\mathrm{N} / \mathrm{m})$

C TSAT coolant saturation temp ebulk

presgure (C)

c

$$
\begin{aligned}
& \text { TSAT }=\text { D_TSAT(P) } \\
& \text { HFG }=\text { D_HFG (TSAT) } \\
& \text { RHOL }=\text { D_RHOL (TSAT) } \\
& \text { RHOV }=\text { D_RHOV (TSAT) } \\
& \text { CP }=\text { D_CP(TSAT) } \\
& \text { SIG }=\text { D_SIG (TSAT) }
\end{aligned}
$$

c

$c$ " Correlation 1s only valid for subcooled flow

$c$

$$
\text { IF (T . GE. TSAT) THEN }
$$

$$
\text { QC_GW }=-1 . \mathrm{E} 32
$$$$
\text { RETTURN }
$$

$$
\text { ENDIE }
$$

1 pool crit heat flux in $\mathrm{w} / \mathrm{m} 2$

$\mathbf{c}$

2 POOL

$0.18 * H F \bar{H} \star R H O V *(S I G \star G \star(R H O L-R H O V) / R H O V \star \star 2) \star \star 0.25$

$>$

*(1. + (RHOL/RHOV $) \star * 0.75 *$ 
C I Initial guess use crit $t$ wall without

Heatherhead correction

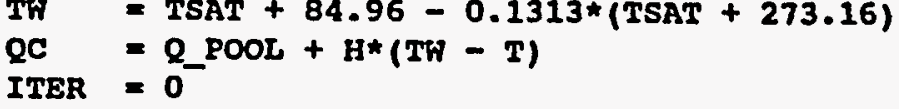

10 CONTINUE

$$
\text { ITER = ITER + } 1
$$$$
T H=147.7-
$$

$0.127 *$ TSAT $) \star A B S(2 C / 3.1546 \mathrm{E} 6) \star 0.25+\mathrm{TSAT}$

$$
\text { OLD QC }
$$$$
Q C=Q \text { POOL }+H *(T W-T)
$$

IF (ITER.GT.ITERMX) THEN WRITE(*, 1 " I 1 ERROR in cxit heat flux Lteration' ') ')

$$
\begin{aligned}
& \text { QC_GW } \\
& \text { RETURN }
\end{aligned}
$$

ENDIF

IF (ABS ( (QC - OLD_QC)/QC) . GT.CONV) GOTO 10

QC_EN $=Q C$

RETURN

END

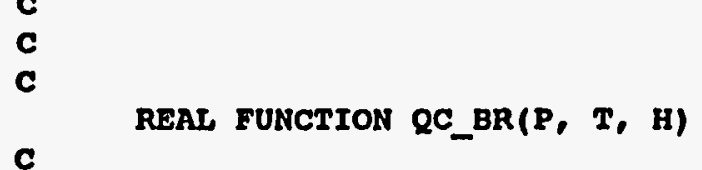

C

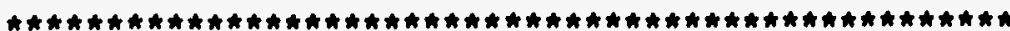

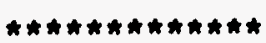

c

C * * Bergles \& Rohsenow Inclpient bolling heat flux correlation

C * Note: "Official" correlations are in

$\mathrm{RJ} / \mathrm{s} / \mathrm{m} 2$. Here I use $\mathrm{N} / \mathrm{m} 2$

$C * *$ Thus, the factor of 1.83 when fluxes are multiplied or divided

c

$C \star \star$ INPUT

C P Coolant bulk pressure (Pa)

C T Coolant bulk temperature (C)

C H Fllm heat tranefer coeff

(Pethukov) ( $\mathrm{H} / \mathrm{m} 2 / \mathrm{K})$

C *

$(\mathrm{H} / \mathrm{m} 2)$

C

(1)

C

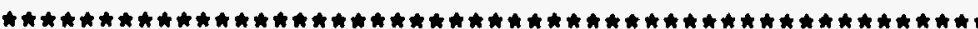
$* \star * * * * * \star * \star * \star * * *$

C

(1)

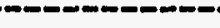

C

DATA CONV/1.E3/, ITERKX/20/

c

C

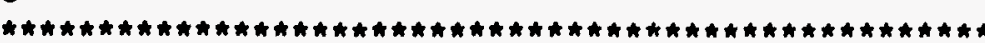




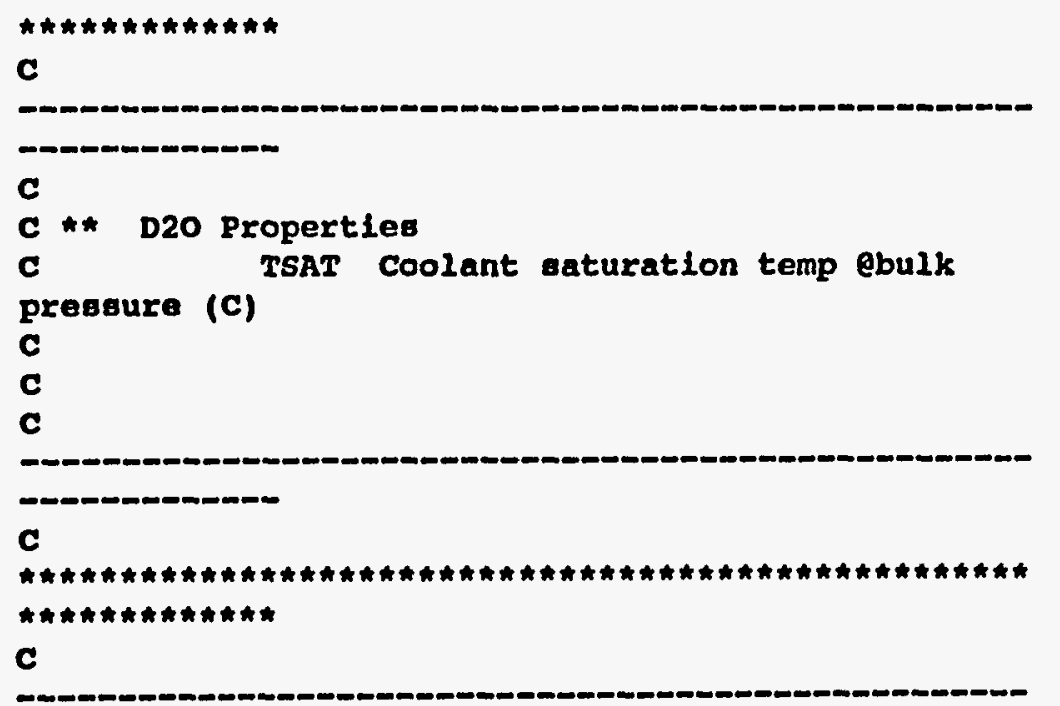

-

c

$$
\text { TSAT = D_TSAT(P) }
$$

C C1.

$3.1546 * 0.98 * 15.6 *((P / 6.8948 E 3) * * 1.156)$

C $C 2=(2.3 /(2 / 6.8948 \mathrm{E} 3) * 0.0234)$

C1 $=$ CONV $0.9 * 1.7978 \mathrm{E}-6$ *

$(P) \star * 1.156$

$\mathrm{C2}=2.8285 /(P) * 0.0234$

$\mathrm{TC}=\operatorname{TSAT}+10$.

TW $=$ TC

NITER $=0$

10 NITER $=$ NITER +1

QC $=C 1 *(1.8 * A B S(T W-T S A T)) * * C 2$

$T C=T+Q C / H$

$F=T H-T C$

DFDT $=1 .-\mathrm{C} 1 \star \mathrm{C} 2 / \mathrm{H} \star 1.8 *$

$(1.8 *$ ABS $(T W-T S A T)) * *(C 2-1$.
$D T=-F / D F D T$

IF (NITER . LT. ITERMX) THEN IF (ABS (DT) .GT . 0.01) J.HEN

$T W=T W+D T$

IF (TW .LT. TSAT) TW $=$ TSAT + 1 . GOTO 10

ENDIF

ELSE

FRITE(*, ${ }^{\prime}$ ' Inclpient bolling lteration not converged' '/')

$$
\text { QC }
$$

ENDIF

\section{$Q C B R=Q C$}

RETURN

END 


\section{Appendix D}

STANDARD MACRO DEFINITION FILE (ANS.CMD) FOR THE ADVANCED NEUTRON SOURCE DYNAMIC MODEL 



\section{APPENDIX D. STANDARD MACRO DEFINITION FILE (ANS.CMD) FOR ANSDM}

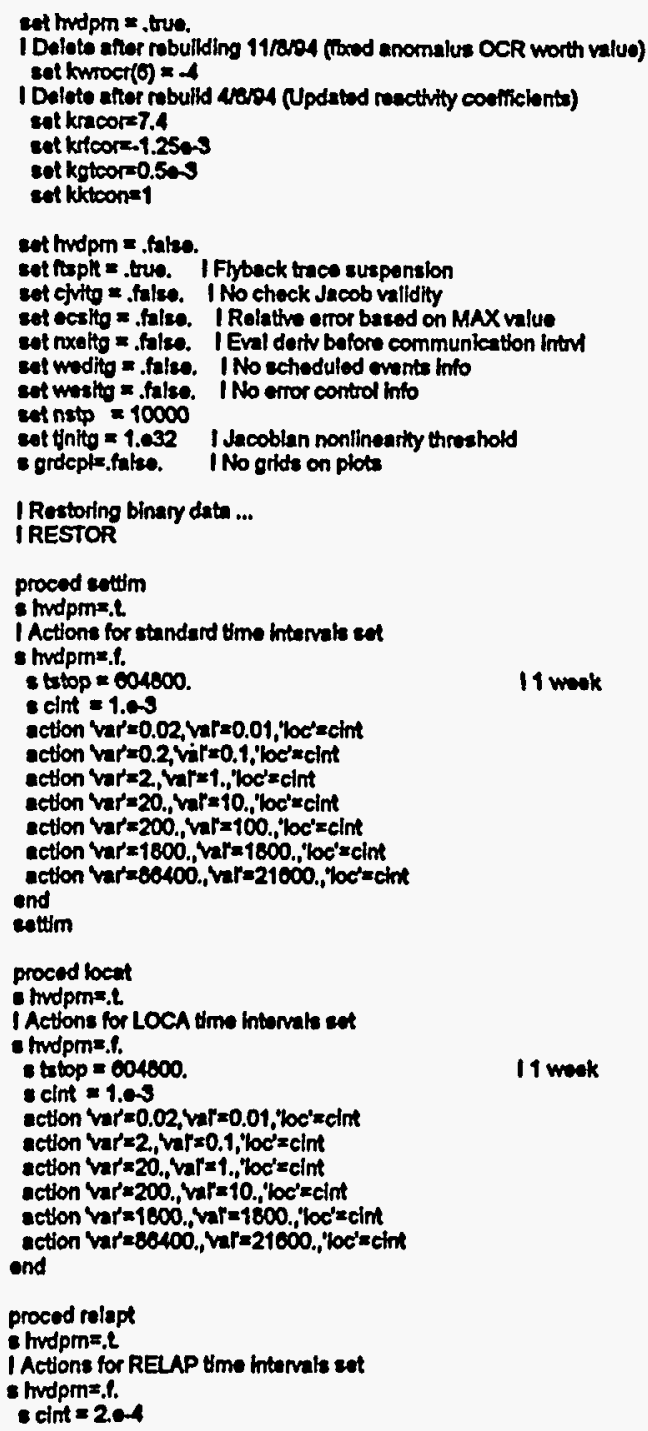

11 mook

- trtop= 25

action Var $=0.01$, var $=0.001$, $\% 00=$ int

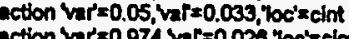

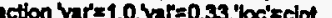

ection varses go var =0.

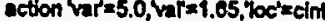

action var $=24.0$, var $=0.2$, toc $=\mathrm{dnt}$

and

prepar 'clear.t.

propar pvest,pvoco,pmepl,pmepo

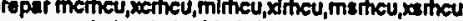

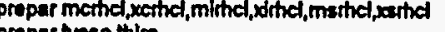

propar twero, thilo

prepar tmcpi,tmepo, teiri, imost

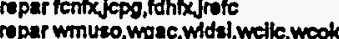

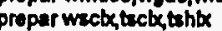

propar natem, nopol, repol

propar sice, iticer

\section{1 proced go tan}

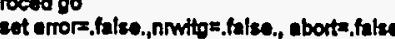

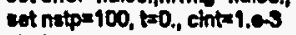

end

1 mutiplo rum

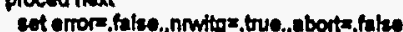

ent matp=100, t=0., cint = 1.e.

ind

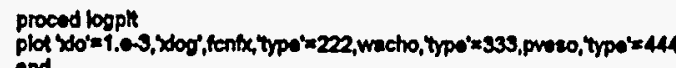

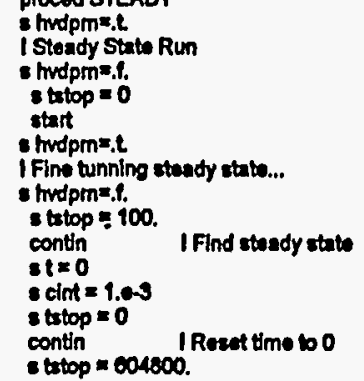

procod ppeof
stmodprn=.l

i Plunt protoction

atudponnt.

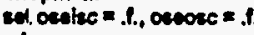

end

proced ppeon

Imodpm=t.

Plamt protection system on

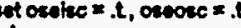

procod noreon

Rods will not movo.

proced roll

IExtemal resectivity coet to zero

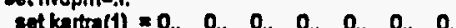

cot kertra(11) $=0$. , 1.010, 8.010, 1.010, 1.010, 1.010, 1.010

procend ron

cet hudpmin.t.

IExtermal reactivity eot to 1.0 dol stop (n $1 \mathrm{~ms}$ )

cot kartra (11) $=0 ., 0.001,1,010$

proogd p2off

iserondary pump speod will tip to nat circ. at t $=0$

I Control para KKTCON and KTNCON have been changed.

cet hivdpm=.f.

ection var $=0$, var $=0.0$,'toc' $=k$ nocon

skition $=0$

introon $=5$

proasd pony

wil be cet to pony speod $(10 x)$ al time 0

cet hudpme.f.

ection var' $=0$, var 0.1 , 'loc'sznomep

end

procod nateir
sot hudpm $=\mathrm{L}$

I Pry pump epeed will be set to 0 (natural circulation) at time 0 


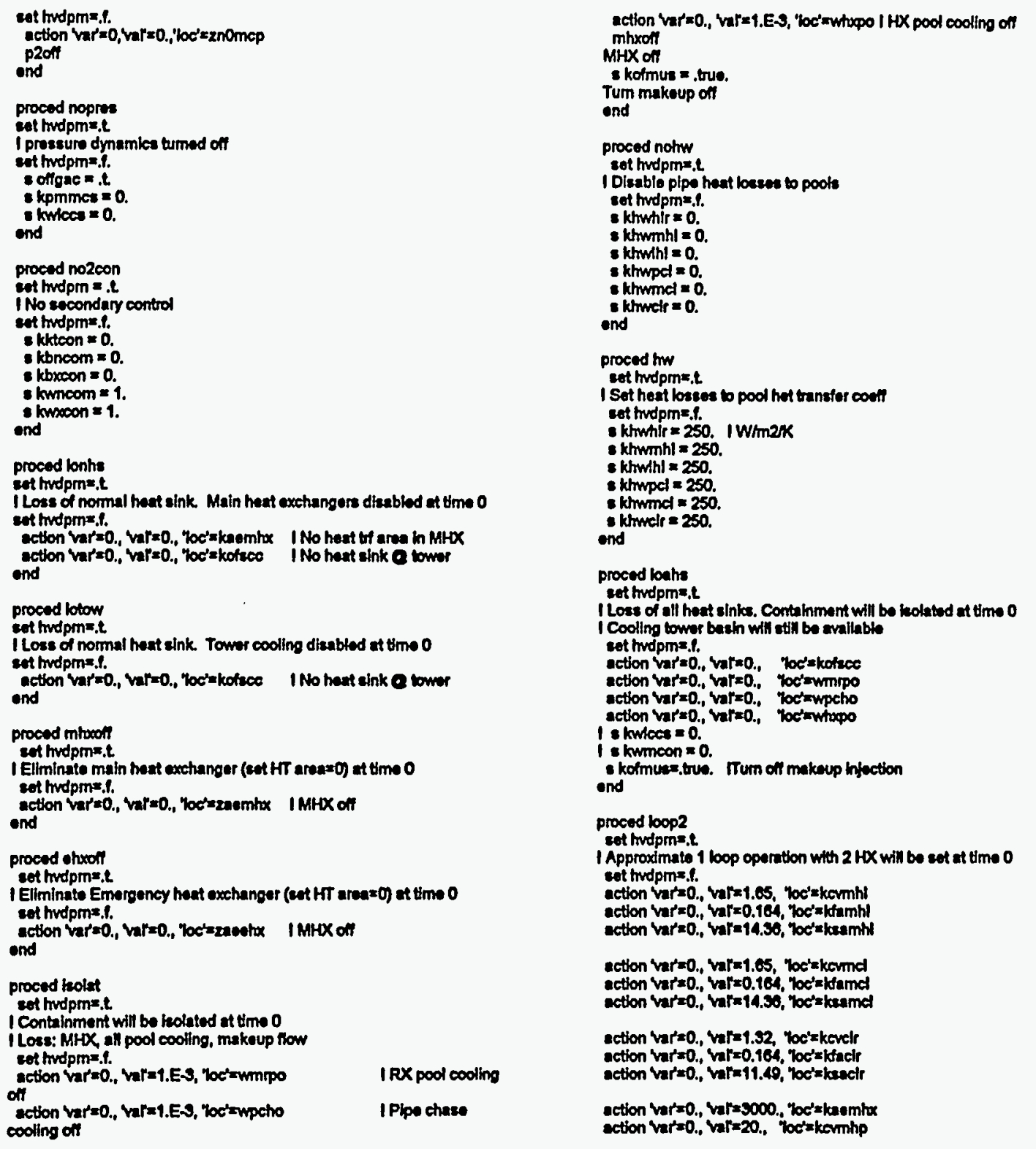

action var=0., var $=1.25$, 700'zktamhp

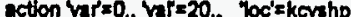
ection var'=0., var=1.15, 4oc'zklashp

ection var $=0$, var $=250$., "loc' $=$ keveehx

ection var $=0$. , var" $=12$. "loc'zkevthl

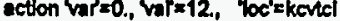
ection var $=0$. , var $=0.50$, "loc'=kfalcl

ection var $=0$. var $=3000$., Yoe'zeketh

ection var=0., var:150., 'loc'=kevpch

cet knphes $=0.05 \quad$ I Pony speed (10\%/2) anter zeram

end

proced ecram

| Reactor ecram roquent sel at $1 \mathrm{~ms}$. Dolay still activo

cot hodpme.f.

end

procod nollm

I Control rod ilmits of cervo motion removed

cot thudpman.f.

$s$ lositer $=1.010$

ind

proced nolpol

- Klipol = 0.1 No Inittal lodine

- Kupoi $=0$. I No Initial Xonon

proced dlgeon

I Digital control active. DTFCON, DTPCON, DTTCON ent eampling

I Digh

tot thudpm = . f.

cot todccon $=$. to tue

cot OTFCCN $=0.01 \quad$ I Power (mux) control sampling tme

set DTPCCN = 0.5 i Prossure control eamping tme

cot DTTCCN $=0.5$ ITemperature control eampling time detteon, apcon,

PROCED USS

cot thdprn:n.t.

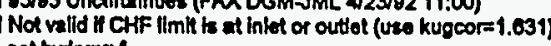
cot hodprn=.1.

- Khueor $=1.074$

kugeor $=1.554$
kucoor $=1.305$

1 Hot streak uncertainty Hot spot unc. for CHF 
PROCED UPO

109.900 Uncertaintios (FAX DGM JML A23502 11:00

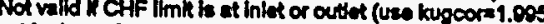
est indprn=.1.

\begin{tabular}{|c|c|}
\hline $\begin{array}{l}\text { s khucor }=1.113 \\
\text { s kugcor }=1.800 \\
\text { s kuecor }=1.502 \\
\text { END }\end{array}$ & $\begin{array}{l}\text { Hot streak uncertuinty } \\
\text { i Hot spot unc. for CHF } \\
\text { I Hot spot unc. for FE m }\end{array}$ \\
\hline
\end{tabular}

PROCED UMULT

sot modprn=.L I Multiplicative uncertalntios (OId HFIR typo) unod JMLL PO 72

\begin{tabular}{|c|c|}
\hline $\begin{array}{l}\text { khucor }=1.259 \\
\text { kugcor }=1.40 \\
\text { kucoor }=1.46 \\
\text { NDO }\end{array}$ & $\begin{array}{l}\text { I Hot etreak uncertainty } \\
\text { I Hot spot une. for CHF } \\
\text { I Hot spot unc. for FE. m? }\end{array}$ \\
\hline
\end{tabular}

PROCED UPS2

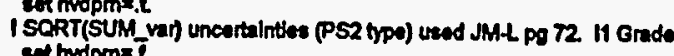

\section{8 khucor $=1.144$ \\ kugeor $=1.32$}

IHot strank uncertininty

END i Hot epot unc. for FE ?

PROCED UNO

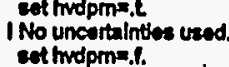

\begin{tabular}{|c|c|}
\hline $\begin{array}{l}\text { Ehucor }=1.0 \\
\text { kugcos }=1.0 \\
\text { sucos }=1.0\end{array}$ & 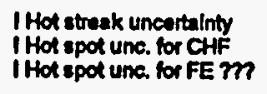 \\
\hline
\end{tabular}

PROCEDLIBOC

ent hudprnz.l

I Boginning of Cyelo detalted pown shapes (27 nodes)

IL Lower core 80C. II

kpleor $=1.390$

- knntict $=27$

$=1.215,1.368,1.407,1.301,1.366,1.382,1.306 \ldots$

$1.980, \ldots$

$1.374,1.373,1.364,1.372,1.373,1.302$.

$1.270, \ldots$

$1.301,1.307,1.401,1.402,1.350,1.269$

$1.210,1.164,1.114,1.036,0.026,0.793$

I Upper core BOC. II

kpucor $=0.041$

Kpathey $=0.701,0.834,0.890,0.010,0.890,0.87,0.855$

0.014

$0.880,0.792,0.090,0.079,0.058,0.037$

$0.597,0.570,0.503,0.547,0.525,0.500$
$0.407, \ldots$

END

$0.467,0.450,0.455,0.420,0.380,0.350$

PROCED IIEOC

IEnd of orele detalied power chapese (RT nodet)

ent indprin=.1.

ILower core EOC. II

kploor $=0.092$

B kpethet $=0.405,0.502,0.529,0.520,0.500,0.463,0.474 \ldots$

$0.306, \ldots$

$441,0.427,0.412,0.406,0.309,0.390$

$0.423, \ldots$

$0.403,0.407,0.415,0.423,0.416,0.412$

IUpper core EOC.

- kpucor =1.250

Knnheu $=27$
Kesheu
$=0.877,1.119,1.281,1.419,1.420,1,431,1.430, \ldots$

$1.555,1.416,1.261,1.280,1.286,1.263,1.270, \ldots$.
$1.261,1.252,1.241,1.220,1.190,1.165$

$1.140, \ldots$

$$
1.264,1.252,1.241,1.220,1.189,1.105 \text {, }
$$

ENO

$1.117,1.120,1.143,1.147,1.207,1.330$

PROCED I1

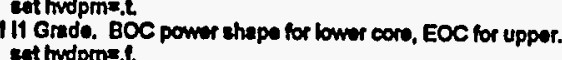

IUpper core EOC. I1

- knnthes $=27$

I kptheu $=0.877,1.119,1.281,1.410,1.420,1.434,1.430$

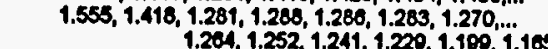

$1.140, \ldots$

$1.117,1.120,1.143,1.147,1.207,1.330$

ILower core BOC. 11

$\begin{array}{ll}\text { knnhet } & =27 \\ \text { kpshel } & =50^{\circ}\end{array}$

\& kpthet $=1.215,1.368,1.407,1.391,1.380,1.382,1.390, \ldots$

$1.270, \ldots$ $1.394,1.397,1.401,1.402,1.350,1.280$.

END $1.210,1.164,1.114,1.030,0.020,0.793$

PROCED D

$13 \mathrm{Grdpdo}$. EOC

sot hwodpmas.

1 Upper core EOC. B

kpUcor $=1.531701$ EOC. To agroe wth RELAP $\$ W W / \mathrm{m} 2$
$=5$
- kpsheu $=50^{\circ} 0$

$=1.362,1.014,1.566,1.517,1.517$

ILower corr EOC. 13

8 kploor $=0.006501$ EOC. To egreo with REA AP MW/m2 kpethel $=50^{\circ}$

Kothed $=1.100,1.034,0.003,0.001,0.804$

PROCED M1

IMI core power distributions.

8 kpucor $\approx 1.417174$

Knnhicu $=35$

8 1133:303E+01, . 144805E+01, .150300E+01, .171713E+01, .152007E+01, 142840E+01, 140383E+01, 145001E+01, $141338 E+01$

$.155028 \mathrm{E}+01, .150702 E+01, .155000 \mathrm{E}+01, .150002 \mathrm{E}+01$,

$150300 \mathrm{E}+01$,

$.101309 E+01, .158341 E+01, .102455 E+01, .158724 E+01$, $.167873 E+01, .144018 E+01, .140452 E+01, .152382 E+01$, $.121482 E+01, .122511 E+01, .124843 E+01, .122010 E+01$ $.128158 E+01$, $.105417 E+01, .028000 E+\infty 0, .102023 E+01, .053287 E+\infty 0$ DS6873E +00

ILowor corr BOC MI

Bnnhed = 35

- kpethd :...

$.108580 E+01, .109858 E+01, .182327 E+01$, . $107110 E+01$,

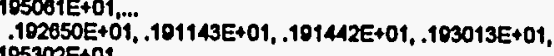
$.189781 E+011, .184410 E+01$, . 189851E+01, .170257E+01, $103855 \mathrm{E}+01$.

$.180571 E+01, .105197 E+01, .103038 E+01, .10774$ AE+01 $151105 E+01, \ldots$

$.154701 E+01, .130703 E+01, .140700 E+01, .120002 E+01$,

$.080282 E+\infty 0, .063091 E+\infty 0, .030532 E+\infty, .778020 E+\infty 0$, $.737582 E+\infty 0, \ldots, 520080 E+\infty 0,409013 E+00,570974 E+00$, $.535207 \mathrm{E}+00$

END

PROCI:D L7

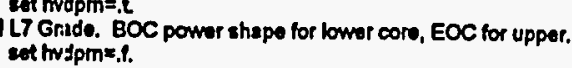

IUpper core EOC. L7

s kpusor $=1.48$ i EOC Upper Core relative power - Knniter $=230$ 
- kpahcu $=$...

$1.20,1.32,1.38,1.45,1.51 \ldots$

$1.51,1.54,1.58,1.04,1.07, \ldots$

$1.00,1.57,1.40,1.43,1.26, \ldots$

$1.10,1.06,1.03$

1 Lower core 80C. L7
8 kploor $=1.701$ i $17 \mathrm{C}$ Lower Cone relative power

knnhel $=23$

trothe =

$2.31,2.29,2.28,2.27,2.25, \ldots$

$2.24,2.21,2.18,2.12,2.06, \ldots$

$2.02,1.06,1.80,9.74,1.01 \ldots$

END $1.39,1.29,1.12,1.04,0.00, \ldots$

PROCED LTNS

cot twdpm $=. t$

5-hode L7 G Giade. BOC power ehapo for lower core, EOC for upper.

sot hudpm=.1.

1 Upper core EOC. L7

- kpucoo $=1.49 \mid$ EOC Upper Core relathe pown

trpentu $=50^{\circ}$

- kpeheu = 1.384, 1.580, 1.720, 1.543, 1.133

ILower core BOC. 17

- kploor = 1.701 i $80 \mathrm{C}$ Lower Core relathe power

kpethd $=2.280,2.166,1.004,1.21,0.773$

END

PROCED GRODS

set hydp $m=. t$.

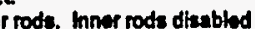

(a)

oseizer = fake.

kneocer $=0.81,0.81,0.81$

END

PROCED HERODS

Rot hwdpm=.L

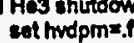

8 kwroct $=0 ., 0 ., 3 ., 3$.

stroct(22) $=1.03,1.03,1.03$

END

PROCED HROOS

cel hudpm=t

I Hydraulic outer rods (20m, IInch, PO=2MPa, TravesOme)

set thudpm=.

sasoct $=5,25,25,0,0.01,10$

END
PROCED P335

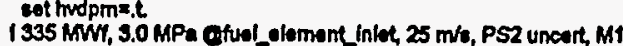

grade

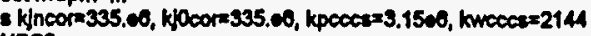

UPS2

PROCED RELAP

IRELAP5 Bonchmank Paramotors for 10 101 (See RELAP2 1002) set hodpmef.

In RELAP

IB Grading used

shucor $=1.14$

I Hot stroak uncartalnty

Shaheu = 1.305, 1.217, 1.204, 1.243, 1.312 I Hot spot une. uppor

core thel $1.394,1,400,1,374,1,352,1.2431$ Hot epot une. lower

core

8 kjnoor $=350.00$

Core lission power $W(W$
core fisston power ( $W$ )

kfacor $=0.0692247$

kdecor $=0.00240$
kercor $=2.00-0$

kerves $=2,000$

ikmoces $\approx 2102.0$

- Kkccll $=1.0002$

- kketfl $=0$.

kglgac $=0.025$

ketihl $=1.2$

- ketpel = 1 .

cecumulatior

- ketmel $=1$.

accumulator

ccumulatox

$\log _{20.0900}=3.7$

END

PROCED RELAP2

IRELAPS Benchmank Parametare for 1092 (Clowe to CDR config)

set hudpminch

For RELAP banchmark only. WRONG :III

I Match RELAP P Q accumulator

Match RELAPP

IMatch RELAPP 0

I Makch RELAP P o accumulator

UT LT in RELAP

Uincor $=320.00$

in kincor = 330.06

kjocor $=343.6600$
$k d \theta c o r=0.002480$

8 keroor $=0.140 .6$

$s$ keves $=0.140-0$

- kwoces = 1994.2

8 koweor $=0.0005$

Hwect $=15.0$

127 Grading

$105 \%$ uncortainties

al power (i) (104.2\%)

Avg eq diam UC LC (m)

C.W off roughness $(m)$

Total priman fiow (kofs)

ITo agree with REL AP fow splits

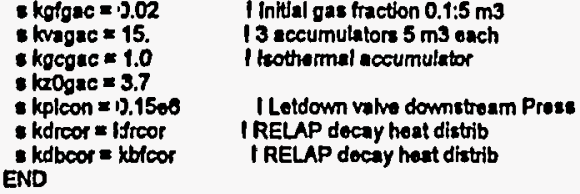

PROCEO RELAPS

IRELAP5 Banchmark Parametere for 10922 (200 MW (104.2X), 17.0

eot modpm*t.

RELAP2

Nincor $=200.00 \quad$ I Core nominal fission power

i. kjocor $=208.400$

K Kweces = 1336 .

Total primary flow (kg/s)

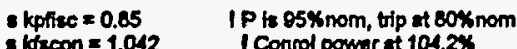

ifficon = $=1.042 \quad$ Conrol power at 104.2\%

PROCED RELAPA

IRELAPS Bunchmark Parameters for 1002 (Almost CDR config)

RELAP2

1 Intitial gas fraction 0.52:7.52 $\mathrm{m3}$

skercor = $1.13 \quad$ I Forces agreement with RELAP P-out

kpfice = $1.05 \quad$ IP is 85\%nom, tip at 80\%

Ifreon $=1.042$

i connol power at 104.2\%

proced RNC

IRELAP5 Benchmark - Nat Cire (10192)

eet thdprne.?.

This ka strango (very) station blackout. MI undorztund the RELAP dat corroctly. At tmo=0 they lolate the coconda but the primary pumps koep running untill ecrem Ialso, socondary side natural circulation fis diasbled (they lastume botal holation

The letdown valve ts also assumed fully open

IThis mecro does not really do all that H makes some appron

ection 'clear' 


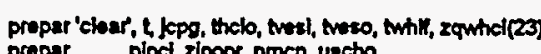

prepar Plpct, zlpopt, nmep, uncto

propar
propar

RELAP3 $1200 \mathrm{mw}, 17 \mathrm{~m} / \mathrm{s}$ core

- KtemcP $=3$. ITY to malch RELAP costionn - Knplece a

s ktloon $=0$. I Keep lettown value at current postition

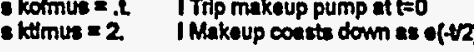

- kkttoon $=0$. ITip recondary coolant pump

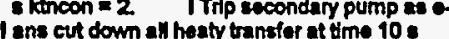

ectlon var=10, war=0, toc'zkeambs

is kdhthi $=0.1$

s kdhted = $=0.1$ bollmil nat cire in sece (ieolation)

- tatop $=100$

cint $=1$.

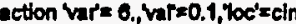

action vareso., var=10., toc'xecting

1 Modifiod er21/103 b fudge old pump tip with scram function

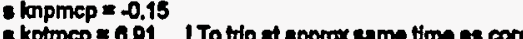

start

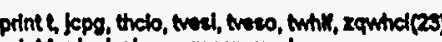

print, plpci, zlpopp, nmep, uscto

printe castel(23) cthe(p3)

prith i, zquhel(23), zquhc|(23), cartcl(23)

and

PROCED RBOC

IRELAPS Benchmark - 00 CENT REACTIVTY STEP sol twdprm=.1.

ection 'cloar
RELAPA

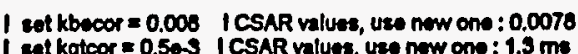
ent kartra(1) $\approx 0 ., 0.8,0.8$

cot kartre $(11)=0 ., 0.001,1.010$

propar "clear, 4 fentr, jacho, jeps, mathet, metted propar xier, rike, xocr, roct

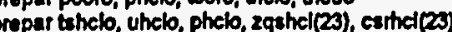

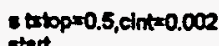

print t fente jectio, kppg, mashet, methed

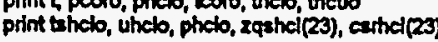

END

PROCED RTD

IRELAPS Benctrnark - C 14inch OEG brosk 1.1 \& (11/02)

set hudprn=.1.

ection iclear

- kbdell = kdect 114 inches 10

$8 \mathrm{kgbcll}=$, true. IDEG braak (doubles flow)

- ktell = 1.1

prepar cloar, 2 pipcl, zipopr, meto, welic, pdet

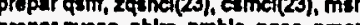

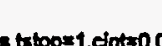

otat

print t, plpel, zlpopr, mero, weile, pdet

(2), mithed, methed

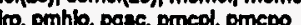

END

PROCED R2B

IRELAPS 10\%1Bonchmark, 2 inch, sharp odgo, 250 ma CPBT broak

Rethentos.

RELAP1

\begin{tabular}{|c|c|}
\hline 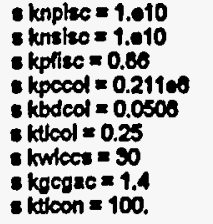 & 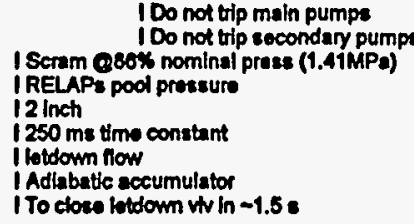 \\
\hline \multicolumn{2}{|c|}{ 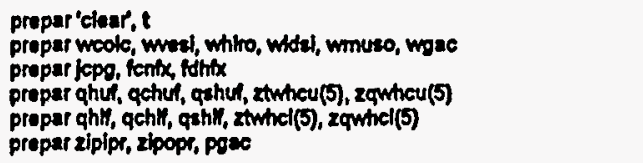 } \\
\hline
\end{tabular}

ection var =5., vare 60, loc'swmuse Intandby pump sint $=0.0002$

print t, waxte, unesl, whilo, whdsi, wnuzo, wgse

print L, jeppl, fenter foth

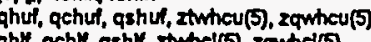

print 2 ziphix, zipopr, pgece

END

PROCED SSPAR

Parmmeters fudgod to agree with Gradya SS code

(Fet by hand the Inlet outbot pressure (KPCCCS=2.7TEO)

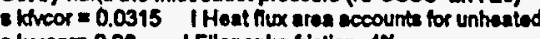

END

Procod CSARF

Intel conditions

(includes masururomentroperational uncen

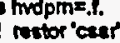

: kjocor $=343.09200$ i Set hitial powner at 104.24\%

- Wweces $=2122.50$ i Sol intial frow at oox

Itecon = 45.6 I Control somperature it 101.41\%

I Parameters to agreo with s.s. code consenative essumptions

kpeces $=2.7706$ 1 To ent Pdnlot $=285$

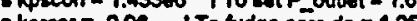

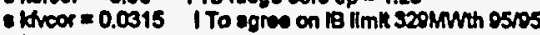

procod CSARL

Indpm:.t

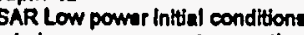

(includes nreasurement+operational uncorti)

1 metor 'cear

- kjocor $=23.03$ I Sot initial powor at 1.04

kwoces $\approx 2122.56$ is Ist intitial flow at $90 \%$

B Ktecon $=1.15 .6 \quad$ Control tomponature at 101.41\%

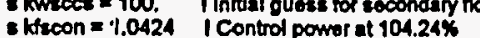

Parameters to agroe with 8.s. codo conzonative assumptions

8 kpcccs $=2.7706$ 1To eot P.inlet $=2.85$

- kpecon $=1.43300$ I To set P_outtel $=1.01$

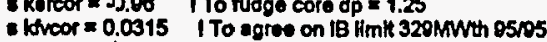

procod Nowrod

I Rod worthe wot bo 1 dolar por $50 \mathrm{~mm}$, max $-10 \mathrm{dol}$

i Assume. $50 \mathrm{~mm}$ to Insert 1 dol consorvativo to account for 1.rod fallure

Iectual 3-rod numbor vary from $22.3 \mathrm{~mm}$ to $30.3 \mathrm{~mm}$

Assumod criticality at contertho, and hoarth 
D-8

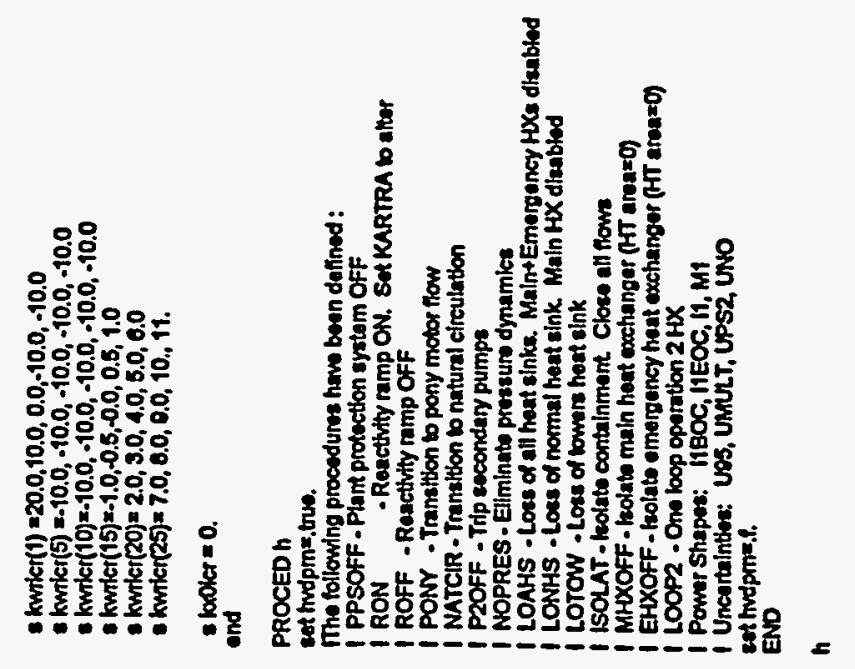


Appendix E

NOMINAL ADVANCED NEUTRON SOURCE OPERATING CONDITIONS PREDICTED BY THE ADVANCED NEUTRON SOURCE DYNAMIC MODEL 

APPENDIX E. NOMINAL ANS OPERATING CONDITIONS PREDICTED BY ANSDM

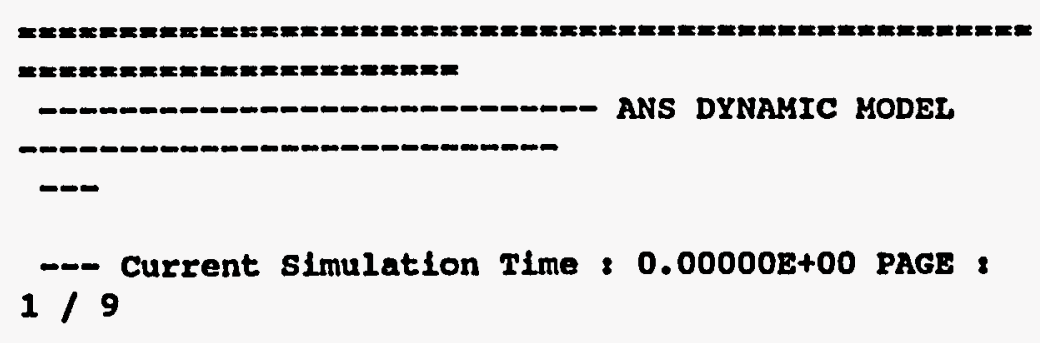

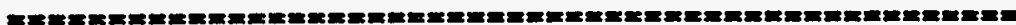

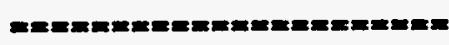

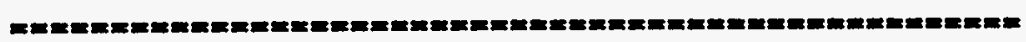

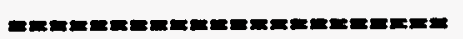

-

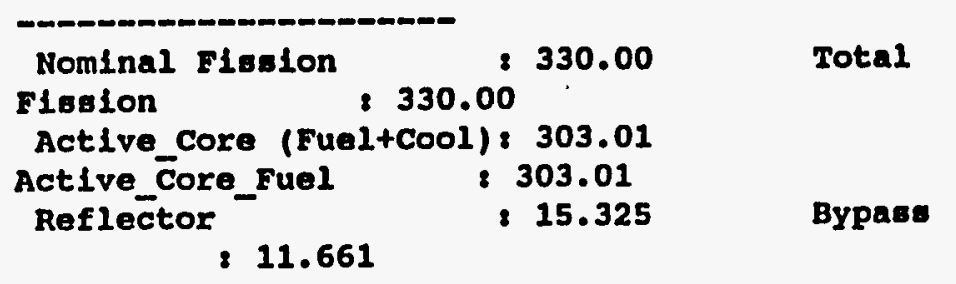

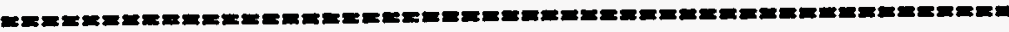

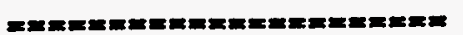

-

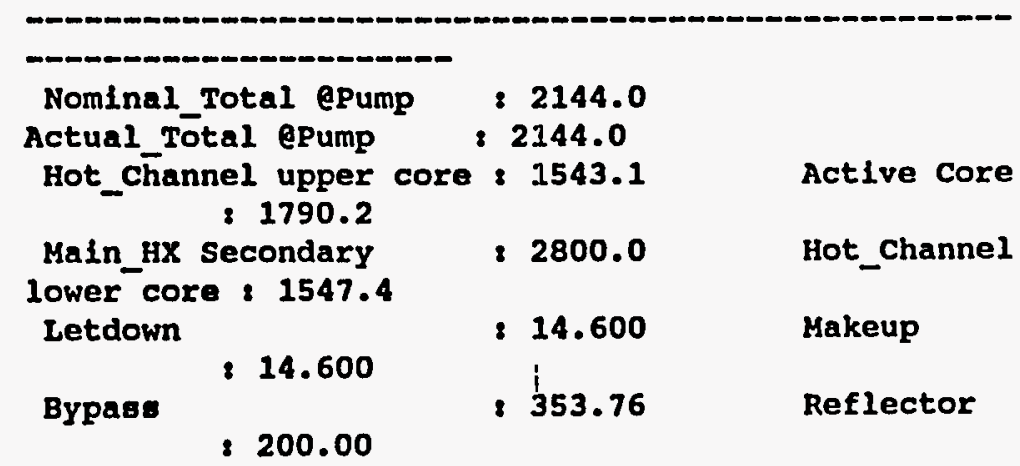

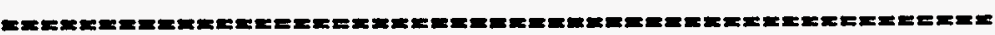

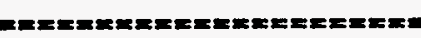

- -

\begin{tabular}{|c|c|c|}
\hline $\begin{array}{l}\text { Core Inlet } \\
\quad 1.8103\end{array}$ & 3.1991 & Core Outlet \\
\hline $\begin{array}{l}\text { Pressurlzer } \\
\text { Letdown/Makup }\end{array}$ & $\begin{array}{r}1.5864 \\
1.6904\end{array}$ & \\
\hline $\begin{array}{l}\text { Maln_HX Primary Inlet } \\
\text { Primary Outlet : } 1.6702\end{array}$ & 1.6904 & MaIn_HX \\
\hline $\begin{array}{l}\text { Pump Inlet } \\
3.2931\end{array}$ & 81.6513 & Pump Outlet \\
\hline
\end{tabular}

Maln HX Secondary Inlet:0.26209 


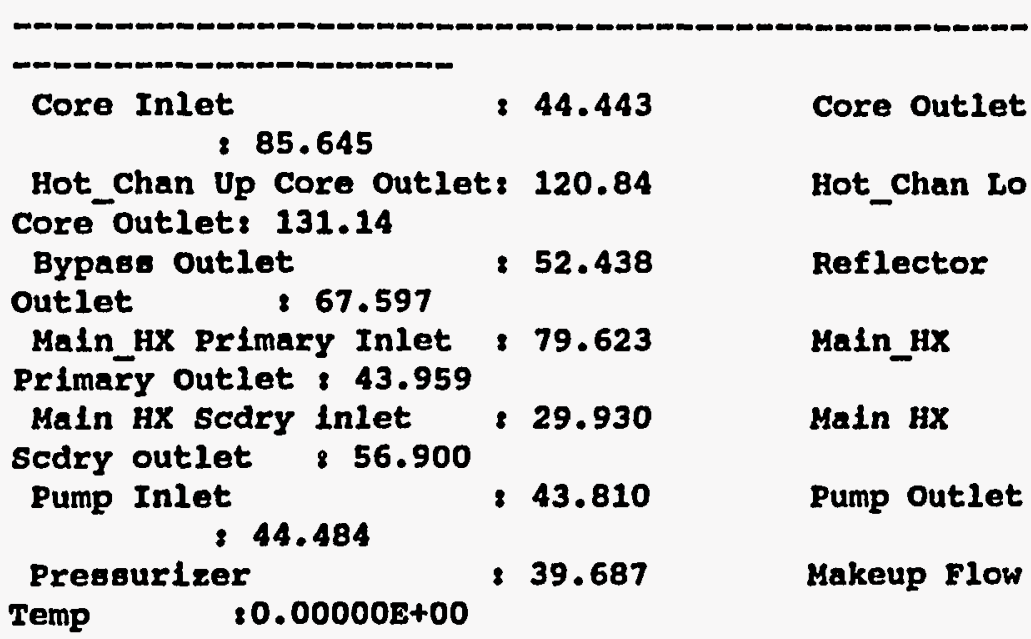

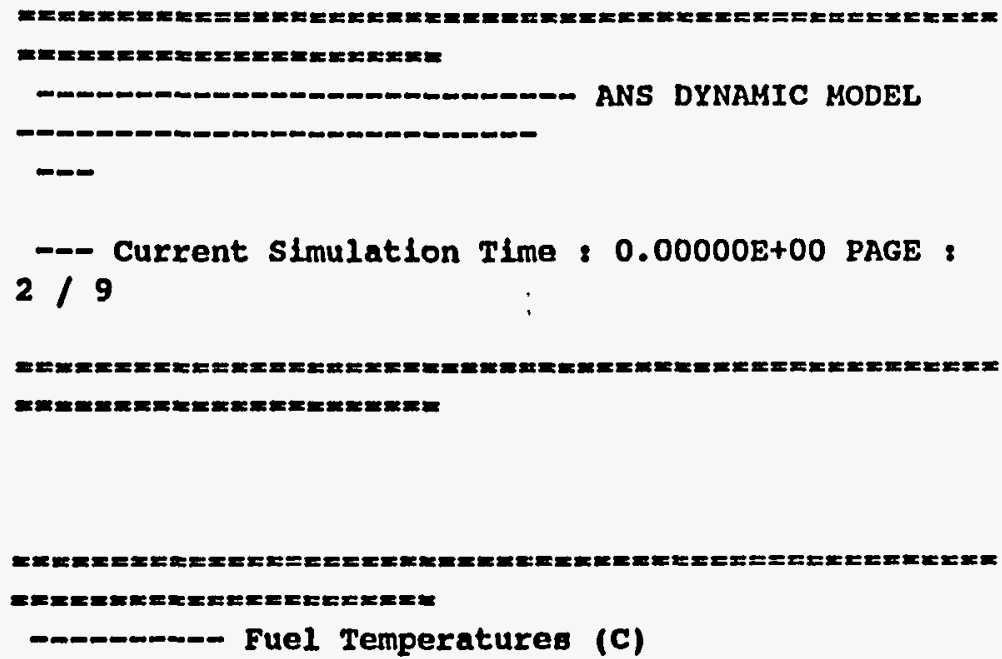

Avg_Channel Actual $\quad$ : 5.7096 Avg_Channel


Hot_Chan Up Core Actual: 9.1369

Core Crit. : 28.004

Hot_Chan Lo Core Actual: 10.425

Core Crit. \& 26.277

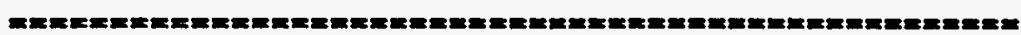

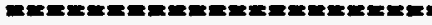

-mmmon- Critical Heat Flux Ratios

\begin{tabular}{|c|c|c|}
\hline $\begin{array}{l}\text { MIn CFR Upper } \\
\text { Lower Core } \\
\text { Location CFR }\end{array}$ & $\begin{array}{l}\text { Core } 80.10000 \mathrm{E}+11 \\
: 0.10000 \mathrm{E}+11 \\
\text { Upper Core } 0.10000 \mathrm{E}+11\end{array}$ & Location \\
\hline $\begin{array}{l}\text { CFR Lower Core } \\
\text { Min CSR Upper } \\
\text { Lower Core }\end{array}$ & $\begin{array}{l}20.10000 \mathrm{E}+11 \\
\text { Core } \quad 0.10000 \mathrm{E}+11 \\
80.10000 \mathrm{E}+11\end{array}$ & Min CSR \\
\hline $\begin{array}{l}\text { Min IBLR Uppe } \\
\text { Lower Core }\end{array}$ & $\begin{array}{l}\text { Core } 10.55555 \mathrm{E}+34 \\
0.55555 \mathrm{E}+34\end{array}$ & MIn IBLR \\
\hline
\end{tabular}

作

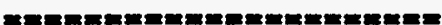

- nond Verel Inlet (Cold Leg) Coolant

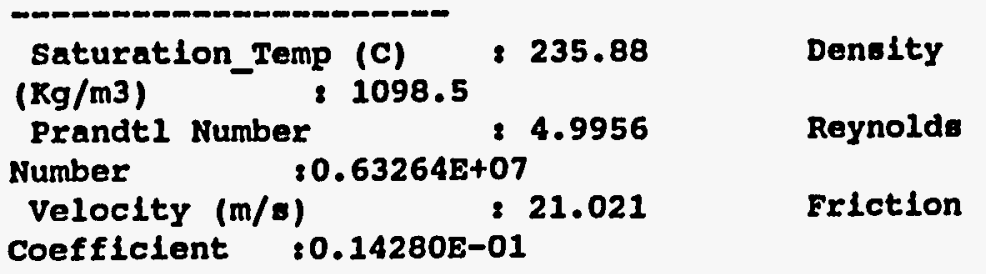

Hot_Chan Up

Hot Chan Lo

Avg_Ch Outlet Coolant Properties

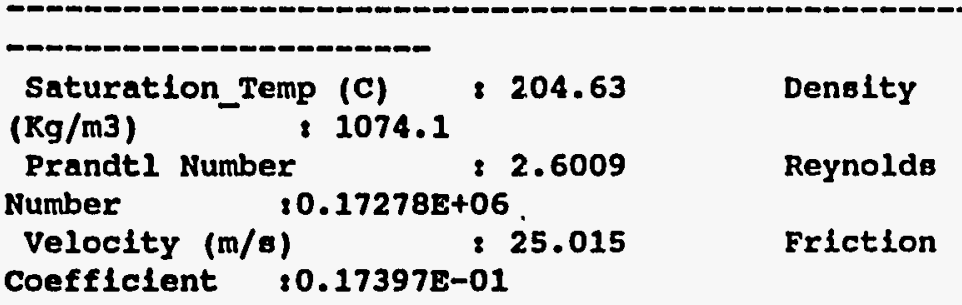

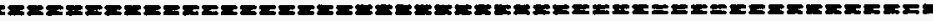

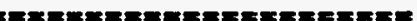

- - Hot_Ch Upper Core Outlet Coolant Prop
Density

Reynolds

Friction
Velocity $(\mathrm{m} / \mathrm{s})$

Coefficlent
$0.21415 \mathrm{E}+06$

24.603

$: 0.16672 \mathrm{E}-01$ 

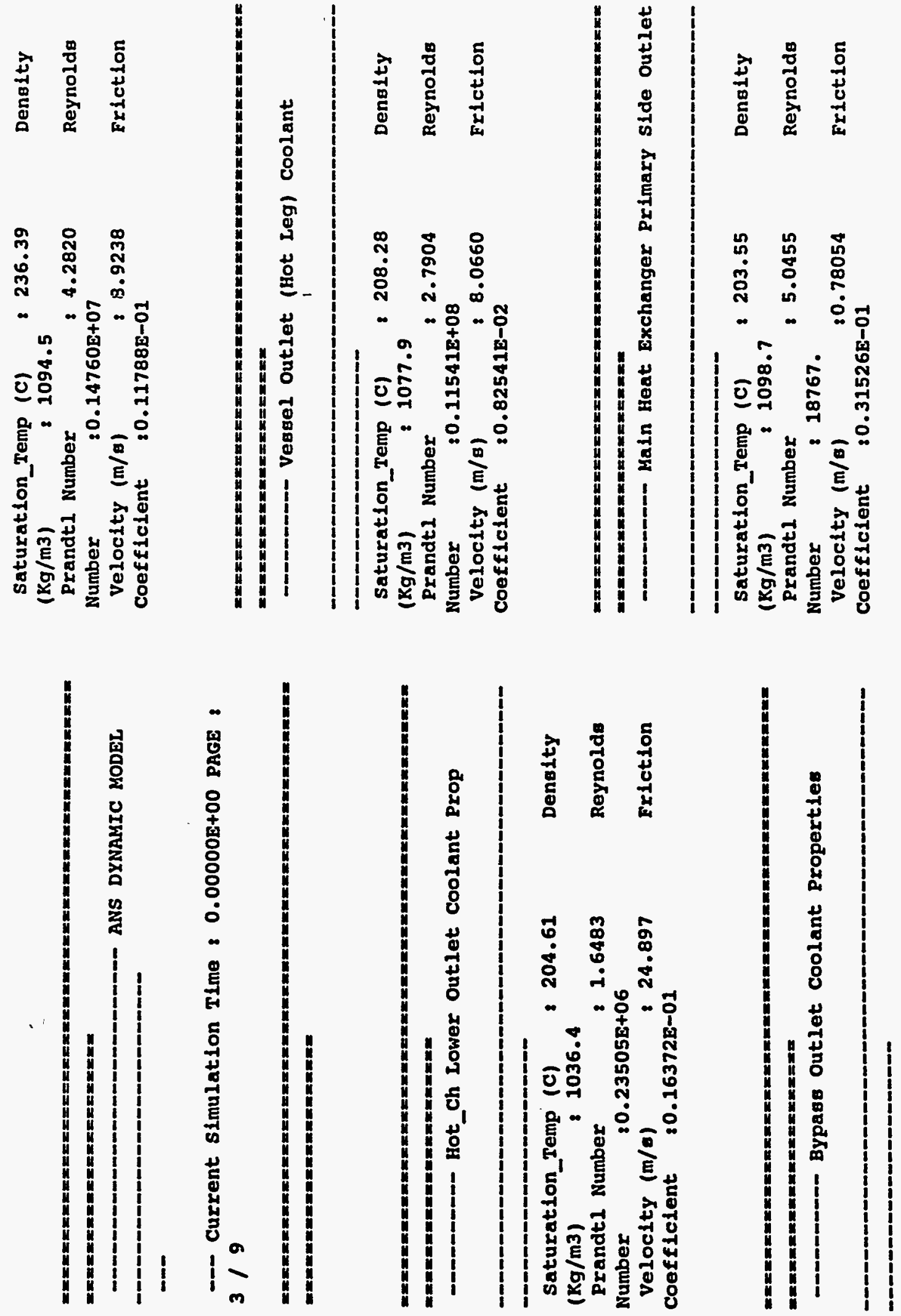


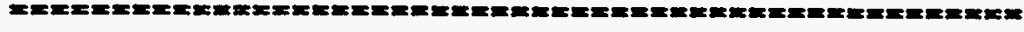

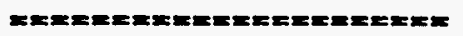

outlet

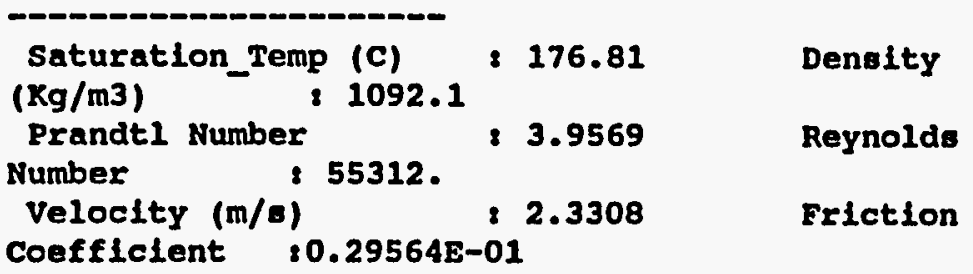

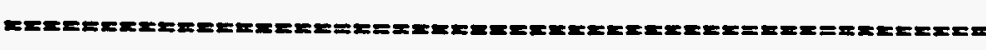

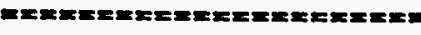

ANS DYNAMIC MODEL

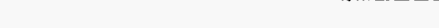

- Current simulation TIme : 0.00000E+00 PAGE :

$4 / 9$

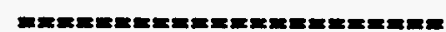

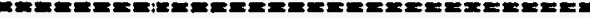

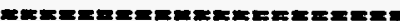

Core Properties

Actlve Fuel Volume (m3):0.33700E-01

Act Coolant

Volume $(\mathrm{m} 3): 0.33700 \mathrm{E}-01$

Active Fuel Height $(\mathrm{m}), 0.52700$

Fuel

Thlckness (m)

$0.12700 \mathrm{E}-02$

Fuel Density $(\mathrm{kg} / \mathrm{m} 3)$ \& 3870.0

Capacity $(\mathrm{J} / \mathrm{Kg} / \mathrm{K}) \quad 0.52700$

Ef Ht Tre Coef $(\mathrm{w} / \mathrm{K} / \mathrm{m} 2) \geq 0.00000 \mathrm{E}+00$

Area ( $\mathrm{m} 2$ ) :0.66630E-01

Ch. Equiv. Diameter (m) $: 0.25400 \mathrm{E}-02$

Dlameter $(m): 0.00000 \mathrm{E}+00$

LoC Press Loss ( $V$ head) \& 1.0000

Flow Length $(\mathrm{m}): 0 . \overline{5} 2700$

Bypass Volume (m3)

$20.33700 \mathrm{E}-01$

Area (m2) $\quad 0.36220 \mathrm{E}-01$
Heat

Core Flow

Hot Ch Eq.

Channel
Bypase Flow 


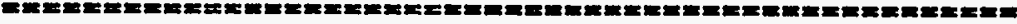
|

-non- Core Neutronlce

Core react coeff $\$ / \mathrm{dr} / \mathrm{x} \mathbf{2} 7.4000$

coef $\$ / d x / x \& 9.1000$

Fuel Doppler coef $(\$ / K) \geq-.12500 \mathrm{E}-02$

coef $\$ / \mathrm{d} x / \mathrm{x} 2 \mathrm{29.000}$

Eff Beta (delayed neut) : 0.77400E-02

Generation TIme $(8): 0.50000 \mathrm{E}-03$

- Delayed Neutron Fractions

$0.42748 E-03 \quad 0.14662 E-02 \quad 0.13113 E-02 \quad 0.33248 E-02$ $0.77441 E-03 \quad 0.27879 E-03$

-- Delayed Neutron Time Constants $(8-1)$

0.14300E-01 0.30500E-01 0.11100 0.29600

1.1300

3.0000

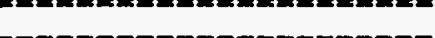

- - m - Decay Heat Model

Fraction In Bypase (8) 826.360
Reflector 8.8 .6400 $\begin{array}{ll}\text { Fraction } \\ \text { Time }(8)=0.10000 \text {-09 Decay Heat }(8)=5.7544 \\ \text { Time }(8)=1.0000 & \text { Decay Heat }(8)=5.7544 \\ \text { Time }(8)=10.000 & \text { Decay Heat }(8)=4.7863\end{array}$

Bypass reac

Reflec reac

Ef
Decay Heat $(s)=4.7863$
Time $(8)=100.00 \quad$ Decay Heat $(8)=3.2359$

Time $(B)=1000.0$ Decay Heat $(8)=1.7783$

Time $(B)=10000$. Decay Heat $(8)=0.87096$

Time $(8)=0.10000 \mathrm{E}+06$ Decay Heat $(8)=0.30200$

TIme $(\theta)=0.10000 E+07$ Decay Heat $(\xi)=0.10233$ 


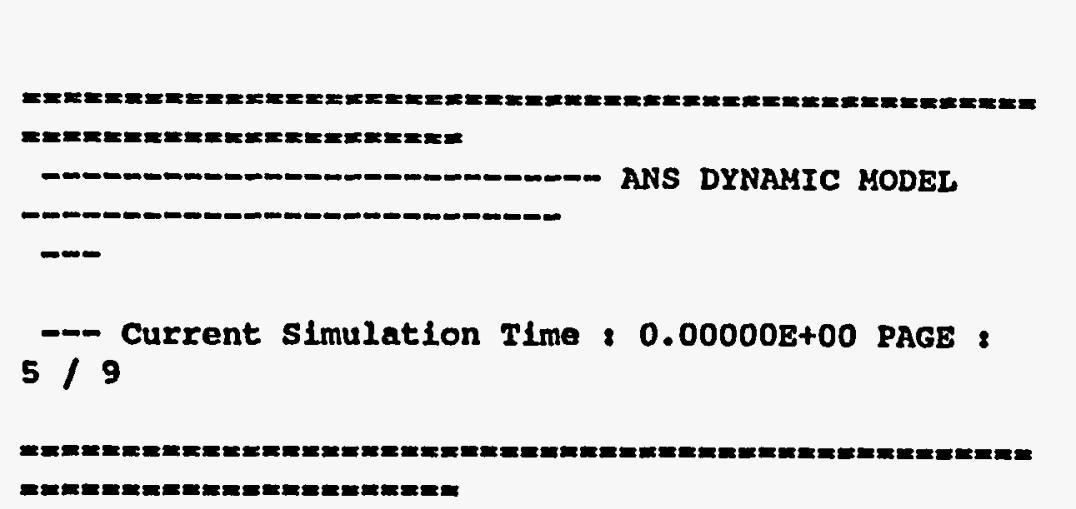

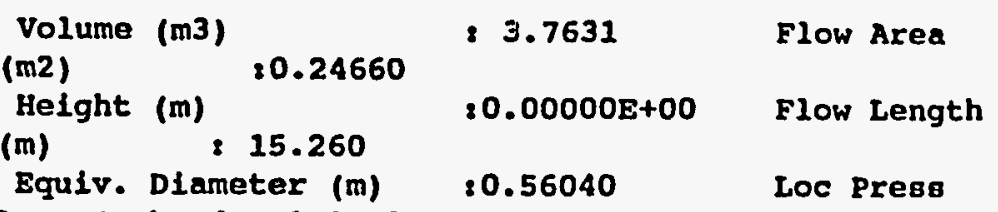

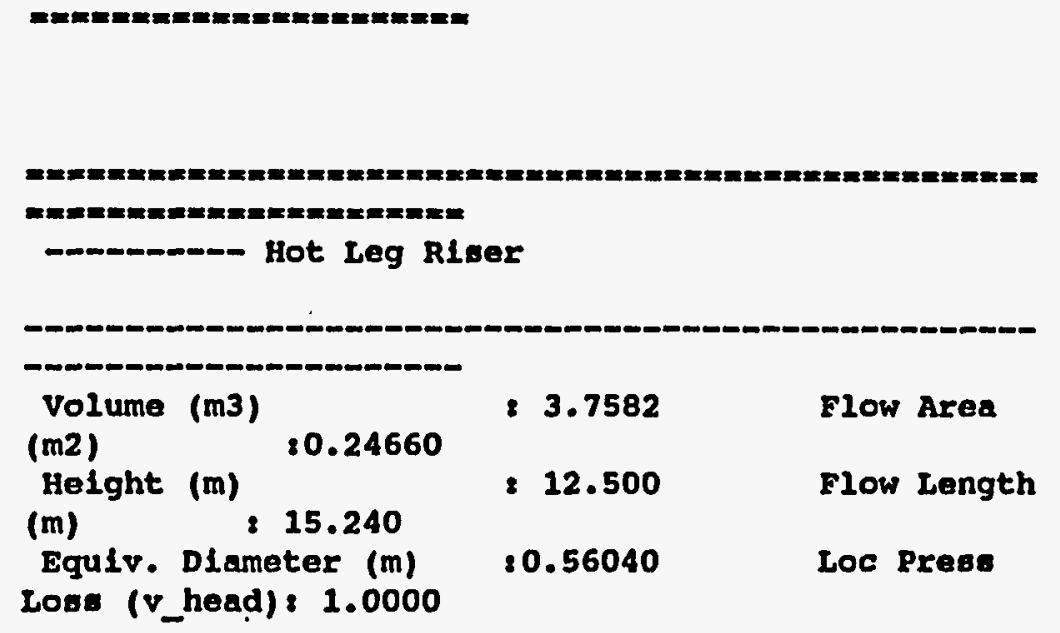

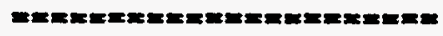

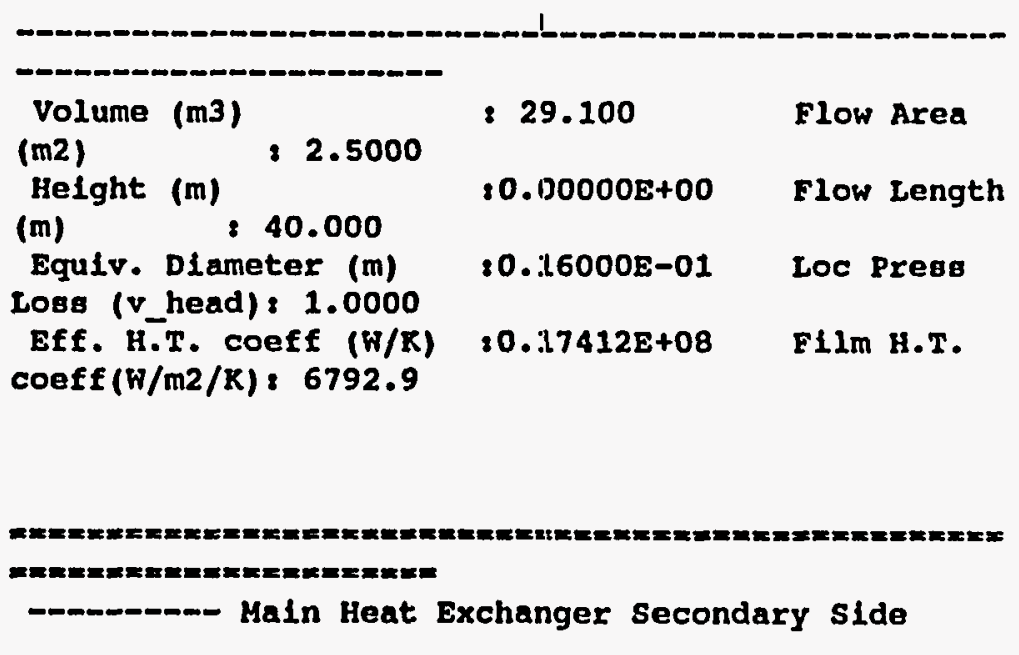

$\begin{array}{lll}\begin{array}{l}\text { Volume }(\mathrm{m} 3) \\ \text { (m2) } \\ \text { Helght }(\mathrm{m}): 1.1000\end{array} & : 29.100 & \text { Flow Area } \\ \begin{array}{l}\text { (m) } \\ \text { Equlv. Diameter }(\mathrm{m})\end{array} & : 0.12700 \mathrm{E}-01 & \text { Loc Press }\end{array}$


Loss ( $v$ head) 21.0000

Total foul. res $(\mathrm{m} 2 \mathrm{~K} / \mathrm{W}): 0.26000 \mathrm{E}-03$ Film H.T. $\operatorname{coeff}(\mathrm{W} / \mathrm{m} 2 / \mathrm{K}): 10493$.

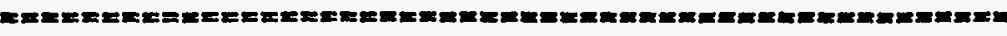

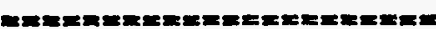

namon- Cold Leg (Horizontal Run)

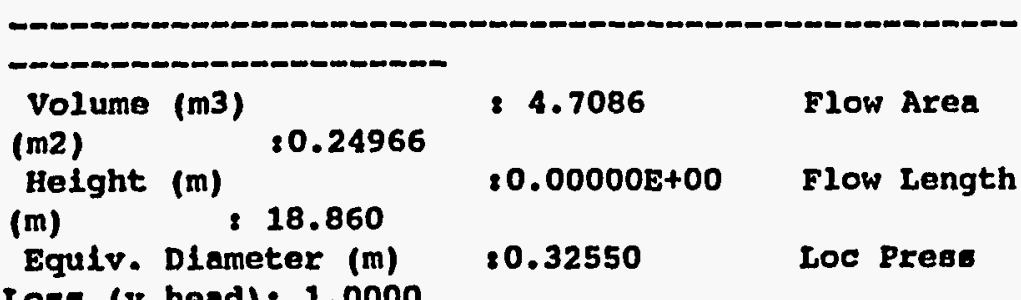

Lors (v head) 81.0000

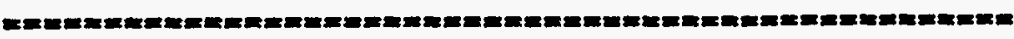

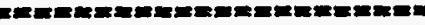

- nommon Cold Leg Rleer

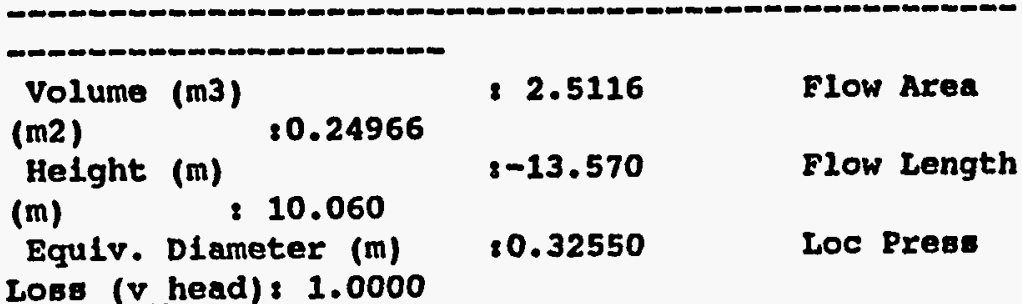

LOBB ( $v$ head) $: 1.0000$

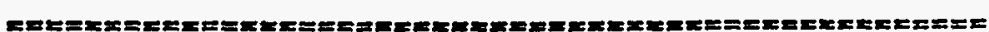

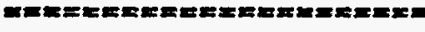

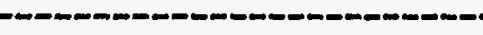

ANS DYNAMIC MODEL

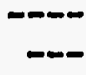

- current simulation TIne :0.00000E+00 PAGE : $6 / 9$

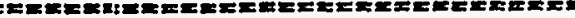

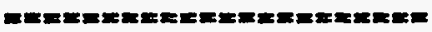

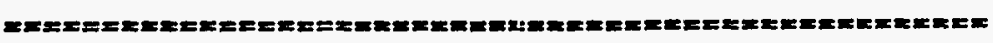

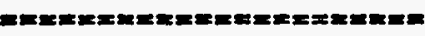

- - -

$\mathrm{RX}$ Pool volume $(\mathrm{m} 3) \quad 500.00$ Heat load

(MW) $\quad: 0.12345 E+07$

Plpe Chase Pool Vol (m3): 300.00 Heat load

(MW) $\quad: 0.16143 \mathrm{E}+07$

HX Pool volume $(\mathrm{m} 3)$ \& 1200.0 Heat load

(MW) $\quad 80.21258 \mathrm{E}+07$ 


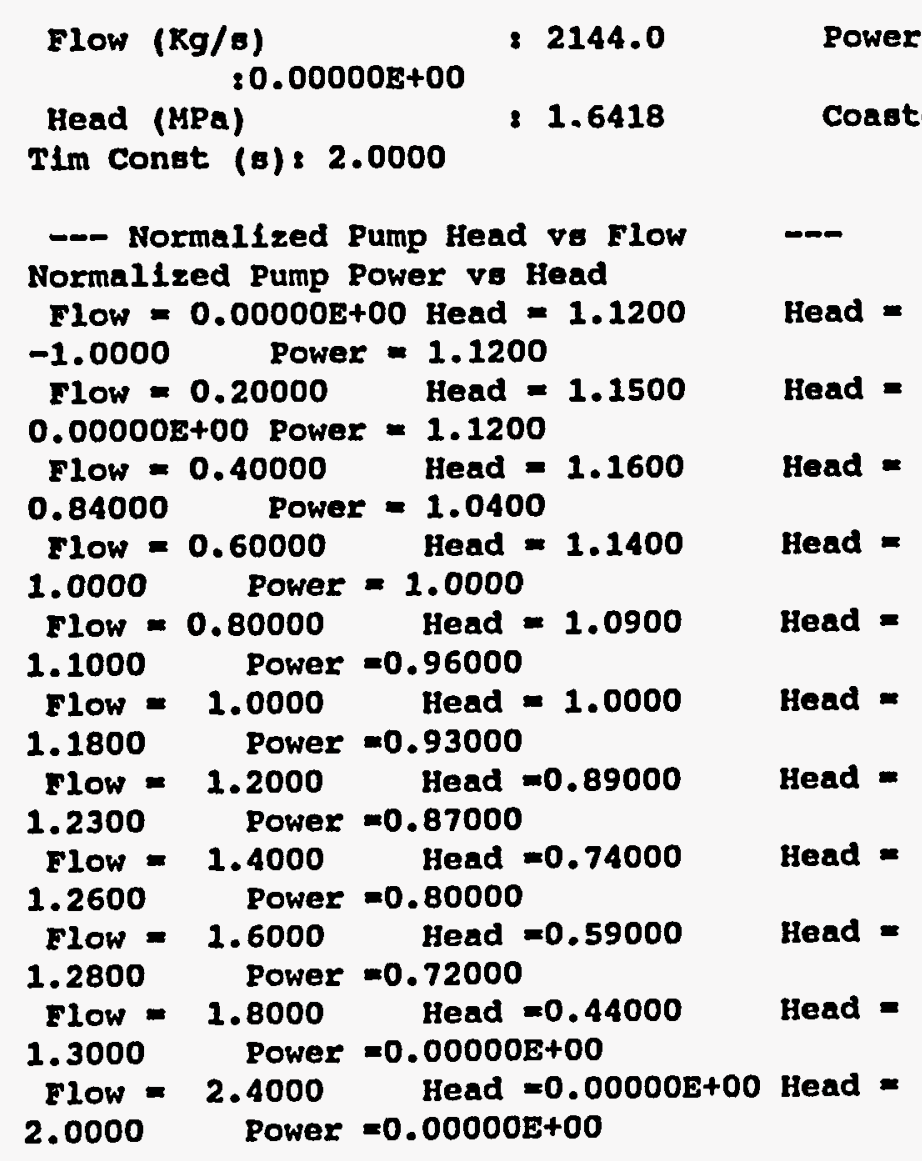




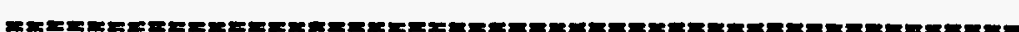

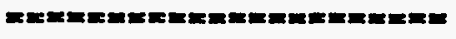

DYNAMIC MODEL

$-\infty$

- Current simulation Time $\& 0.00000 E+00$ PAGE :

$7 / 9$

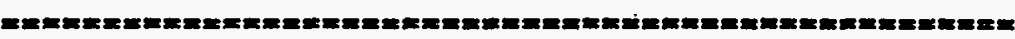

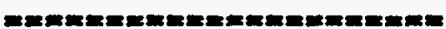

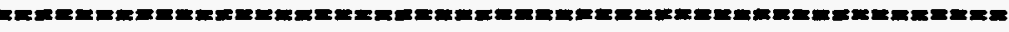

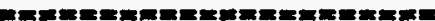

- - Reflector Region

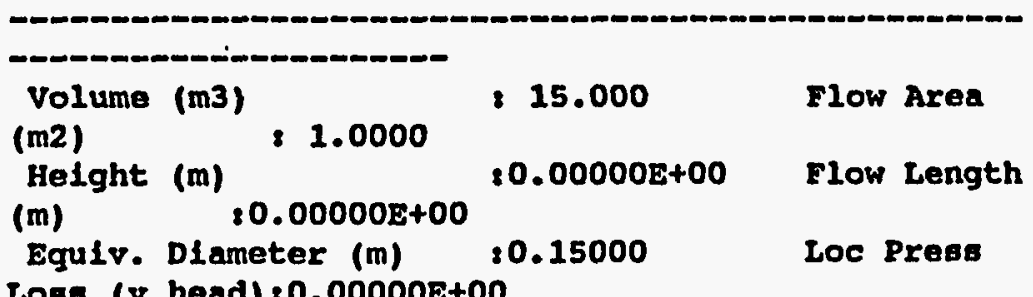

Lose ( $v$ head) $80.00000 E+00$

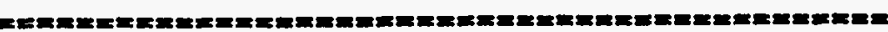

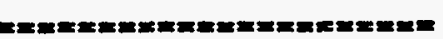

- - - Gas Accumulator
Total Volume (m3) \&21.000 Gas Volume

Fraction $\quad 20.74000 \mathrm{E}-01$

Level (m) \&4.6300

Rate ( $\mathrm{kg} / \mathrm{s}) \quad: 0.00000 \mathrm{E}+00$

Outlet Pipe Length $(\mathrm{m})=5.0000$

Mass Flow

Flow Area $(\mathrm{m} 2): 0.24966$

Equiv. Diameter (m)

Loss ( $v$ head): 1.0000

Effective $L / A$

20.32550

otlt Plpe

Loc PresB

10.000
(1)

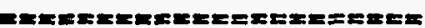

- - Leak Effective Dlameters (m)

- - -

$\begin{array}{ccc}\text { ecore Inlet } & : 0.00000 \mathrm{E}+00 \text { ecore } \\ \text { outlet } & 0.00000 \mathrm{E}+00\end{array}$




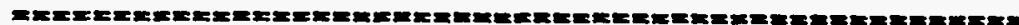

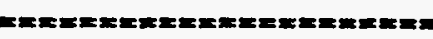

- ANS DYNAMTC MODEI

-- Current simulation Time : 0.00000E+00 PAGE : $8 / 9$

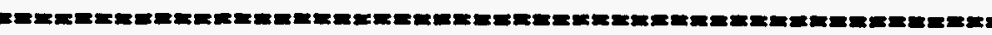

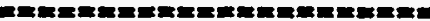

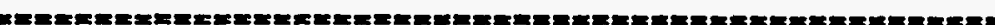

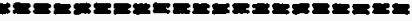

Friction Pressure Drops (MPa)

Across Active Core
HX $0.26379 \mathrm{E}-01$

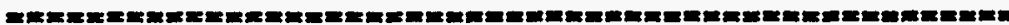

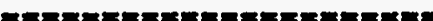

Inner Control Rod scram Syatem

\footnotetext{
Flux/Flow setpolnt (8) $: 115.00$ setpolnt 80.20000
}

Flow setpoint (*), 8.0000

Flux rate
Setpolnt (MPa): 1.3584

Inlet Temp setpoint (C): 55.000

Scram Delay Time (s) $20.30000 \mathrm{E}-01$

Init C Rod Position (m):-.15000 Max. C_Rod Insertion $(\mathrm{m}): 0.60000$

- Control Rod Acceleration ve Position $z=0$ $\Rightarrow$ Top of Active Core)
$z=0.00 \mathrm{~m}$,
accel $=58.860$
accel $=9.8100$
$\mathrm{m} / \mathrm{s}$
$2=0.15 \mathrm{~m}$,
accel $=9.8100$
$\mathrm{m} / \mathrm{s}$
$z=1.00 \mathrm{~m}$,

-- Control Rod horth ve Position $(z=0 \Rightarrow$ Top of Active Corel

$\begin{array}{lll}z=0.00 \mathrm{~m}, & \text { worth }=9.1900 & \$ \\ z=0.10 \mathrm{~m}, & \text { worth }=9.1900 & \$ \\ z=0.20 \mathrm{~m}, & \text { worth }=7.7300 & \$ \\ z=0.30 \mathrm{~m}, & \text { worth }=6.6100 & \$ \\ z=0.40 \mathrm{~m}, & \text { worth }=3.6600 & \$ \\ z=0.60 \mathrm{~m}, & \text { worth }=2.1400 & \$ \\ z=1.00 \mathrm{~m}, & \text { worth }=-.18000 & \$ \\ z=0.00 \mathrm{~m}, & \text { worth }=-1.5000 & \$ \\ z=0.00 \mathrm{~m}, & \text { worth }=-4.9500 & \$ \\ z=-0.25 \mathrm{~m}, & \text { worth }=-8.1500 & \$ \\ z=-2.02 \mathrm{~m}, & \text { worth }=-11.580 & \$ \\ z=-3.11 \mathrm{~m}, & \text { worth }=-14.030 & \$ \\ z=-4.00 \mathrm{~m}, & \text { worth }=-15.210 & \$ \\ z=-4.60 \mathrm{~m}, & \text { worth }=-15.210 & \$ \\ z=-6.58 \mathrm{~m}, & \text { worth }=-1.0000 & \$ \\ z=-8.53 \mathrm{~m}, & \text { worth }=-.60000 & \$ \\ z=-8.53 \mathrm{~m}, & \text { worth }=-.50000 & \$ \\ z=-1.00 \mathrm{~m}, & \text { worth }=-.40000 & \$ \\ z=-0.80 \mathrm{~m}, & \text { worth }=-.30000 & \$ \\ z=-0.60 \mathrm{~m}, & \text { worth }=-.20000 & \$ \\ z=-0.50 \mathrm{~m}, & \text { worth }=-.10000 & \$\end{array}$




\section{E-14}

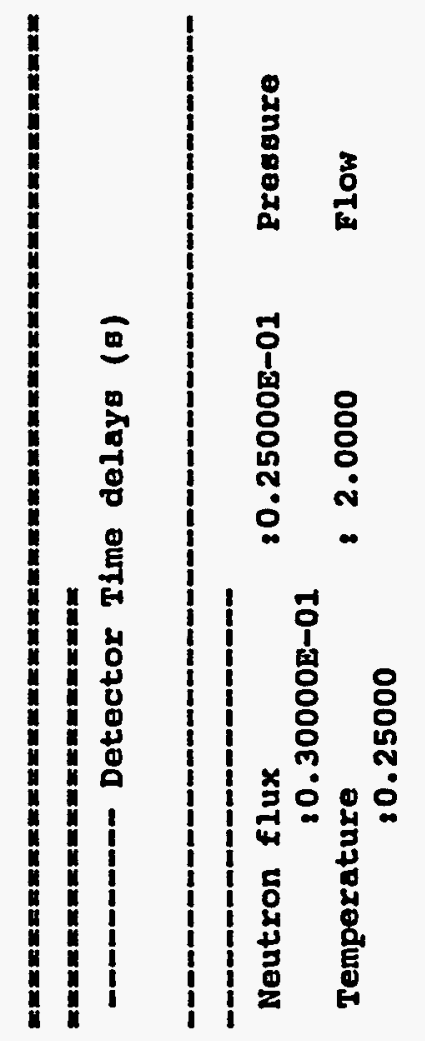


Appendix F

IIST OF ALL ADVANCED NEUTRON SOURCE DYNAMIC MODEL VARUABLES AND THEIR NOMINAL VALUES 


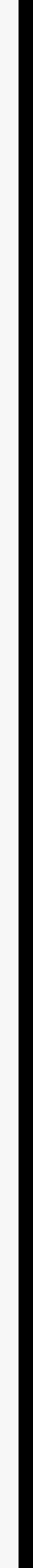




\section{APPENDIX F. LIST OF ALL ANSDM MODEL VARIABLES AND THEIR NOMINAL VALUES}

State Variableg Initial Conditions DNDET 0 .

ZDNDET 0 . ECPG 0 .

ZIECPG 0 .

FCNFX 1.00000000

ZINCNT 1.00000000 FDET 1.00000000

ZIFDET 1.00000000 HACHO 357191.000

ZIHACC 357191.000 HBYPO 220896.000

ZIHBYP 220896.000 HCLRI 187863.000

ZIHMCL 187863.000 HCLRO 187783.000

ZIHCLR 187783.000 HCORO 357191.000

ZIHOPR 357191.000 HCTBO 127200.000

ZIHCTB 127200.000 HEHXO 185213.000

ZIHEPP 185213.000 HESHL 155891.000

ZIHESP 155891.000 HHCLO 545474.000

ZIHHLC 545474.000 HHCUO 502162.000

ZIHHUC 502162.000 HHLRO 334207.000

ZIHHLR 334207.000 HHXPO 148200.000

ZIHHXP 148200.000
Derivatives

DDNDET 0.

JCPG $3.3000 E+08$

DNCNT-2 - 7057E-07

DEDET 0.

ZDDACC 150.256000

ZDDBYP 14.2309000

ZDDMCL-0.40749600

ZDDCLR-0.50672300

ZDDOPR 0 .

ZDDCTB-1.2745E-06

ZDDEPP 23.7187000

ZDDESP 0.00466240

ZDDHLC 2699.44000

ZDDHUC 238.107000

ZDDHLR-0. 46265300

ZDDHXP-0.71966300
HIPCI 187933.000 ZIHIPR 187933.000

HLHLO 333542.000

ZIHIHL 333542.000 HMCPI 185153.000

ZIHPCL 185153.000 HMFLO 333929.000

ZIHMHL 333929.000 HMHXO 185769.000 ZIHMHP 185769.000 HMRPO 148200.000

ZIHMRP 148200.000 HPCHO 148200.000

ZIHPCH 148200.000 HREFO 283287.000

ZIHREF 283287.000 HSHLX 239306.000

ZIHSHP 239306.000 HTCLO 127200.000

ZIHTCL 127200.000 HTHLO 239306.000

ZIHTHL 239306.000 HVESI 187783.000

ZIHIFD 187783.000 IPOI 1.00000000

ZIIPOI 1.00000000 MMCS 0 .

ZIMMCS 0 PCIL 3.4001E+06 ZIPCIL 3.4001E+06 PCOL 1.8414E+06

ZIPCOL $1.8414 \mathrm{E}+06$ PDET 1.6980E+06

ZIPDET 1.6980E+06 PPOI 1.00000000
ZDDIPR-608.837000

ZDDLHL-0.43457300

ZDDPCL-0.00150202

ZDDMHL-0.25996300

ZDDMHP 25.1418000

ZDDMRP 0.00579261

ZDDPCH 0.01194720

ZDDREF-1.5506E-05

ZDDSHP 61.9591000

2DDTCL 0.

ZDDTHL 0 .

ZDDIFD 0.

ZDIPOI-1 . 1830E-12

ZDMMCS 0.06835940

ZDPCIL 0 .

ZDPCOL 0 .

DPDET 8125.00000

ZDPPOI $8.2641 E-15$ 
ZIPPOI 1.00000000 SPOI 1.00000000 ZISPOI 1.00000000 TACF 205.381000 ZITACF 205.381000 TCDET 44.4839000 ZTCDET 44.4839000 THDET 80.0271000 ZTHDET 80.0271000 THLF 296.366000

ITHLF 296.366000 THUF 266.990000

ZITHUF 266.990000 VICR 0 .

ZIVICR 0

VLGAC 19.4460000

ZIVGAC 19.4460000 VOCR 0 .

ZIVOCR 0 . WDET 2144.00000

ZIWDET 2144.00000 WGAC 0.

ZIWGAC 0.

WMCPI 2144.00000

ZIFMCP 2144.00000 WTHLO 2800.00000

ZIWSCC 2800.00000 XICR-0.15000000

ZIXICR-0.15000000 XOCR-0.80000000

ZIXOCR-0.80000000 XPOI 1.00000000

ZIXPOI 1.00000000 z99840 104.000000 ZWECCS 104.000000 2998731.00000000 ZINCON 1.00000000
ZDSPOI $3.1914 \mathrm{E}-13$

ZDTACF 0.10249800

DTCDET-3.2425E-05

DTHDET 0 .

ZDTHLF-0.76695700

ZDTHUF 0.17215000

ZDVICR 0 .

ZDVGAC 0.

ZDVOCR 0.

DWDET -0.27441400

DWGAC 0.

DWMCPI 0 .

ZDWSCC-0.01437500

ZDXICR 0 .

ZDXOCR 0.

2DXPOI 2.4224E-11

299839-0.24569400

299872-8.6018E-04
2DPMCP 1.00000000 2998381.00000000 ZFFGAC 0.

2998420 .

ZFFMUS 0.

z99841 0 .

ZNMCP 1.00000000

ZINMCP 1.00000000

ZPCCNT(1) 59.7868000

ZICCNT(1) 59.7868000

ZPCCNT(2) 96.1457000

ZICCNT(2) 96.1457000

ZPCCNT(3) 23.6277000

ZICCNT (3) 23.6277000

ZPCCNT (4) 22.4650000 ZICCNT (4) 22.4650000

ZPCCNT (5) 1.37064000 ZICCNT (5) 1.37064000

ZPCCNT (6) 0.18585900 ZICCNT (6) 0.18585900

ZPCCNT (7) 2853.59000 ZICCNT (7) 2853.59000 ZPIGAC 0. 2998430.

ZWLCIL 0.

2998270.

ZWLCOL 0 .

2998440.

2XLCON 0.

ZIXCON 0 .

\author{
Algebraic Variables \\ Common Block /2ZCOMU \\ ABORT $F$ \\ CFRACH (50) 5.5555E+33 \\ CFRHCL (50) 5.5555E+33 CFRHCU (50) $5.5555 E+33$ \\ CFRACF 5.82486000
}

\section{ZDFGAC 0 .}

ZDFMUS 6.8100E-05

ZDNMCP 0.

ZDCCNT(1) $-2.4470 E-08$

ZDCCNT (2) 3.9262E-09

ZDCCNT (3) 9.6127E-08

ZDCCNT (4) 8.6625E-08

ZDCCNT(5)-9.7346E-09

2DCCNT (6) 1.6823E-08

2DCCNT(7)-7.4055E-09

ZDPGAC-0.15578700

DWCILC 0.

DWCOLC 0.

ZDXCON-0.01537870 
CFRHLF 2.52038000 CERHUF 3.03794000 CIRACH(50) $5.5555 E+33$ CIRHCL (50) 5.5555E+33 CIRHCU(50) 5.5555E+33 CIRHLF 1.48056000 CIRHUF 1.82997000

CKACHO 0.63335200 CKBYPO 0.61842000 CKCLRI 0.61279700 CKCORO 0.63335200 CRCTBO 0.60042600 CKESCL 0.60500800 CKESHL 0.60660700 CKHCLO 0.63328400 CKHCUO 0.63524500 CKHLRO 0.63175700 CKHXPO 0.60500800 CKIPCI 0.61281000 CKLHLO 0.63170500 CKMCPO 0.61281300 CKMHLL 0.63170500 CKMHXI 0.63170500 CKMHXO 0.61241500 CKPCHO 0.60500800 CKREFO 0.62689800

CKsHLX 0.62121300 CRTCLO 0.60042600

CKVESI 0.61278300 CKVESO 0.63179500

CPBYPO 4212.49000 CPCLRI 4225.18000

CPCORO 4170.62000 CPCTBO 4249.96000

CPESCL 4241.22000 CPESHL 4238.06000 CPHCLO 4159.44000 CPHCUO 4155.70000 CPHLRO 4176.17000
CKCLRO 0.61278300

CKEHХO 0.61231200

CKGAC 0.60903800

CKHLRI 0.63179500

CKIFDI 0.61278300

CKMCPI 0.61230100

CKMHLO 0.63173500

CKMRPO 0.60500800

CKSCLX 0.60042600

CKTHLO 0.62121300

CPACHO 4170.62000

CPCLRO 4225.21000

CPEHXO 4226.23000

CPGAC 4233.12000

CPHLRI 4176.04000
CPHXPO 4241.22000

CPIPCI 4225.16000 CPLHLO 4176.34000 CPMCPO 4225.15000 CPMHLL 4176.34000 CPMHXI 4176.34000 CPMHXO 4226.01000 CPPCHO 4241.22000 CPREFO 4190.84000 CPSHLX 4205.75000 CPTCLO 4249.96000 CPVESI 4225.21000 CPVESO 4176.04000 CSRACH (50) 5.5555E+33 CSRHCL (50) 5.5555E+33 CSRHCU(50) $5.5555 E+33$ CSRHLF 2.52038000 CSRHUF 3.03794000 DCPCON 15 DCTCON 16

DWACHI 0. DWACHO 0.

DWBYPO 0 . DWCLRI 0 .

DWCORO $O$. DWCTBI 0 .

DWEHXO 0. DWESCL 0 . DWHCLI 0 . DWHCLO 0. DWHCUO 0. DWHLRI 0. DWHXPI 0 . DWHXPO 0. DHIPCI 0. DWLHLO 0. DWMCPO 0. DWMHLA 0 .
CPIFDI 4225.21000

CPMCPI 4226.25000

CPMHLO 4176.24000

CPMRPO 4241.22000

CPSCLX 4249.96000

CPTHLO 4205.75000

CSRACF 5.82486000

DCFCON 14

DHTCDH 12

DWBYPI 0.

DWCLRO 0

DЖСтвO 0

DWESHL 0 .

DWHCUI 0.

DWHLRO 0

DWIFDI 0.

DWMCLI 0.

DWMHLL 0 . 


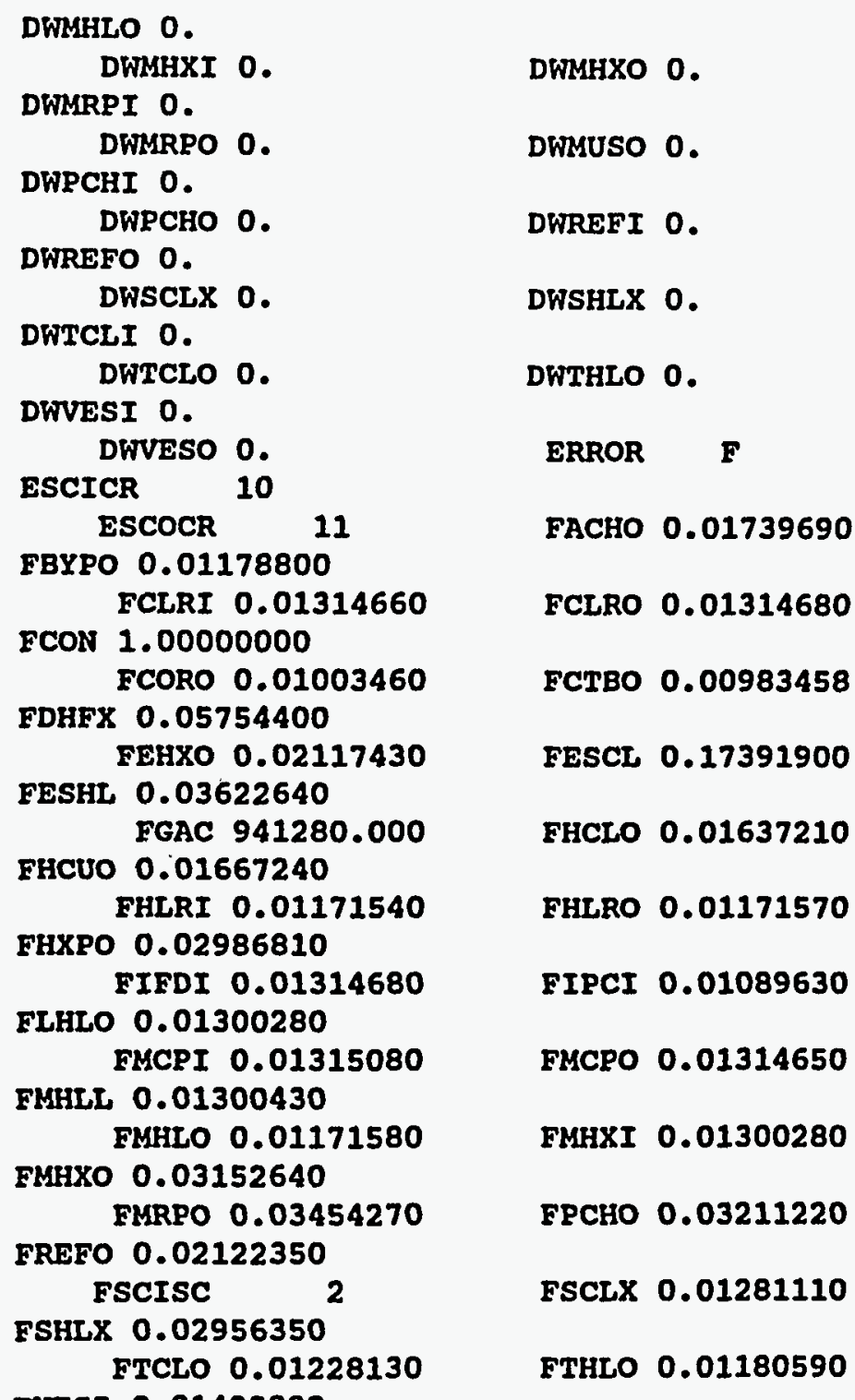

FVESO 0.00825411

HACHI 187933.000 KBYPI 187933.000

HCOLC 334703.000 HCTBI 239306.000

HFFF 175180.000 HGAC 168000.000

HHCUI 187933.000 HHLF 178292.000

HHUF 173687.000 HHXPI 127200.000

HLDSI 333542.000 HMCLI 187951.000

HMHLA 333542.000 HMHLI 333542.000

HMRPI 127200.000 HMUPI 188065.000

HPCHI 127200.000 HREFI 206661.000

HTCLI 127200.000 HVESO 334703.000 HWBYPO 37763.6000 HWCLRI 32469.1000 HWCORO 60310.8000 HWCTBO 76.9571000 HWESCL 5.77484000 HWESHL 674.460000 HWHCLO 180987.000 HWHCUO 175784.000 HWHLRO 40843.6000 HWHXPO 55.5940000 HWIPCI 49886.3000 HWLHLO 42465.8000 HWMCPO 32477.0000 HWMHLL 42179.0000 HWMHXI 42465.8000 HWMHXO 5234.57000
HACF 159027.000

HCILC 187783.000

HESCL 148200.000

HHCLI 187933.000

HHLRI 334703.000

HIFDI 187783.000

HMCPO 187951.000

HMHXI 333542.000

HMUSO 188065.000

HSCLX 127200.000

HWACHO 160305.000

HWCLRO 32462.8000

HWEHXO 9714.27000

HWGAC 8.16541000

HWHLRI 40872.1000

HWIFDI 32462.8000

HWMCPI 32257.0000

HFMHLO 40827.6000

HWMRPO 36.1514000 
НWРСНО 44.5846000

HWREFO 1194.22000

HFSHLX 16484.4000

HWTCLO 6233.71000

HWVESI 90958.5000

HWVESO 34308.2000

IACH

IDVAN

IHCU

IDVANS 1

ISCISC

1

JACH 3.0301E+08

JACHO $3.0301 E+08$

JBYPO 1.1661E+07

JCLRI 0 .

JCORO 0.

JCTBO-3.1387E+08

JESHL 799926.000

JHCL $5.5324 E+08$

JHCU 4.8490E+08 JHCUO 4.8489E+08

JHLRO 0 .

JIFDI 0 .

JHUF 4.8490E+08

JIPCI 0 .

JMCP 6.0000E+06 JMCPI 0 .

JMHXO-3. 1584E+08 JMRPO 1.2377E+06

JPOI $3.1101 \mathrm{E}+08$ JREFC 1.5325E+07

JSHLX $3.1584 E+08$ JTCLO 0.

JTHLO 0. JVESI 0.

JWBYP 0.

JWCLR-172745.000

JWEPP-79515. 3000
HWSCLX 13376.8000

HWTHLO 8153.35000

$\begin{array}{rr}\text { I } & 8 \\ \text { IHCL } & 24\end{array}$

JACF $3.0301 E+08$

JBYP 1.1661E+07

JCLRO 0 .

JEHXO-799926.000

JHCLO $5 \cdot 5330 \mathrm{E}+08$

JHLF $5.5324 E+08$

JHXPO $1.1746 E+06$

JLHLO 0.

JMHLO 0.

JPCHO $1.6182 \mathrm{E}+06$

JREFO $1.5325 \mathrm{E}+07$

JTHDET 3. 1357E+08

JWACC 0 .

JWCPB 0 .
JWESP 0.

JWHLR-1.0650E+06 JWHUC 0 .

JWIFD 0

JWIPR 0.

JWMCL-190740.000 JWMHL -597261.000

JWRRP 0.

JWOPR 0 .

JWPCL-130555.000 JWREF 0 .

JWTCL 0

JWTHL 0 .

КАACHO 0.06663000 KABYPI 0.14800000

KACLRI 0.24966000 KACLRO 0.24966000

RACTBI 1.38000000 KACTBO 100.000000

CAEHXO 1.06000000 RAEMHX 8750.00000

KAESHL 1.00000000 KAGAC 0.24966000 RAHCLO 0.05996700 KAHCUI 0.14800000 KAHLRI 0.24660000 KAHLRO 0.24660000 KAHXPO 10.0000000 KAIFDI 0.24966000 KALHLO 0.24966000 KAMCLA 0.24966000 KAMCPI 0.24966000 KAMCPO 0.24966000 KAMHLO 0.24660000 KAMHXI 0.24966000

KAMRPI 10.0000000 KAMRPO 10.0000000
JFHLC 0.

JWHXP 0.

JWLKL -830245.000

JWMHP-164595.000

JसPCH 0.

JWSHP 0 .

KAACHI 0.14800000

KABYPO 0.03622000

KACORO 0.13326000

KAEEHX 500.000000

KAESCL 100.000000

KAHCLI 0.14800000

KAHCUO 0.05996700

KAHXPI 10.0000000

KAIPCI 0.14800000

KAMCLI 0.24966000

KAMHLL 0.24966000

KAMHXO 2.50000000

KAPCHI 10.0000000 


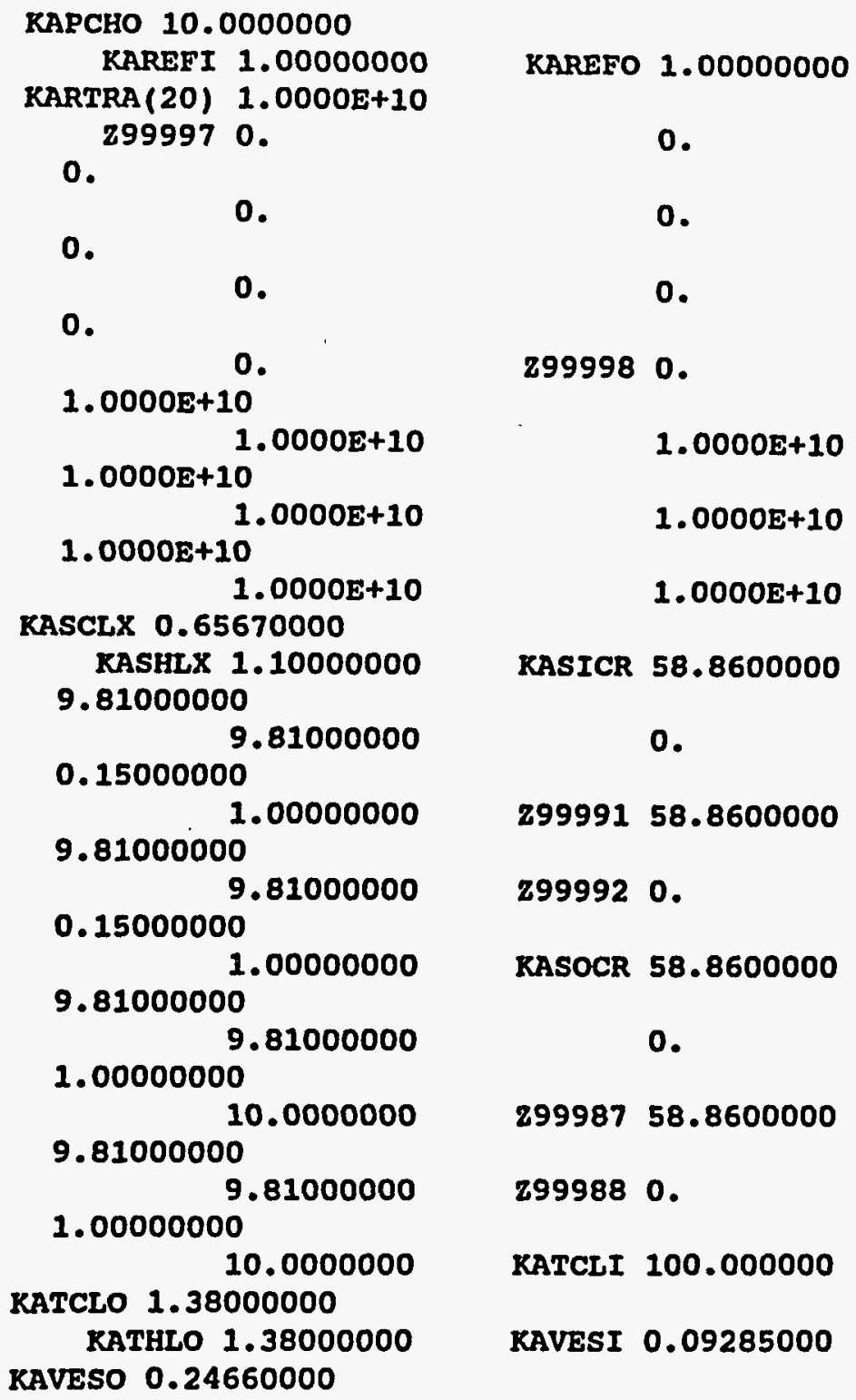

KAREFO 1.00000000
0.
0.
0.
$1.0000 \mathrm{E}+10$
$1.0000 \mathrm{E}+10$
$1.0000 \mathrm{E}+10$

2999980.

KASICR 58.8600000

0 .

29999158.8600000

2999920.

KASOCR 58.8600000

0.

2999880.

KATCLI 100.000000

KAVESI 0.09285000

KBCCOR 4.2748E-04 0.00131134

$2.7879 \mathrm{E}-04$

0.00332481

$1.5695 \mathrm{E}-04$

0.00146622

$7.7441 \mathrm{E}-04$

0.00131134

2.7879E-04

KBDCIL 0 .

KBDCOL 0 .

KBECNT 0.00774000 KBEPOI 0.00800000

KBFCPG 0.02140000 KBWCOR 0.16500000

KCACHO 1.00000000 KCBYPI 0.16000000

RCCANS 0.00100000 KCCLRI 1.00000000 KCCORO 1.00000000 RCCTBI 1.00000000 RCEHXO 1.00000000 KCESCL 1.00000000 KCFACC 0 . KCFACF 0.

RCFCLR 0 . KCFCOR 0 . RCFHCU 0. KCFACH 0.

RCFEPP 0 . KCFESP 0.

KCFHLF 0. KCFHLR 0.

KCFHUF 0 . KCFHXP 0.

KCFIPR 0 . KCFLHL 0 .

\subsection{2}

$7.7441 E-04$

KBCCNT 4.2748E-04

0.00332481

1.5695E-04

KBECOR 0.00774000

KBFCOR 0.02140000

KCACHI 0.16000000

KCBYPO 1.00000000

KCCLRO 1.00000000

ксство 0.

KCESHL 1.00000000

KCFBYP 0 .

KCFHCL 0 .

KCFCTB 0.

KCFHLC 0 .

KCFHUC 0.

KCFIFD 0.

KCFMCL 0 . 
KCFMHL 0. XCFMHP 0.

KCFOPR 0 . KCFPCH 0 .

KCFREF 0 . KCFSHP 0.

KCETHL 0 . KCHCLI 0.16000000 KCHCUI 0.16000000 KCHCUO 1.00000000 KCHLRI 1.00000000 KCHLRO 1.00000000

RCHXPI 0 .

RCHXPO 0 .

KCIPCI 0.16000000 KCLHLO 1.00000000

KCMCPI 1.00000000 KCMCPO 1.00000000

RCMHXI 1.00000000 КСMHXO 1.00000000

RCMRPO 0 . KCPACF 620.000000 КСРСHO 0 . KCPCOR 620.000000 RCPHCU 620.000000 KCPACH 620.000000 KCPHUF 620.000000 RCREFI 0

KCSCLX 1.00000000 KCSHCX 1.00000000

KCTACF 1.00000000 KCTBYP 0.01000000

KCTCLO 1.00000000 KCTCLR 0.01000000

KCTEPP 0.01000000 KCTESP 0.01000000 KCTHLF 1.00000000
KCFMRP 0.

KCFPCL 0.

KCFTCL 0 .

KCHCLO 1.00000000

RCHEHX 1.0000 E-04

КСНМНX $1.0000 \mathrm{E}-04$

KCIFDI 0.30000000

KCMCLI 1.00000000

КСMHLO 1.00000000

RCMRPI 0.

KCPCHI 0 .

KCPHCL 620.000000

KCPHLF 620.000000

KCREFO 0.

KCTACC 0.01000000

KCTCLI 0.

RCTCTB 0.01000000

RCTHLC 0.01000000
KCTHLO 1.00000000 KCTHUC 0.01000000 KCTHUF 1.00000000 RCTIFD 0.01000000 KCTIPR 0.01000000 KCTMCL 0.01000000 KCTMHL 0.01000000

KCTMRP 0.01000000 KCTOPR 0.01000000

KCTPCL 0.01000000 KCTREF 0.01000000

XCTTCL 0.01000000 KCTTHL 0.01000000

KCVCLR 2.51158000 KCVCOR 0.03370000 RCVHCU 0.03370000 KCVBYP 0.03370000 XCVCTB 4500.00000 KCVEPP 12.0000000 RCVESO 1.00000000 KCVESP 12.0000000 RCVHLR 3.75818000 KCVHUC 0.03370000

KCVIFD 0.37140000 KCVIPR 0.48000000

RCVMCL 4.70859000 KCVMHL 3.76312000

KCVMRP 500.000000 KCVOPR 0.48000000

RCVPCL 3.46029000 KCVREF 15.0000000 KCVTCL 24.0000000 KCVTHL 24.0000000 KDBCOR 0.26360000 KDBCPG 0.26360000 KDCCC 0.00228600 KDCLRI 0.32550000
KCTHLR 0.01000000

KCTHXP 0.01000000

KCTLHL 0.01000000

ХСTMHP 0.01000000

КСTPCH 0.01000000

RCTSHP 0.01000000

RCVACC 0.03370000

KCVHCL 0.03370000

RCVACH 0.03370000

RCVESI 0.23000000

RCVHLC 0.03370000

KCVHXP 1200.00000

RCVLHL 3.04336000

KCVMHP 29.1000000

KCVPCH 300.000000

KCVSHP 29.1000000

KDACHO 0.00254000

KDBYPO 0.09500000

KDCLRO 0.32550000 


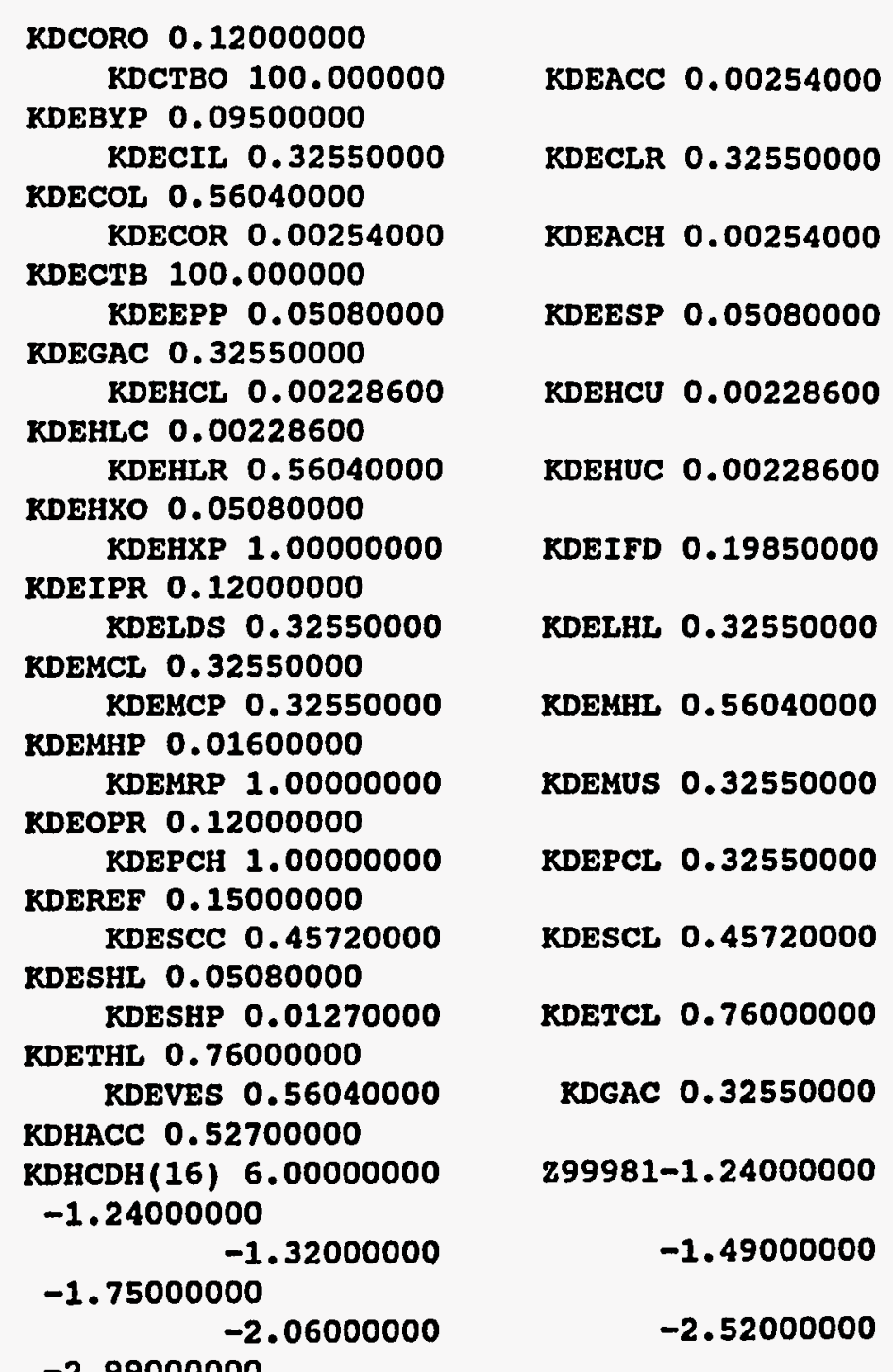

$-2.99000000$ 299982-10.0000000 1.00000000

$$
\begin{gathered}
2.00000000 \\
5.00000000000
\end{gathered}
$$

KDHCLO 0.00228600 KDHCLR-13.5700000 KDHCUO 0.00228600 KDHEHX 2792.00000

KDHESP 3.00000000 KDHGAC $5.0000000 \mathrm{C}$

KDHHLR 12.5000000 KDHHUC 0.52700000

KDHIFD 0.

KDHIPR 3.00000000

KDHLRI 0.56040000 KDHLRO 0.56040000

KDHMHL 0. KDHMHP 0.

KDHMRP 0 . KDHOPR 4.25300000

KDHPCL-4.88000000 KDHREF 0 .

KDHTCL-3.00000000 KDHTHL 3.00000000 KDIFDI 0.32550000 KDIPCI 0.12000000 KDMCLA 0.32550000 KDMCPI 0.32550000 XDMHLL 0.32550000 KDMHLO 0.56040000 KDMHXO 0.01600000 KDMRPO 1.00000000 KDRCOR 0.08640000 KDRCPG 0.08640000 KDSCLX 0.45720000 KDSHLX 0.01270000

o.
3.00000000

6.00000000

кDHCTB 0 .

KDHEPP 0.

KDHKLC 0.52700000

KDHHXP 0.

KDHLHL-1.83000000

KDHMCL 0.

KDHMHX 2391.00000

RDHPCH 0 .

KDHSHP 0.

KDHXPO 1.00000000

KDLHLO 0.32550000

KDMCPO 0.32550000

KDMHXI 0.32550000

KDPCHO 1.00000000

KDREFO 0.15000000

KDTCLO 0.76000000

KDVESO 0.56040000 
KEACHO 0.

KEBYPO 0.

KECLRO 4.5700E-05 KECORO 0 .

KEEHXO 4.5700E-05 KEESCL 4.5700E-05

KEFPOI 3.2000E-11 REGAC 4.5700E-05

KEHCUO 0.

KEHLRI 4.5700E-05

REHXPO 4.5700E-05 KEIFDI 4.5700E-05

RELHLO 4.5700E-05 KEMCPI 4.5700E-05

KEMHLL 4.5700E-05 KEMHLO 4.5700E-05

KEMHXO 4.5700E-05 KEMRPO 4.5700E-05

RERACC 0.

KERBYP 0.

KERCLR 4.5700E-05 KERCOL 4.5700E-05

KERHCL 0.

KERHCU 0.

KERCTB 4.5700E-05

KEREFO 4.5700E-05

KERESP 4.5700E-05

KERGAC 4.5700E-05

KERHLR 4.5700E-05

KERHUC 0 .

KERIFD 4.5700E-05

KERIPR 0.

KERLHL 4.5700E-05

KERMCL 4.5700E-05

KERMHL 4.5700E-05

KERMHP 4.5700E-05

KERMUS 4.5700E-05
KECLRI 4.5700E-05

KECTBO 4.5700E-05

KEESHL 4.5700E-05

KEHCLO 0.

KEHLRO 4.5700E-05

KEIPCI 0 .

KEMCPO 4.5700E-05

KEMHXI 4.5700E-05

REPCHO 4.5700E-05

RERCIL 4.5700E-05

KERCOR 0.

KERACH 0.

KEREPP 4.5700E-05

KERHLC 0.

KERHXP 4.5700E-05

KERLDS 4.5700E-05

KERMCP 4.5700E-05

KERMRP 4.5700E-05
KEROPR 0.

KERPCL 4.5700E-05 KERREF 4.5700E-05;

KERSHP 4.5700E-05 KERTCL 4.5700E-05 KERVES 1.5200E-06 KESCLX 4.5700E-05 KETCLO 4.5700E-05 KETHLO 4.5700E-05 KEVESO 1.5200E-06 KEY

0

KFABYP 0.03622000 KFACIL 0.24966000

RFACOL 0.24660000 KFACOR 0.06663000

KFACTB 100.000000 KFAEPP 1.06000000

KFAGAC 0.24966000 KFAHCL 0.05996700

KFAHLC 0.05996700 KFAHLR 0.24660000 KFAKXP 10.0000000 KFAIFD 0.09285000 KFALDS 0.24966000 KFALHL 0.24966000 KFAMCP 0.24966000 KFAMHL 0.24660000 KFAMRP 10.0000000 KFAMUS 0.24966000 KFAPCH 10.0000000 KFAPCL 0.24966000 KFASCC 0.65670000 KFASHP 1.10000000 KFATHL 1.38000000 KFAVES 0.24660000 KFDACF 3870.00000 KFDCOR 3870.00000
KERPCH 4.5700E-05

KERSCC 4.5700E-05

KERTHL 4.5700E-05

KESHLX 4.5700E-05

KEVESI 4.5700E-05

KFAACC 0.06663000

KFACLR 0.24966000

KFAACH 0.06663000

RFAESP 1.00000000

KFAHCU 0.05996700

KFAHUC 0.05996700

KFAIPR 0.14800000

KFAMCL 0.24966000

KFAMHP 2.50000000

KFAOPR 0.13326000

KFAREF 1.00000000

KFATCL 1.38000000

KFCCON 0.

KFDHCL 3870.00000 
KFDHCU 3870.00000 KFDACH 3870.00000

KFDHUF 3870.00000 KFEDET 1.00000000

KFHCOR 0.52700000 KDHHCL 0.52700000

KDHBYP 0.52700000 KDHACH 0.52700000

KFLACC 0.52700000 KFLBYP 0.99800000

KFLCOR $\mathbf{0 . 5 2 7 0 0 0 0 0}$ KFLHCL 0.52700000

KFLACH 0.52700000 KFLCTB 0 .

RFLESP 6.00000000 KFLGAC 5.00000000 RFLHLR 15.2400000 KFLHUC 0.52700000

KFLIFD 4.00000000 KFLIPR 3.00000000 RFLLHL 12.1900000 RFLMCL 18.8600000 KFLMHL 15.2600000 KFLMHP 40.0000000 KFLMUS 0 .

KFLOPR 3.00000000

KFLPCL 13.8600000 KFLREF 0 .

KFLTCL 20.0000000 KFLTHL 20.0000000 KFRCOR 0.04400000 KFRCPG 0.04400000 KFTACF 0.00127000 KFTCOR 0.00127000 KFTHCU 0.00127000 KFTACH 0.00127000 KFTHUF 0.00127000
KFDHLF 3870.00000

KFFISC 1.15000000

KDHHCU 0.52700000

RFICON 1.05000000

KFLCLR 10.0600000

RFLHCU 0.52700000

RFLEPP 24.0000000

KFLHLC 0.52700000

KFLHXP 0 .

KFLLDS 0.

KFLMCP 0.

KFLMRP 0.

KFLPCH 0.

KFLSHP 12.2000000

KFPPOI $1.5000 E+10$

KFSCON 1.00000000

KFTHCL 0.00127000

KFTHLF 0.00127000
KFVACF 0.03370000

KFVHCL 0.03370000

KFVHCU 0.03370000

KFVHLF 0.03370000

KFVHUF 0.03370000 KGBCI

KGBCOL

$F$

KGFGAC 0.07400000

KGIPOI 0.05600000

KGTCOR 5.0000E-0

KGTCNT 5.0000E-04

KHOGAC 168000.000

KHACOR 0.90000000

KHMEHX 188065.000

KHMMUS 188065.000

KHPCCS 148200.000

KHSACF 1.00000000

KHSSCC 127200.000

KHSHLF 1.00000000

KHUCOR 1.07400000 KHWACC 0 .

KHWCLR 600.000000 KHWCTB 0.

KHWESP 0 KHWHLC 0.

KHWHUC 0. KHWHXP 0.

KHWIPR 0.

KHWLHL 500.000000

KHWMHL 500.000000 KHWMHP 500.000000 KHWOPR 0. KHWPCH 0 .

KHWREF 0. KHWSHP 0.

KHWTHL 0. KIIPOI 1.00000000
KFVCOR 0.03370000

KFVACH 0.03370000

KFWISC 5.3638E-04

KGCGAC 1.00000000

KGPPOI 0.01130000

KGXPOI 0.00300000

KHCCCS 190857.000

KHMMHX 188466.000

KHSCCS 127200.000

KHSHUF 1.00000000

KHWBYP 0 .

KHWEPP 350.000000

KHWHLR 900.000000

KHWIFD 0.

KHWMCL 350.000000

KHWMRP 0.

KHWPCL 350.000000

KHWTCL 0.

KIPPOI 1.00000000 
KISPOI 1.00000000 KIXPOI 1.00000000

KJOCOR 3.3000E+08 KJOCPG $3.3000 \mathrm{E}+08$

KJNCNT 3.1101E+08 KJNCOR 3.3000E+08

KJPMCP (22) 2.00000000

299953(11) 0 .

KKAACF 66.0000000 KKACOR 66.0000000

KKAHCU 66.0000000 KKAACH 66.0000000

KKAHUE 66.0000000 KRCACC 1.00000000

KKCCIL 0.30000000 KRCCLR 1.00000000

KKCCOR 1.00000000 KKCHCL 1.00000000

KKCACH 1.00000000 RKCCTB 0.

KRCESP 1.00000000 KKCGAC 1.00000000

KKCHLR 1.00000000 KKCHUC 1.00000000

KKCIFD 0.23000000 KKCIPR 0.16000000

KKCLHL 1.00000000 KKCMCL 1.00000000 KKCMCS 1.00000000 KKCMHL 1.00000000 KKCMRP 0 .

KKCMUS 1.00000000 KKCPCH 0.

KKCPCL 1.00000000

KKCSCC 1.00000000 KKCSHP 1.00000000

KKСTHL 1.00000000
KJOCNT 3.1101E+08

KJOMCP 6.0000E+06

KJNCPG 3.3000E+08

z99954(11) 2.00000000

KKAHCL 66.0000000

KKAHLF 66.0000000

KRCBYP 1.00000000

KKCCOL 1.00000000

RKCHCU 1.00000000

KKCEPP 1.00000000

KRCHLC 1.00000000

RRCHXP 0.

KRCLDS 1.00000000

RKCMCP 1.00000000

KKCMHP 1.00000000

KKCOPR 1.00000000

KKCREF 0 .

KRCTCL 1.00000000
KKFACF 154.600000

RKFCOR 154.600000

KKFHCL 154.600000

KRFACH 154.600000

KKFGAC 0.01000000

KKFHUF 154.600000

KKFMUS 0.01000000

KKIMCP 1000.00000

KKIMUS 1.00000000

KKLCON 5.0000E-06

KKOACF 2.25000000

KROHCL 2.25000000

KROHCU 2.25000000

KKOHLF 2.25000000

KKOHUF 2.25000000

KKPCOL 1000.00000

KRTCON 1.00000000

RKWCOL 1000.00000

KLACHO 0.52700000

RLACOL 10.0000000

KLAGAC 10.0000000

KLASCC 50.0000000

KLBYPO 0.99800000

KLCCOR 0.01430000

0.29600000

0.03050000

1.1000E-04

KLCCNT 0.01430000

0.11100000

0.29600000

3.00000000

1.1000E-04

KLCLRO 10.0600000

KLCORO 3.00000000

KLEHXO 24.0000000

KLESHL 6.00000000
KKFCON 1.00000000

KKFHCU 154.600000

KKFHLF 154.600000

KKIGAC 1000.00000

KRISCC 1.00000000

KKOCOR 2.25000000

KKOACH 2.25000000

KKPCIL 1000.00000

KRWCIL 1000.00000

KLACIL 10.0000000

KLAMCP 5.00000000

KLCCC 0.51700000

0.11100000

3.00000000

0.03050000

1.13000000

KLCLRI 18.8600000

KLCTBO 0.

KLHCLO 0.52700000 


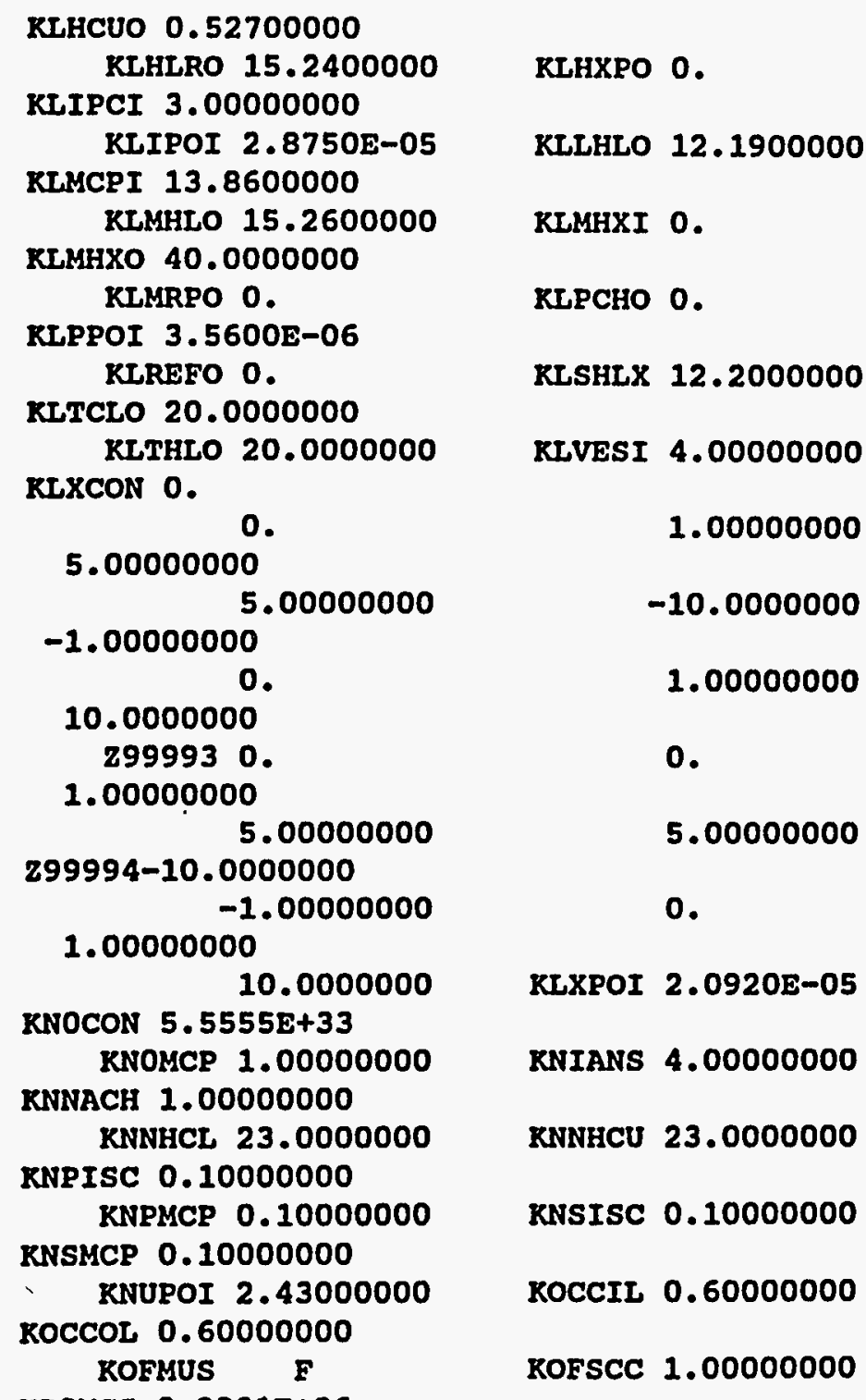

KPBCCS 150000.000 KPCCCS 3.1180E+06

KPCCIL 350000.000 KPECCS 0.01000000

KPFISC 0.80000000

KPLCON 150000.000

KPLCOR 1.70000000 KPMCIL 1000.00000

KPMCOL 1000.00000 KPRMCP (18) 2.00000000 2999510.

3300.00000

13000.0000
110000.000
200000.000
310000.000
$299952-1.00000000$
0.10000000

0.60000000

0.20000000

0.80000000

2.00000000

KPSACH(50) 1.00000000

KPSHCL (50) 1.00000000 KPSHCU (50) 1.00000000 KPTMCP 1.50000000

KPUCOR 1.49000000 RPWMCP (22) 2.40000000 299955 (11) 0 .

299956 (11) 2.40000000

KRACOR 7.40000000

KRBCOR 9.10000000

KRRREF 29.0000000

KRSISC 0.20000000

KSAACF 2.5400E-04

KSABYP 0 .

KSACOR 2.5400E-04

KSAHCL $2.5400 \mathrm{E}-04$
KPBSCC 150000.000

KPCCOL 290000.000

KPLCCS 150000.000

KPLMUS 100000.000

KPMMCS 21000.0000

o.

50000.0000

310000.000

0 .

0.40000000

$\stackrel{1}{\Delta}$

1.00000000

KPSCON 1.7011E+06

KPSISC $1.3584 \mathrm{E}+06$

KROCNT 0 .

KRFCOR-0.00125000

KSAACC 0.

KSACLR 30.8643000

KSAHCU 2.5400E-04 
KSAACH 2.5400E-04 KSACTB 0 .

RSAESP 0 .

KSAHLC 0.

RSAHLR 26.8250000

KSAHUC 0 .

KSAHXP 0.

KSAIFD 7.48413000

KSALHL 37.3991000

KSAMCL 57.8628000

RSAMHP 40.0000000 KSAMRP 0.

KSAPCH 0 .

KSAPCL 42.5227000

KSASHP 0 .

KSATCL 0 .

KSFACF $7.6240 \mathrm{E}-04$ KSFCOR 7.6240E-04

KSFHCU 7.6240E-04 KSFACH 7.6240E-04

KSFHUE 7.6240E-04 KSOACF 2.500OE-05

KSOACH 2.500OE-05 KSOHCL 1.5000E-05

KSOHLF 1.5000E-05 KSOHUF 1.500OE-05

KSSPOI 4.0800E-24 KSXPOI $2.7200 \mathrm{E}-22$

KTDDET 0.25000000 KTDISC 0.03000000

KTFDET 0.02500000 KTLCIL 0.25000000

RTLCON 1.00000000 KTLMUS 5.00000000

KTPDET 0.03000000 KTSCON 45.0000000 KTSISC 55.0000000
KSAEPP 26.5000000

KSAHLF $2.5400 E-04$

KSAHUF $2.5400 E-04$

RSAIPR 0

RSAMHI 26.8602000

KSAOPR 0 .

KSAREF 0.

KSATHL 0.

KSFHCL $7.6240 \mathrm{E}-04$

RSFHLF $7.6240 \mathrm{E}-04$

KSOCOR 2.5000E-05

KSOHCU 1.5000E-05

KSSISC-1.0000E+10

KTCMCP 2.00000000

KTDOSC 0.12000000

KTLCOT 0.25000000

KTNCON 600.000000

KTSEHX 0.
KTSMHX 0

RTWDET 0.25000000

KUCACH 1.30500000

KUCHCL 1.21508000

KUCHCU 1.21508000

KUFHCL 0.03795000

KUFHCU 0.03795000

KUGACH 1.55400000 KUGCOR 1.55400000

KUGHCU 1.44693000 KUWEHX 8.7000E-05

KVAGAC 21.0000000 KVFICR 0.03000000

KVSICR 0.01500000 KVSOCR 1.00OOE+10 KWOMCP 2144.00000 KWCCOR 2144.00000 KWECCS 104.000000 KWFISC 0.08000000 KWLMUS 14.6000000 KWLLDS 14.6000000 KWNCON 0.

KWRICR(28) $1.00000000 \mathrm{z} 99989(14)-15.2100000$ z99990 (14) 1.00000000 KWROCR(20) 1.00000000 0 .

$$
\begin{gathered}
-0.25000000 \\
-3.11000000 \\
-4.00000000 \\
-6.58000000 \\
-8.53000000 \\
299986-1.00000000 \\
-0.80000000 \\
-0.50000000 \\
-0.40000000 \\
-0.25000000 \\
-0.12500000
\end{gathered}
$$

KWCCON 2144.00000
KTTDET 2.00000000

KUCCOR 1.30500000

KUFCOR 0.03795000

KUFACH 0.03795000

KUGHCL 1.44693000

KUWMHX $2.6000 E-04$

KVFOCR 0 .

KWCCCS 2144.00000

KWLCCS 14.6000000

RWLCON 14.6000000

2999850.

$-2.02000000$

$-4.60000000$

$-8.53000000$

$-0.60000000$

$-0.30000000$

0. 
1.00000000

KWSCCS 2800.00000

KWXCON 1.20000000

KXOICR-0.15000000

KXMICR 0.60000000 KXMOCR 0 .

KXNOCR-0.80000000 KXSICR 0.01700000

KZOGAC 5.00000000 LCNT-2.7054E-07

MCRACH 3.81319000 MCRCOR 1.99977000

MCRHCU 2.25843000 MIRACH 2.46468000 MIRHCL 1.33148000 MIRHCU 1.46455000 MMUSO 0.

MPRACH 4.85824000

MPRHCL 1.50195000 MPRHCU 1.73936000

MSRACH 6.27408000 MSRCOR 3.17389000

MSRHCU 3.48308000 MUACHO 3.94985-04

MUCLRI 7.2425E-04 MUCLRO 7.2451E-04

MUCTBO 9.8605E-04 MUEHXO 7.3303E-04

MUESHL 8.4429E-04 MUGAC 7.9493E-04

MUHCUO 2.7469E-04 MUHLRI 4.2217E-04 MUHXPO $8.7855 \mathrm{E}-04$ MUIFDI 7.24515-04 MULHLO 4.2366E-04 MUMCPI 7.3323E-04 MUMHLL $4.2366 \mathrm{~g}-04$
KWSISC 171.520000

KX0OCR-0.80000000

KXNICR-0.60000000

KXSOCR 1.0000E+10

LGAC 4.63000000

MCRHCL 1.99977000

MIRCOR 1.33148000

MIDSI 0.

MPRCOR 1.50195000

MPRMCP 7.26493000

MSRHCL 3.17389000

MUBYPO $6.2862 \mathrm{E}-04$

MUCORO 3.9498E-04

MUESCL $8.7855 \mathrm{E}-04$

MUнСLо 2.5096E-04

MUHLRO 4.2280E-04

MUIPCI $7.2402 E-04$

MUMCPO $7.2396 \mathrm{E}-04$
MUMHLO 4.2316E-04

MUMHXO 7.3117E-04

MUMRPO 8.7855E-04

MUREFO 4.9822E-04

MUSCLX 9.8605E-04

MUTCLO $9.8605 \mathrm{E}-04$ MUTHLO 5.8445E-04

MUVESO 4.2217E-04 MXNERR 100000

NERROR

NHLF 2.00000000 ।

NLL $2.2421 \mathrm{E}-44$

NMCP 1.00000000

NSPCON 1.00000000 ODCCON

OFICON

OEIICR F

OFRCOR OMSKER

oscrsc

OSCMCP $5.5555 \mathrm{E}+33$

OSEISC

OSEOSC

$\mathbf{T}$

OSFOSC F OSIISC $\quad F$

PACHI 3.1991E+06 PACHO 1.7083E+06

PBYPO $3.1469 E+06$ PCCC 1.7308E+06

PCLRI 3.2675E+06 PCLRO 3.4001E+06

PCORO 1.8104E+06 PCTBI 150000.000

PEHXO 1.6502E+06 PESCL 150000.000

PGAC 1.5864E+06 PHCLI 3.1991E+06
MUMHXI 4.2366E-04

MUPCHO 8.7855E-04

MUSHLX 5.8445E-04

MUVESI $7.2451 \mathrm{E}-04$

NACF 3.00000000

NHUF 3.00000000

NPHMCP 7.26493000

OFFGAC F

OFIOCR F

OPRINT T

OSCPPS

OSFISC F

OsIosc F

PBYPI 3.1991E+06

PCILC 350000.000

PCOLC 290000.000

РСTBO 150051.000

PESHL 117542.000

PHCLO $1.7077 E+06$ 
PHCUI 3.1991E+06 PHCUO $1.6608 \mathrm{E}+06$

PHLRO 1.6983E+06 PHXPI 150000.000

PIFDI 3.4001E+06 PIPCI 3.1991E+06

PLHLO 1.6906E+06 PMCLI 3.2931E+06

PMCPO 3.2931E+06 PMHLA $1.6906 \mathrm{E}+06$

PMHLO 1.6871E+06 PMHXI 1.6906E+06

PMRPI 150000.000 PMRPO 150000.000

PMUSO 1.6906E+06 PPCHI 150000.000

PRACHO 2.60095000 PRBYPO 4.28197000 PRCLRO 4.99561000 PRCORO 2.60095000

PREFI 1.0000E+06 PREFO 1000000.00 PRESCL 6.15881000 PRESHL 5.89861000

PRHCLO 1.64830000 PRHCUO 1.79702000

PRHLRO 2.79490000 PRHXPO 6.15881000

PRIPCI 4.99193000 PRLHLO 2.80089000

PRMCPI 5.06093000 PRMCPO 4.99149000

PRMHLO 2.79741000 PRMHXI 2.80089000

PRMRPO 6.15881000 PRPCHO 6.15881000

PRSCLX 6.97953000
PHLRI $1.8416 \mathrm{E}+06$

PHXPO 150000.000

PLDSI $1.6906 \mathrm{E}+06$

PMCPI $1.6516 \mathrm{E}+06$

PMHLL 1.6906E+06

PMHXO 1.6704E+06

PMUPI 100000.000

PPCHO 150000.000

PRCLRI 4.99365000

PRCTBO 6.97953000

PREHXO 5.05941000

PRGAC 5.52519000

PRHLRI 2.79044000

PRIFDI 4.99561000

PRMCP 227334.000

PRMHLL 2.80089000

PRMHXO 5.04548000

PRREFO 3.33062000
PRSHLX 3.95688000 PRTHLO 3.95688000 PRVESI 4.99561000 PSACHO 55645.0000

PSBYPO 12579.6000

PSCLRI 8332.30000 PSCLRO 8323.77000 PSCORO 55645.0000 PSCTBO 3682.75000 PSESCL 4930.89000 PSESHL 5473.49000 PSHCLO 270704.000 PSHCUO 196327.000 PSHLRO 44273.9000 PSHLX 181766.000 PSIFDI 8323.77000 PSIPCI 8339.75000 PSMCPI 8047.46000 PSMCPO 8341.69000

PSMHLO 44149.3000 PSMHXI 43976.6000

PSMRPO 4930.89000 PSPCHO 4930.89000 PSSCLX 3682.75000 PSSHLX 15679.3000 PSTHLO 15679.3000 PSVESI 8323.77000 PTCLI 232037.000 PTCLO 262089.000 PTMCP 13 PVESI $3.1180 \mathrm{E}+06$ PWACF 201030.000 PWFFF 689249.000 PWHUF 847871.000 QACF 5.7094E+06 QCFFF 2.6034E+07 QCHLF 2.6277E+07
PRTCLO 6.97953000

PRVESO 2.79044000

PSCISC 6

PSCLX 262089.000

PSEHXO 8053.77000

PSGAC 6434.33000

PSHLRI 44496.8000

PSHXPO 4930.89000

PSLHLO 43976.6000

PSMHLL 43976.6000

PSMHXO 8111.53000

PSREFO 25867.8000

PSTCLO 3682.75000

PSVESO $\mathbf{4 4 4 9 6 . 8 0 0 0}$

PTHLO 149999.000

PVESO $1.8416 \mathrm{E}+06$

PWHLF $1.2327 E+06$

QCACE $3.3257 E+07$

QCHUF $2.7756 E+07$ 


\begin{tabular}{|c|c|c|}
\hline \multicolumn{3}{|l|}{ QFFF $5.9333 E+06$} \\
\hline QHACF 8.8725E+06 & QHHLF & $2.0456 \mathrm{E}+07$ \\
\hline QHHUF $1.5554 \mathrm{E}+07$ & & \\
\hline QHLF 1.0426E+07 & QHUF & $9.1366 \mathrm{E}+06$ \\
\hline QIACF $2.1370 E+07$ & & \\
\hline QIFFF $1.5274 E+07$ & QIHLF & $1.5436 \mathrm{E}+07$ \\
\hline QIHUF $1.6720 \mathrm{E}+07$ & & \\
\hline QSACF $4.6494 \mathrm{E}+07$ & QSFFF & $2.8895 \mathrm{E}+07$ \\
\hline QSHLF $2.8642 \mathrm{E}+07$ & & \\
\hline QSHUF 3.1948E+07 & RACH & 0. \\
\hline RACHI 1098.47000 & & \\
\hline RACHO 1074.10000 & RAREA & 0 . \\
\hline RBYPI 1098.47000 & & 1094.48000 \\
\hline RCLRI 1098.48000 & & \\
\hline $\begin{array}{l}\text { RCLRO } 1098.49000 \\
\text { RCORO } 1074.10000\end{array}$ & RCOR & 0. \\
\hline $\begin{array}{l}\text { RCROD 0. } \\
\text { RCTBO } 1104.88000\end{array}$ & RCTBI & 1092.09000 \\
\hline REACHO 172775.000 & REBYPO & $1.4760 \mathrm{E}+06$ \\
\hline RECLRI 3.8594E+06 & & \\
\hline $\begin{array}{l}\text { RECLRO 3.8580E+06 } \\
\text { RECTBO } 2.8394 \mathrm{E}+06\end{array}$ & RECORO & $4.0813 E+06$ \\
\hline $\begin{array}{l}\text { REEHXO } 140173.000 \\
\text { REESHL } 6257.58000\end{array}$ & REESCL & 541.218000 \\
\hline $\begin{array}{l}\text { REGAC } 1.0000 \mathrm{E}-04 \\
\text { REHCUO } 214141.000\end{array}$ & REHCLO & 235035.000 \\
\hline $\begin{array}{l}\text { REHLRI 1.1541E+07 } \\
\text { REHXO } 1098.79000\end{array}$ & REHLRO & $1.1523 \mathrm{E}+07$ \\
\hline REHXPO 11522.2000 & REIFDI & $3.8580 \mathrm{E}+06$ \\
\hline REIPCI $2.4009 E+06$ & & \\
\hline $\begin{array}{l}\text { RELHLO 6.5977E+06 } \\
\text { REMCPO } 3.8611 E+06\end{array}$ & REMCPI & $3.8123 \mathrm{E}+06$ \\
\hline REMHLL $6.5528 E+06$ & REMHLO & $1.1513 E+07$ \\
\hline $\begin{array}{l}\text { REMHXI 6.5977E+06 } \\
\text { REMHXO } 18766.4000\end{array}$ & REMRPO & 6691.42000 \\
\hline
\end{tabular}

REREFO 60214.4000 RESCLX 1.9768E+06 RESHL 1102.01000 RESPOI -0.58127600 RETCLO 1.5637E+06 REVESI 6.3262E+06 REVESO 1.1541E+07 RGAC 1100.71000 RHACHO-1.7118E-04 RHCLI 1098.47000 RHCLO 1036.40000 RHCLRO-9.6798E-05 RHCORO-1.7118E-04

RHCUI 187933.000 RHCUO 1045.93000 RHESCL-6.3995E-05 RHESHL - 7 . 1650E-05 RHHCLO-2 2835E-04 RHHCUO-2 . 1608E-04 RHHLRO-1.6326E-04 RHHXPO-6.3995E-05

RHIPCI $-9.6898 \mathrm{E}-05$ RHLHLO-1.6303E-04 RHLRO 1077.97000 RHMCPI-9.5023E-05 RHMHLL-1.6303E-04 RHMHLO-1.6316E-04 RHMHXO-9.5443E-05 RHMRPO-6.3995E-05 RHREFO- 1.4430 E-04 RHSCLX-3.7841E-05 RHTCLO-3.7841E-05 RHTHLO-1.2535E-04 RHVESO $-1.6344 \mathrm{E}-04$ RHXPI 1100.00000 RICR 0 .

RIFDI 1098.49000
RESCL 1102.80000

RESHLX 55311.9000

RETHLO 2.6384E+06

REXPOI-2.98575000

RHBYPO-1.1623E-04

RHCLRI-9.6851E-05

RHCTBO-3 . 7841E-05

RHEHXO-9.5065E-05

RHGAC-8.2256E-05

RHHLRI-1.6344E-04

RHIFDI-9.6798E-05

RHLRI 1077.89000

RHMCPO-9.6910E-05

RHMHXI-1.6303E-04

RHPCHO-6. 3995E-05

RHSHLX-1.2535E-04

RHVESI-9.6798E-05

RHXPO 1102.80000

RIPCI 1098.47000 
RLHLO 1078.08000 RMCLI 1098.47000

RMCPO 1098.47000 RMHLL 1078.08000

RMHXI 1078.08000 RMHXO 1098.72000

RMRPO 1102.80000 ROCR 0 .

RPCHO 1102.80000 RREFC 0 .

RREFO 1085.92000 RSCISC 3

RSHLX 1092.09000 RSPOI- 0.58127600

RTCLO 1104.88000 RTHLO 1092.09000

RVESO 1077.89000 RXESM-3.56703000 SSCISC TOANS 0 .

TACHO 85.6446000 TBYPO 52.4385000

TCCC 131.141000 TCFFF 234.715000

TCHUF 280.782000 TCLRI 44.4626000

TCORO 85.6446000 TCSDET 29.9297000

TDAY 0 .

TDHCDH $1.0000 \mathrm{E}+10$

TESCL 34.9428000 TESHL 36.7836000

TGAC 39.6870000 TGGAC 39.6870000 THCHLF 426.651000 THCHUF 365.747000

THCLO 131.141000
RMCPI 1098.79000

RMHLO 1078.02000

RMRPI 1100.00000

RPCHI 1100.00000

RREFI 1100.00000

RSCLX 1104.88000

RTCLI 1104.88000

RVESI 1098.49000

RXPOI-2.98575000

TACHI 44.4794000

TCACF 214.000000

TCHLF 312.079000

TCLRO $\mathbf{4 4 . 4 4 3 4 0 0 0}$

TCTBO 29.9297000

TEHXO 43.8247000

TFFF 225.170000

THCACF 284.127000

THCLI $\mathbf{4 4 . 4 7 9 4 0 0 0}$
THCUI 44.4794000

THLRI 80.1483000

THLRO 80.0271000

THSDET 56.8998000

THWACF 140.472000

THWHUF 187.396000 THXPO 34.9428000

TIPCI 44.4794000 TLHLO 79.8647000

TMCPO 44.4838000 TMHLL 79.8647000

TMHXI 79.8647000 TMHXO 43.9586000

TMRPO 34.9428000 TPCHO 34.9428000

TSACHO 204.636000 TSBYPO 236.395000

TSCLRI 238.518000 TSCLRO 240.786000 TSCORO 207.452000 TSCTBO 112.703000 TSESCL 112.693000 TSESHL 105.558000 TSHCLO 204.618000 TSHCUO 203.282000 TSHLRO 204.352000 TSHLX 56.8998000 TSIFDI 240.786000 TSIPCI 237.321000 TSMCPI 203.015000 TSMCPO 238.960000 TSMHLO 204.035000 TSMHXI 204.136000 TSMRPO 112.693000 TSPCHO 112.693000 TSSCLX 130.201000 TSSHLX 118.528000
THCUO 120.837000

THOUR 0.

THWHLF 200.415000

TIFDI $\mathbf{4 4 . 4 4 3 4 0 0 0}$

TMCPI 43.8101000

TMHLO 79.9592000

TMIN 0.

TREFO 67.5966000

TSCISC 5

TSCLX 29.9297000

TSEHXO 202.974000

TSGAC 201.100000

TSHLRI 208.289000

TSHXPO 112.693000

TSLHLO 204.136000

TSMHLL 204.136000

TSMHXO 203.558000

TSREFO 180.355000

TSTCLO 130.201000 


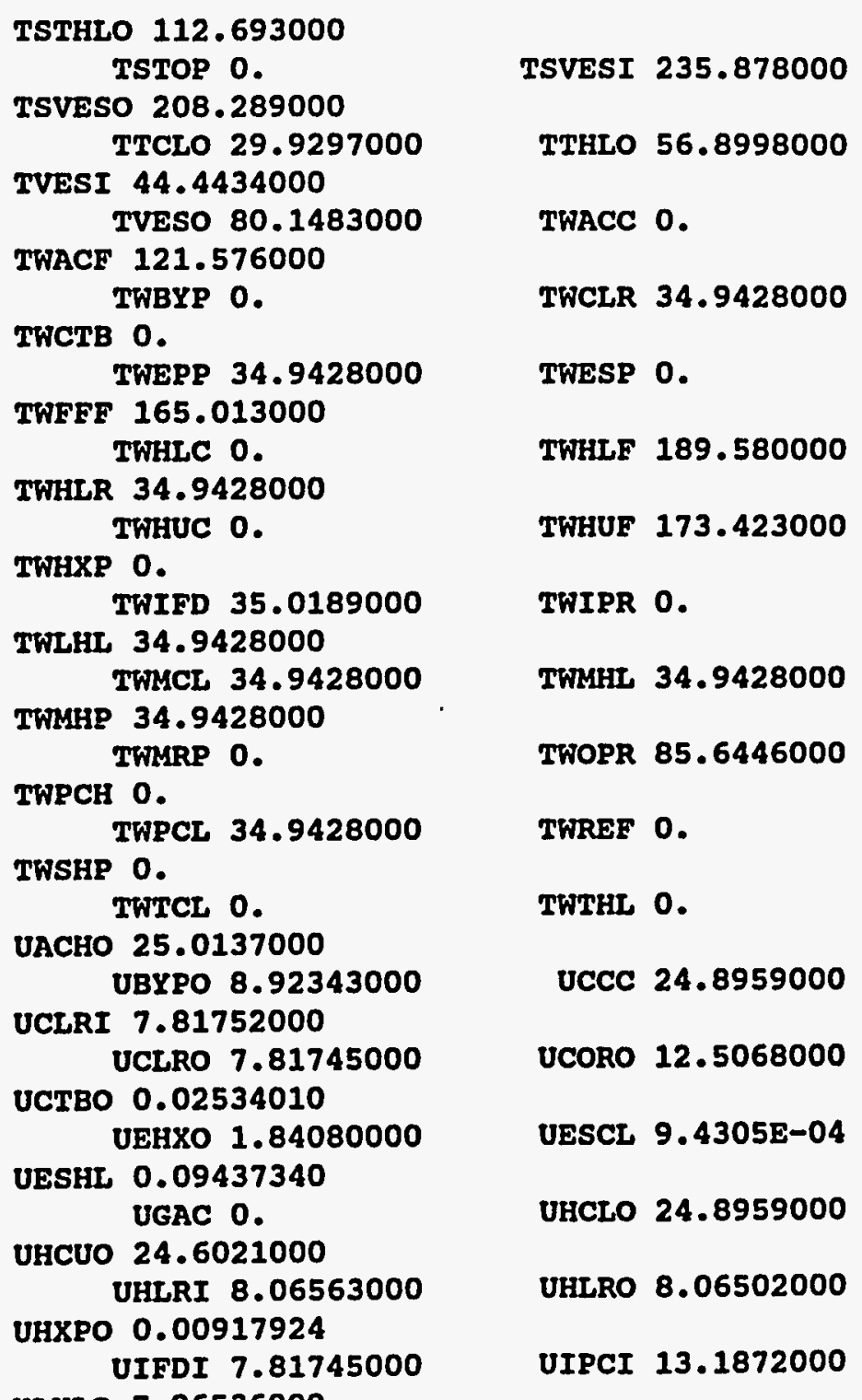

UMCPI 7.81555000

UMHLL 7.91112000 UMHLO 8.06468000

UMHXO 0.78053300 UMRPO 0.00533074

UREFO 0.18417500

USCLX 3.85871000

UTCLO 1.83624000

UTHLO 1.85789000

UVESO 8.06563000 VCICR 0 .

VGGAC 1.55400000 VIRCON 0.

WACHO 1790.16000 พBYPI 353.744000

WCILC 0 .

WCLRI 2143.93000

WCOLC 0 .

WCORO 1790.16000

ผCTBO 2799.77000 WEHXO 2144.00000

WESHL 104.000000 WHCLI 1547.26000

WHCUI 1543.07000 WHCUO 1543.07000

WHLRO 2143.90000 WHXPI 101.284000

WIFDI 2143.93000 พIPCI 2143.90000

WLDSI 14.6000000 WLHLO 2143.90000

WMCPO 2144.00000 WMHLA 2143.90000 WMHLO 2143.90000 WMHXI 2143.90000 WMRPI 58.7873000 WMRPO 58.7875000
UMCPO 7.81784000

UMHXI 7.96536000

UPCHO 0.00697055

USHLX 2.33080000

UVESI 21.0200000

VCOCR 0 .

WACHI 1790.16000

พBYPO 353.744000

WCLRO 2143.93000

WCTBI 2799.77000

WESCL 104.000000

พHCLO 1547.28000

WHLRI 2143.90000

WHXPO 101.229000

WLDCON 14.6000000

WMCLI 2143.93000

WMHLL 2129.30000

WMHXO 2143.97000

WMUSO 14.6000000 
WPCHI 76.8711000 พРСНО 76.8714000 WREFO 200.000000

พSCISC 4

WSDET 2799.77000 WSHLX 2800.00000

WTCLO 2799.77000 WVESI 2143.93000

XCRACH 98.1025000 XCRHCL 43.7258000

XIRACH 98.1025000 XIRHCL 47.9086000

XPRACH 98.1025000 XPRHCL 47.9086000

XSRACH 98.1025000 XSRHCL 47.9086000

299829-5 . 76493000

$299830 \quad 0$

299832

299833

2998351.00000000

$\mathrm{z} 99836 \quad 17$

299846

299847-1.24000000

299849

$299850 \quad 11$

Z99852

$299854 \quad 12$

Z99856-0.80000000

$299857 \quad 0$

299859

299860 F

29986258.8600000

$299863 \quad 21$

299865-0.75000000

$299866 \quad 0$

299868
WREFI 200.000000

พSCLX 2799.77000

WTCLI 2799.77000

FVESO 2143.90000

XCRHCU 68.8227000

XIRHCU 68.8227000

XPRHCU 68.8227000

XSRHCU 68.8227000

$299831 \quad 12$

$299834 \quad 15$

2998371.00000000

299848-2.2000E+08

$299851 \quad F$

2998550.

$\mathrm{z99858} \quad 10$

$299861 \quad 5$

2998640.98000000

299867
$299869 \quad F$

29987158.8600000 Z99874

299876-1.0000E+10 299877

299879

299880

299883

Z99884

$299886 \quad F$

z99887 1.000OE+10 299889

Z99890

$\mathrm{F}$

Z99892-339605.000

299893

299895

299896

$z 99898$

299899

299901

299902-1972 .48000

Z99904

z99905

3

299907-0.20000000

$$
299908
$$

Z99910

z9991

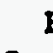

299913

$299914 \quad 1$

299916

299917

12

299919

2999200 .

2999220.

$299923 \quad 21$

299925

2999260.98000000
$299870 \quad 5$

$\mathrm{z} 998751.00000000$

299878

8

z99882-1.0000E+10

$299885 \quad F$

Z99888

Z99891 F

$299894 \quad 5$

299897-10.5161000

Z99900 F

Z99903

$299906 \quad$ F

299909

Z99912-6.9963E-05

$299915 \quad$ F

2999180.

z99921

12

Z99924 0.98000000

$\mathbf{z 9 9 9 7 9}$

10 
Z99980-1.24000000 $\begin{array}{ll}299995 & 12\end{array}$

ZAEEHX 500.000000 ZAEMHX 8750.00000

ZAUHLF $3.3488 E+06$ ZAUHUE $3.3177 E+06$

ZB2BYP 0.24473000 ZB2CLR 1.00000000

ZB2CTB 0.01380000 ZB2EPP 0.42400000

ZB2HLC 0.40518200 ZB2HLR 1.00000000

2B2HXP 1.00000000 ZB2IFD 0.37190600

ZB2LHL 0.98774300 ZB2MCL 1.00000000

ZB2MHP 0.09986400 ZB2MRP 1.00000000

ZB2PCH 1.00000000 ZB2PCL 0.23552800

ZB2SHP 0.59700000 ZB2TCL 0.01380000

ZBDCIL 0 . zBDCOr 0 .

zCVCIL 0 . zevcor 0 .

ZDHACC 150.256000 ZDHBYP 14.2309000

2DHCTB-1.2745E-06 ZDHEPP 23.7187000

ZDHHLC 2699.44000 ZDHHLR-0.46265300

ZDHHXP-0.71966300 ZDHIFD 0 .

ZDHLHL-0. 43457300 ZDHMCL -0.40749600

ZDHMHP 25.1418000
2999960.

ZAUACF $2.5306 E+06$

ZB2ACC 0.45020300

ZB2COR 0.54038900

ZB2ESP 0.01000000

ZB2HUC 0.40518200

ZB2IPR 0.62736500

QB2MHL 1.00000000

ZB2OPR 0.50000000

ZB2REF 1.00000000

ZB2THL 0.79710100

ZBTCNT 0.00774000

ZDECPG 0.05754400

ZDHCLR-0.50672300

ZDHESP 0.00466240

ZDHAUC 238.107000

ZDHIPR-608.837000

ZDHMHL-0. 25996300
ZDHMRP 0.00579261

ZDHPCK 0.01194720

ZDHPCL-0.00150202

ZDHSHP 61.9591000 ZDHTCL 0 .

ZDJEHX 7.6076E-05 ZDJMHX 3.2418E-05

ZDNCON-8.6018E-04 ZDPCON $1.5480 \mathrm{~s}+06$

ZDTCON 0.48389100 ZDWCCS-0.24569400

ZEPPOI 3.0850E+22 ZESPOI $5.7700 E+21$

ZFAACF 53.0709000 ZFAHLF 53.0709000 ZFEGAC 0

ZFEMUS 0.00681001

ZFPOI $4.6652 \mathrm{E}+18$ ZFRCNT 0 .

ZHOANS 187933.000 ZKOMCP 2798.51000

ZHFHLF 178291.000 ZHFHUF 173687.000

ZHLHLF 2212.85000 ZHLHUF 2216.67000

ZHPMCP 0.99990000 ZHPMHX 6802.45000

ZHSMHX 10493.1000 ZHTACF 159027.000

ZHTHUE 173687.000 ZIACOR 1074.10000

ZIICIL $3.4001 \mathrm{E}+06$ ZIICOL $1.8414 \mathrm{E}+06$

ZIPACC $2.9267 E+06$ ZIPBYP 3.1580E+06

ZIPCTB 150051.000 ZIPEPP $1.6688 \mathrm{E}+06$
ZDHOPR 0 .

ZDHREF-1.5506E-05

ZDHTHL 0 .

ZDLCON-0.01537870

ZDRCNT 1.00000000

ZEIPOI $1.8931 \mathrm{E}+22$

ZEXPOI $4.4457 \mathrm{E}+20$

ZFAHUF 53.0709000

ZFEPOI $4.6652 E+18$

ZFSCON 1.00000000

ZHFACF 159027.000

ZHLACF 1980.77000

ZHPEHX 10101.1000

ZHSEHX 207.757000

ZHTHLF 178291.000

ZIBCOR 1094.48000

ZIJSCC-3.1390E+08

ZIPCLR $3.2675 E+06$

ZIPESP 149995.000 
ZIPGAC 1.6904E+06

ZIPHLC 2.9253E+06

ZIPHUC 2.8828E+06

ZIPHXP 150000.000

ZIPIPR 3.2574E+06

ZIPLHL $1.6879 \mathrm{E}+06$

ZIPMHL $1.6983 \mathrm{E}+06$

ZIPMHP 1.6968E+06

ZIPOPR 1.8763E+06

ZIPPCH 150000.000

ZIPREF 1000000.00

ZIPSHP 266012.000

ZIPTHL 182725.000

ZIRREF 1085.92000

ZITLDS 0 .

ZITMUS 0 .

ZIWCOL 2144.00000 ZJDACF 0 .

ZJDHLF 0 . ZJDHUF 0.

ZJFHLF $5.5324 \mathrm{E}+08$ ZJFHUF 4.8490E+08

ZJPMCP 1.00000000 ZJWACF 3.0301E+08

2JWHUF $4.8489 \mathrm{E}+08$ ZKCACC 0.04398380

ZKCBYP 0.06042160 ZKCCLR 0 .

ZKCCTB 0.97259000 ZKCEPP 0.28800000

ZKCHLC 0.04758540 ZKCHLF 1.1748E-05

ZKCHUC 0.04758540 ZKCHUF $1.1748 \mathrm{E}-05$

ZKCIFD 0.09421410 ZKCIPR 0.03193710 ZKCMCL 0.
ZIPHLR $1.8416 \mathrm{E}+06$

ZIPIFD 3.1879E+06

ZIPMCL 3.2931E+06

ZIPMRP 150000.000

ZIPPCL $1.6178 \mathrm{E}+06$

2IPTCL 230175.000

ZITGAC 39.6870000

ZIFCIL 2144.00000

ZJDCPG 1.8990E+07

ZJFACF 3.0301E+08

ZJNCPG $3.1101 E+08$

ZJWHLF $5.5330 \mathrm{E}+08$

ZKCACF 1.6192E-05

ZKCCOR 0.21124200

ZKCESP 0.49500000

ZKCHLR 0.

2KCHXP 0.

ZKCLHL $1.5023 E-04$
ZKCMHL 0.

ZKCMRP 0 .

ZKCOPR 0.25000000

ZKCPCL 0.38223600 ZKCREF 0 .

ZKCTCL 0 . ZKCTHL 0.04116780

ZKFHLF 1.0239E-05 ZKFHUF 1.0239E-05

ZKGBYP 5.16987000 ZKGCLR-133.122000 ZKGEPP 0 .

ZKGESP 29.4300000

ZKGHLR 122.625000 2KGHUC 5.16987000

ZKGIFD 0 .

ZKGIPR 29.4300000

ZKGMCL 0 . ZKGMHL 0 .

ZKGMRP 0 .

ZKGOPR 41.7219000

ZKGPCL-47.8728000 ZKGREF 0 .

ZKGTCL-29.4300000 2KGTHL 29.4300000

ZLAGAC 20.0272000 ZLHCL 0.98102500

ZNOMCP 1.00000000 ZNPCCS 2.00000000

ZNWMHX 6.00000000 zOCCIL 0.60000000

ZPOANS $3.1180 \mathrm{E}+06$ ZPOGAC 1.5864E+06 ZPACF $1.7083 \mathrm{E}+06$

2PCACH (50) $5.5555 E+33$ ZPCHCL (50) $5.5555 E+33$ ZPCHCU (50) $5.5555 E+33$ ZPDACC 336023.000
ZKCMHP 0.81024500

2KCPCH 0 .

ZKCSHP 0.16240900

ZKFACF $1.4683 \mathrm{E}-05$

ZKGACC 5.16987000

2KGCTB 0.

ZKGHLC 5.16987000

2KGHXP 0 .

ZKGLHL-17.9523000

ZKGMHP 0 .

2KGPCH 0 .

ZKGSHP 0.

ZLACH 0.98102500

ZLHCU 0.98102500

ZNWEHX 35.0000000

zOCCOL 0.60000000

ZPOMCP $1.6418 \mathrm{E}+06$

ZPDBYP 43575.6000 
ZPDCLR 33565.8000 ZPDCTB 0.35473300 ZPDESP 4.90742000 ZPDHLC 321186.000 ZPDHUC 316531.000 ZPDHXP 0.04646020

ZPDIPR 95514.0000 ZPDLHL 34200.4000

ZPDMHL 35056.5000 ZPDMHP 334.688000

ZPDOPR 84005.9000 ZPDPCH 0.02679180

ZPDREF 18.4175000 ZPDSHP 2966.48000

ZPDTHL 1884.81000 ZPFACC $1.2129 \mathrm{E}+06$

ZPFCLR 13638.4000 ZPFCTB 0.

ZPFESP 20.9974000 ZPFGAC 0.

ZPFHLR 11169.7000 ZPFHUंC 1.2166E+06 ZPFIFD 69832.9000 ZPFIPR 26018.8000 ZPFMCL 25568.6000 ZPFMHL 11184.0000 ZPFMRP 0 .

ZPFOPR 21074.1000

ZPFPCL 18791.9000 ZPFREF 0 .

ZPFTCL 602.010000 ZPFTHL 585.577000 ZPGBYP 5658.33000 ZPGCLR-146233.000 ZPGEPP 0 .

ZPGESP 32432.0000 ZPGHLC 5358.07000
ZPDEPP 1861.63000

ZPDHLR 35058.0000

ZPDIFD 242678.000

ZPDMCL 33566.1000

ZPDMRP 0.01566900

ZPDPCL 33558.7000

ZPDTCL 1862.70000

ZPFBYP 5396.20000

ZPFEPP 18623.0000

ZPEHLC $1.2123 E+06$

ZPFHXP 0

ZPFLHL 16654.1000

ZPFMHP 26378.7000

ZPFPCH 0 .

ZPFSHP 84246.8000

ZPGACC 5552.95000

ZPGCTB 0.

ZPGGAC 103984.000
ZPGHLR 132186.000

ZPGHXP 0 .

ZPGIFD 0

ZPGLHL-19354.0000 ZPGMCL 0.

ZPGMHP 0 .

ZPGMRP 0.

ZPGPCH 0 . ZPGPCL-52602 . 3000

ZPGSHP 0.

ZPGTCL-32516.6000

ZPHLF 1.7077E+06 ZPHUF $1.6608 \mathrm{E}+06$

ZPIBYP 0 . ZPICLR 0.

ZPIEPP 0 . ZPIESP 0.

ZPIHLR 0. ZPIHUC 0 .

ZPIIFD 0 . ZPIIPR 0 .

ZPIMCL 0 . ZPIMHL 0 . ZPIMRP 0. ZPIOPR 0 .

ZPIPCL 0. ZPIREF 0 .

ZPITCL 0 . ZPITHL 0 . ZPLCOL $5.5555 E+33$ ZPPSCC 81985.7000 ZPSACH(50) 5.5555E+33 ZPSHCL (50) $5.5555 E+33$ ZPSHCU (50) $5.5555 E+33$ ZPTMCP 1.00000000

ZQCACH (50) $5.5555 E+33$ ZQCHCL (50) $5.5555 E+33$ ZQCHCU (50) $5.5555 \mathrm{E}+33$ ZQSACH (5O) 5.5555E+33 ZQSHCL(50) $5.5555 E+33$
ZPGIPR 32328.1000

ZPGMHL 0.

ZPGOPR 44813.5000

ZPGREF 0 .

2PGTHL 32140.3000

ZPIACC 0.

ZPICTB 0.

ZPIHLC 0.

ZPIHXP 0 .

ZPILHL 0 .

ZPIMHP 0 .

ZPIPCH 0 .

ZPISHP 0.

ZPLCIL $5.5555 \mathrm{E}+33$

ZPRMCS $3.2931 E+06$ 


\begin{tabular}{|c|c|c|c|}
\hline 2QSHCU (50) & $5.5555 \mathrm{E}+33$ & & \\
\hline ZQWACH (50) & $5.5555 \mathrm{E}+33$ & ZQWHCL (50) & $5.5555 \mathrm{E}+33$ \\
\hline \multicolumn{4}{|c|}{ ZQWHCU(50) 5} \\
\hline ZROCNT & -3.56703000 & ZROICR & 0.98000000 \\
\hline \multicolumn{4}{|c|}{ ZROOCR 0} \\
\hline ZRACOR & 0 & ZRBCOR & 0. \\
\hline \multicolumn{4}{|c|}{ ZRCACF 80859.8000} \\
\hline ZRCHLE & 80859.8000 & ZRCHUE & 80859.8000 \\
\hline ZRFCOR 0 & & & \\
\hline ZRMPOI & -3.56703000 & ZSCNT & 0 \\
\hline \multicolumn{4}{|c|}{ ZSMACH 1.00000000} \\
\hline ZSMHCL & 1.70348000 & ZSMHCU & 1.48739000 \\
\hline \multicolumn{4}{|c|}{ ZTOACF 121.629000} \\
\hline \multicolumn{4}{|c|}{ ZTACC 85.6446000} \\
\hline ZTBYP & 52.4385000 & ZTCACH(50) & $5.5555 \mathrm{E}+33$ \\
\hline \multicolumn{4}{|c|}{ ZTCEHX 8.88197000 } \\
\hline ZTCHCL (50) & $5.5555 E+33$ & $\operatorname{2TCHCU}(50)$ & $5.5555 E+33$ \\
\hline \multicolumn{4}{|c|}{ ZTCLR 44.4433000} \\
\hline ZTCMHX & 14.0289000 & ZTCTB & 29.9297000 \\
\hline \multicolumn{4}{|c|}{ ZTDCDH 0} \\
\hline ZTEPP & 43.8247000 & ZTESP & 36.7836000 \\
\hline \multicolumn{4}{|c|}{ ZTFCOR 205.381000 } \\
\hline ZTHEHX & 7.17500000 & ZTHLC & 131.141000 \\
\hline \multicolumn{4}{|c|}{ ZTHLR 80.0268000} \\
\hline ZTHMHX & 22.9649000 & ZTHUC & 120.837000 \\
\hline \multicolumn{4}{|c|}{ ZTHXP 34.9428000} \\
\hline ZTIFD & 44.4434000 & ZTIPR & 44.4794000 \\
\hline \multicolumn{4}{|c|}{ ZTLHL 79.8645000} \\
\hline ZTMCL & 44.4626000 & 2TMEHX & 7.99815000 \\
\hline \multicolumn{4}{|c|}{ ZTMHL 79.9591000} \\
\hline ZTMHP & 43.9584000 & ZTMMHX & 18.1314000 \\
\hline \multicolumn{4}{|c|}{ ZTMRP 34.9428000} \\
\hline ZTOPR & 85.6446000 & ZTPCH & 34.9428000 \\
\hline \multicolumn{4}{|c|}{$\begin{array}{l}\text { ZTPCL } 43.8101000 \\
\text { ZTREF } 67.5966000\end{array}$} \\
\hline ZTRE & תחת & & 8000 \\
\hline
\end{tabular}

ZTSMCP $5.5555 \mathrm{E}+33$ ZTTHL 56.8998000

ZTWACH (50) 5.5555E+33 ZTWHCL(50) $5.5555 E+33$ ZTWHCU (50) $5.5555 E+33$

ZTXACH (50) 5.5555E+33 2TXHCL(50) $5.5555 E+33$ ZTXHCU (50) 5.5555E+33 ZUEEHX 100014.000 ZWOANS 2144.00000

ZWCCON 2144.00000 ZWLCOR 0.86414400 ZWPMCP 1.00000000

ZWUCOR $0.84857100 \quad$ ZZSEED 55555555 2ZWEHX 104.000000 Z2WMHX 2800.00000
ZUEMHX 1.7420E+07

zzzISC 0. 
. 
Internal Distribution

1. R. B. Battle

2. J. E. Cleaves

3. D. N. Fry

4. R. M. Harrington

5-7. J. A. March-Leuba

8. D. W. McDonald

9. G. R. McNutt

10. D. L. Moses

11. D. L. Selby
12. C. D. West

13. J. D. White

14. G. L. Yoder

15-16. Central Research Library

17. Document Reference Section

18. ORNL Patent Section

19-20. Laboratory Records Department

21. Laboratory Records Department, RC

22-31. Y-12 Technical Library

\section{External Distribution}

32. U.S. Department of Energy, Oak Ridge Operations, FEDC, MS-8218, P.O. Box 2009, Oak Ridge, TN 37831-8218

33-34. Office of Scientific and Technical Information, P.O. Box 62, Oak Ridge, TN 37831 


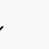

